\title{
Microcystin Toxins at Potentially Hazardous Levels in Algal Dietary Supplements \\ Revealed by a Combination of Bioassay, Immunoassay and Mass Spectrometric Methods
}

Todd R. Miller ${ }^{\mathrm{a}}$, Ame Xiong ${ }^{\mathrm{a}}$, Jonathan R. Deeds ${ }^{\mathrm{b}}$, Ingunn A. Samdal ${ }^{\mathrm{c}}$, Christopher O. Miles ${ }^{\mathrm{d}}$

Affiliations

aJoseph J. Zilber School of Public Health, University of Wisconsin - Milwaukee, Milwaukee,

WI, USA

bUnited States Food and Drug Administration, Center for Food Safety and Applied Nutrition,

College Park, MD,USA

'Norwegian Veterinary Institute, P. O. Box 750 Sentrum, N-0106, Oslo, Norway

dMeasurement Science and Standards, National Research Council, 1411 Oxford Street, Halifax,

NS, B3H 3Z1, Canada

\section{Supplemental Material*}

Table of Contents

Figure S1

Figure S2

Figure S3

Figure S4

Figure S5

Figure S6

Figure S7

Table S1

Table S2

Table S3

Figure S8

Figure S9

Figure S10

Figure S1 1

Figure S12
MS/MS spectra of MC-LR, -LA, -YR, [Dha $\left.{ }^{7}\right] \mathrm{MC}-\mathrm{LR}$ and NOD-R

MS/MS spectra of MC-WR, -LW, -HtyR, -HilR, -LF, and -LY MS/MS spectra of Apt-B, Apt-F, Cpt-1020, Cpt-1041, Cpt-1007 and Mgn-690S6

Chromatograms of MC-LR, -LA, -YR, [Dha $]$ MC-LR and NOD-R

Chromatograms of MC-WR, -LW, -HtyR, -HilR, -LF, and -LY

Chromatograms of Apt-B \& -F, Cpt-1020, -1041, \& -1007 and Mgn-690

MRM chromatograms of MC-LR and MC-(H4)YR in supplement TM07

Compound-specific LC-MS/MS parameters

Non-compound-specific LC-MS/MS parameters

Recoveries of target analytes after extraction and analysis by LC-MS/MS

LC-HRMS chromatograms of MC-LR (1) and its congeners

LC-HRMS chromatograms of MC-RR (6) and its congeners

LC-HRMS chromatograms of MC-YR (11) and its congeners

LC-HRMS chromatograms of MC-LA (16) and its congeners

LC-HRMS chromatograms of MC-(H4)YR (21) and its congeners
Page

S4

$\mathrm{S} 5$

$\mathrm{S} 7$

S8

S9

S10

S 11

S12

S13

S14

S15

S16

$\mathrm{S} 17$

S18 
Table of Contents (cont.)

Figure S13 LC-HRMS chromatograms of MC-YR, -(H2)YR -(H4)YR, and -Y(OMe)R

Figure S14

LC-HRMS chromatograms of $\mathbf{2 7 - 3 0}$ and $\mathbf{3 2}$

Figure S15

LC-HRMS chromatograms of 5, 27, 33 and 34

S19

Figure S16

LC-HRMS chromatograms of MC-(H4)Y(H4)Y (43)

Figure S17

HRMS of MC-(H4) $\mathrm{Y}(\mathrm{H} 4) \mathrm{Y}(\mathbf{4 3})$

S21

Figure S18

HRMS/MS spectra of $16,43-\mathrm{H}_{2} \mathrm{O}$ and 43

S22

$\mathrm{S} 23$

Figure S19

Expansion of the HRMS/MS spectra of 16, 43- $\mathrm{H} 2 \mathrm{O}$ and $\mathbf{4 3}$

S24

S25

Figure S20

Expansion of the HRMS/MS spectra of 16, 43- $\mathrm{H} 2 \mathrm{O}$ and $\mathbf{4 3}$

S26

Figure S21

Expansion of the HRMS/MS spectra of 16, 43- $\mathrm{H} 2 \mathrm{O}$ and 43

S27

Figure S22

Expansion of the HRMS/MS spectra of 16, 43- $\mathrm{H} 2 \mathrm{O}$ and $\mathbf{4 3}$

S28

Figure S23

Expansion of the HRMS/MS spectra of 16, 43- $\mathrm{H} 2 \mathrm{O}$ and $\mathbf{4 3}$

S29

Figure S24

LC-HRMS chromatograms of MC-LAbu (41)

S30

Figure S25

HRMS/MS spectra of MC-LAbu (41) and isobaric microcystins

Figure S26

LC-HRMS chromatograms of MC-LV (42)

S31

Figure S27

HRMS/MS spectra of MC-LV (42) and isobaric microcystins

S 32

S33

Figure S28

HRMS/MS spectra of MC-LA (16), MC-LAbu (41) and MC-LV (42)

S34

Figure S29

Expansion of the HRMS/MS spectra of 16, 41 and $\mathbf{4 2}$

S35

Figure S30

Expansion of the HRMS/MS spectra of 16, 41 and $\mathbf{4 2}$

S36

Figure S31

Figure S32

Expansion of the HRMS/MS spectra of 16, 41 and $\mathbf{4 2}$

S37

LC-HRMS/MS chromatograms and HRMS/MS spectra of $\mathbf{1 6} \quad$ S38

Figure S33

HRMS/MS spectra of $\mathbf{1 6}$ and isobaric microcystins

S39

LC-HRMS/MS chromatograms of MC-LY (35) $\quad$ S40

Figure S34

Figure S35

HRMS/MS spectra of $\mathbf{3 5}$ and isobaric microcystins

$\mathrm{S} 41$

Figure S36

Figure S37

LC-HRMS/MS chromatograms of MC-LF (36)

$\mathrm{S} 42$

HRMS/MS spectra of $\mathbf{3 6}$ and isobaric microcystins

Figure S38

Figure S39

LC-HRMS/MS chromatograms of [D-Ser']MC-LA (44)

$\mathrm{S} 43$

S44

HRMS/MS spectra of $\mathbf{4 4}$ and isobaric microcystins

S45

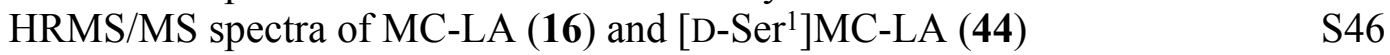

Figure S40

Expansion of the HRMS/MS spectra of $\mathbf{1 6}$ and $\mathbf{4 4}$

S47

Expansion of the HRMS/MS spectra of $\mathbf{1 6}$ and $\mathbf{4 4}$

S48

Figure S42

Figure S43

Figure S44

Figure S45

Figure S46

Figure S47

Figure S48

Figure S49

Expansion of the HRMS/MS spectra of $\mathbf{1 6}$ and $\mathbf{4 4}$

S49

Expansion of the HRMS/MS spectra of $\mathbf{1 6}$ and $\mathbf{4 4}$

LC-HRMS/MS chromatograms of [D-Ser'] ${ }^{1}$ MC-LR (34)

S50

LC-HRMS/MS chromatograms and HRMS/MS spect of 29 and 32

HRMS/MS spectra of $\mathbf{2 9}$ and $\mathbf{3 2}$ and isobaric microcystins $\quad$ S53

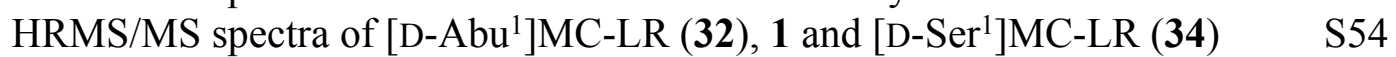

Expansion of the HRMS/MS spectra of 32, 1 and $\mathbf{3 4}$

S55

Expansion of the HRMS/MS spectra of 32, 1 and $\mathbf{3 4}$

S56

Expansion of the HRMS/MS spectra of 32, 1 and 34

S57

Expansion of the HRMS/MS spectra of 32, 1 and $\mathbf{3 4} \quad$ S58

Figure S52

Figure S53

Expansion of the HRMS/MS spectra of 32, 1 and 34

S59

LC-HRMS/MS chromatograms of desmethylMC-LR congeners

S60

Figure $\mathrm{S} 54$

HRMS/MS spectra of desmethylMC-LR congeners 2-4, 37 and 38

S61

Figure S56

LC-HRMS/MS chromatograms of desmethylMC-(H4)YR congeners

S62

Figure S57

HRMS/MS spectra of desmethylMC-YR congeners 22-24, 39 and 40

S63 
Table of Contents (cont.)

Page

Figure S58 LC-HRMS chromatograms of [ $\left.\mathrm{Mser}^{7}\right] \mathrm{MC}$ congeners with thiol derivatization S64

Figure S59 HRMS/MS spectra of MC-LR (1), MC-YR (11), and MC-(H4)YR (21) S65

Figure S60 LC-HRMS/MS chromatograms and HRMS/MS spectra of desmethylMC-LA S66

Figure S61 HRMS/MS spectra of desmethylMC-LA congeners including 17-19 $\quad$ S67

Figure S62 LC-HRMS/MS chromatograms and HRMS/MS spectra of [Mser $\left.{ }^{7}\right]$ MC-LA $\quad$ S68

Figure S63 HRMS/MS spectra of [Mser $\left.{ }^{7}\right] \mathrm{MC}-\mathrm{LA}(\mathbf{2 0})$ and an isobaric congener $\quad$ S69

$\begin{array}{lll}\left.\text { Figure S64 HRMS/MS spectra of [Mser }{ }^{7}\right] \mathrm{MC}-L R(5) & \text { S70 }\end{array}$

Figure S65 LC-HRMS/MS chromatograms and HRMS/MS spectra of desmethylMC-RR S71

Figure S66 HRMS/MS spectra of [Asp $\left.{ }^{3}\right] \mathrm{MC}-\mathrm{RR}(\mathbf{8})$ and [Dha7 $] \mathrm{MC}-\mathrm{RR}(\mathbf{9}) \quad \mathrm{S} 72$

$\begin{array}{lll}\text { Figure S67 } & \text { HRMS/MS spectra of MC-M(O)R (27) and MC-FR (28) } & \text { S73 }\end{array}$

$\begin{array}{lll}\text { Figure S68 } & \mathrm{HRMS} / \mathrm{MS} \text { of }\left[\mathrm{M}+\mathrm{H}-\mathrm{CH}_{4} \mathrm{SO}\right]^{+} \text {for MC-M(O)R (27) } & \mathrm{S} 74\end{array}$

Figure S69 HRMS/MS spectra for desmethylMC-YR congeners 12-14 $\quad$ S75

Figure S70 HRMS/MS spectra of $[\mathrm{M}+2 \mathrm{H}]^{2+}$ and $[\mathrm{M}+\mathrm{H}]^{+}$of MC-RR (6) S76

$\begin{array}{lll}\text { Figure S71 } & \text { HRMS/MS spectrum of MC-(H2)YR (26) } & \text { S77 }\end{array}$

$\begin{array}{lll}\text { Figure S72 } & \text { HRMS/MS spectrum of MC-WR (30) } & \text { S78 }\end{array}$

$\begin{array}{lll}\text { Figure S73 } & \text { HRMS/MS spectrum of MC-Y(OMe)R (31) } & \text { S79 }\end{array}$

Table S4 HRMS/MS $m / z$ shifts for product ions of $\mathbf{1 6}$ and 41-44 $\quad$ S80

Table S5 HRMS/MS $m / z$ shifts for product ions of $\mathbf{1}, \mathbf{3 2}$ and $\mathbf{3 4} \quad$ S81

$\begin{array}{lll} & \text { Links to recall notices for supplements TM07 and TM11 } & \text { S82 } \\ \text { Table S6 } & \text { Estimation of total microcystin recalled } & \text { S83 }\end{array}$

*All LC-HRMS/MS chromatograms and HRMS/MS spectra presented in the Supporting Information were obtained from extracts of supplement sample TM07. 

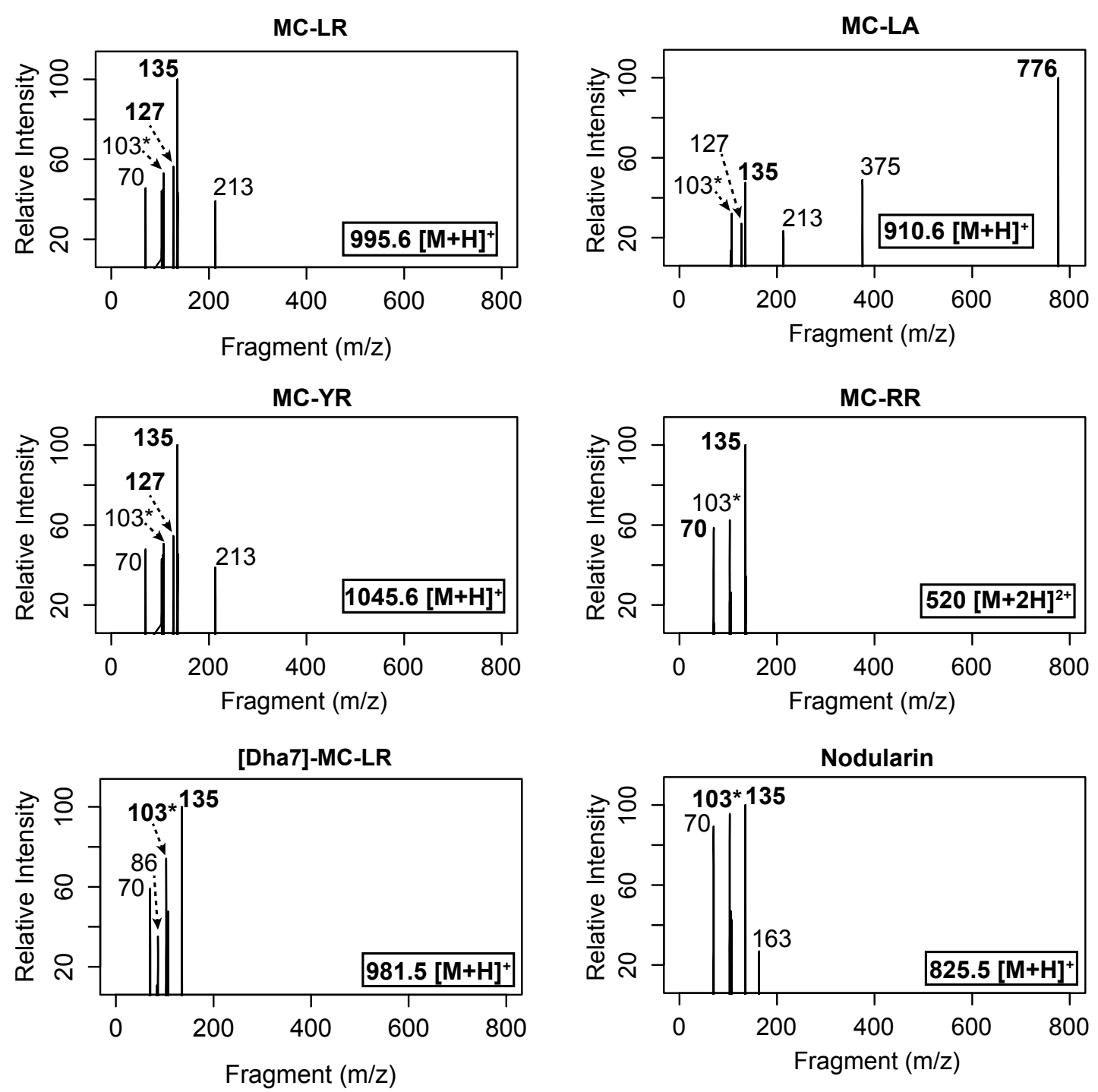

Figure S1. Product ion spectra for standards of microcystins (MC-LR (1), MC-LA (16), MC-RR (6), MC-YR (11), and [Dha $\left.{ }^{7}\right]$ MC-LR (4)) and nodularin-R. The peak near $m / z 103$ for microcystins may be composed of three ions of approximately $m / z 103,105$, and/or 107 . Fix the label for [Dha ${ }^{7}$ MC-LR in the Figure itself! Also, Nodularin-R in the Figure. 

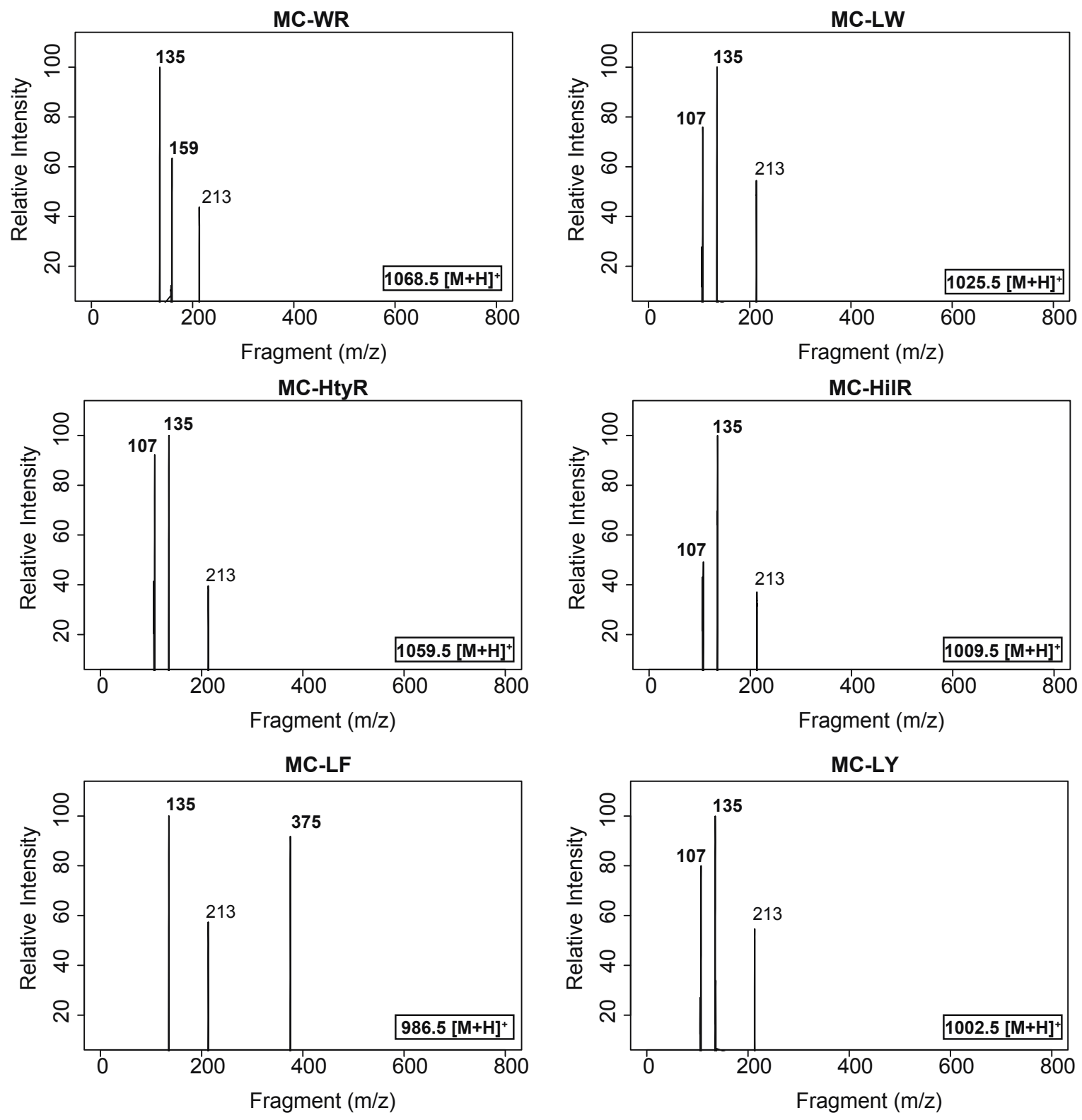

Figure S2. Product ion spectra for standards of microcystins (MC-WR (30), MC-LW, MCHtyR, MC-HilR (29), MC-LF (36), MC-LY (35)). 

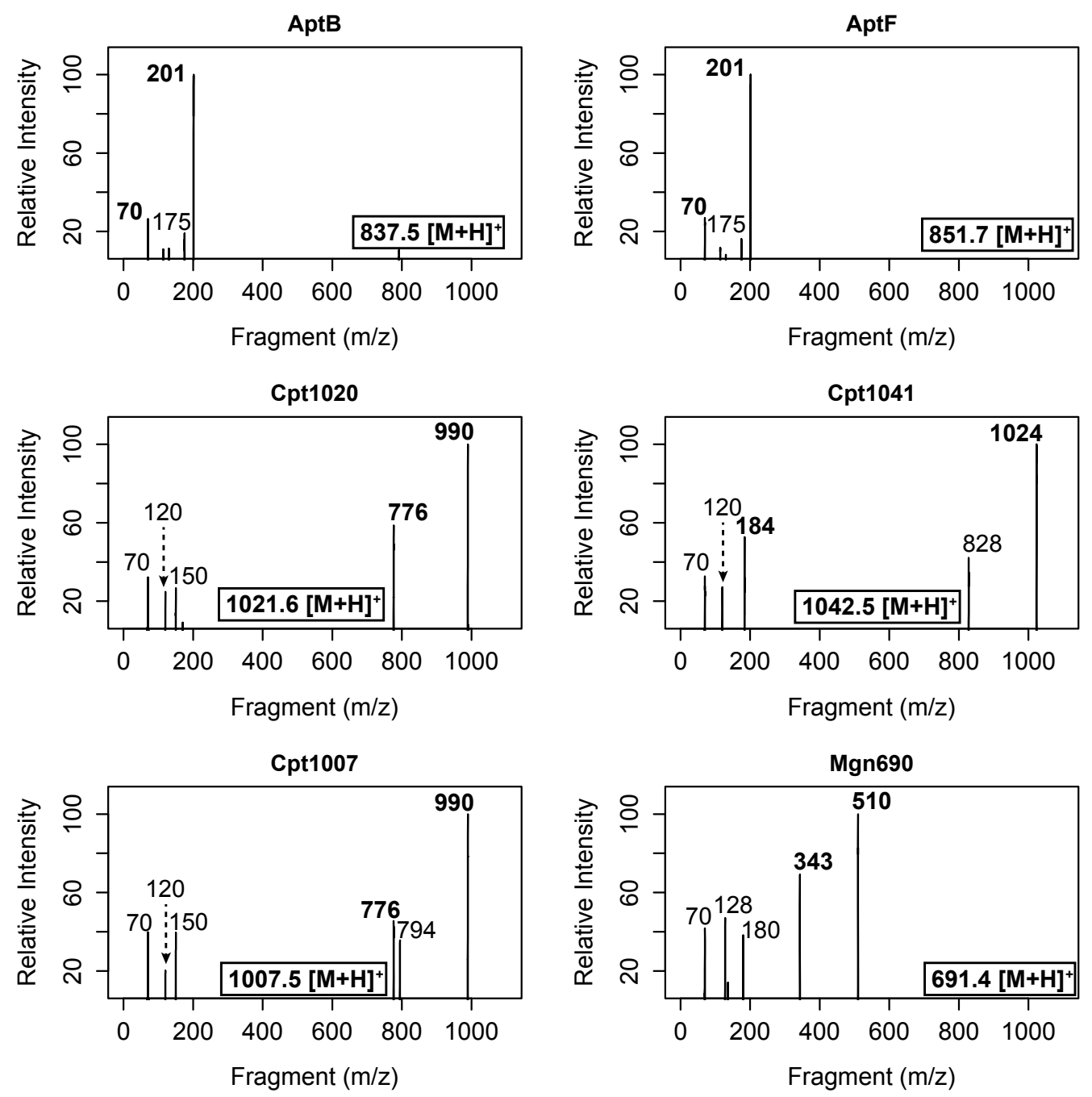

Figure S3. Product ion spectra for anabaenopeptins (Apt), cyanopeptolins (Cpt) and microginin690 (Mgn-690). Insert hyphens into the abbreviated names on the Figures! 
MCLR

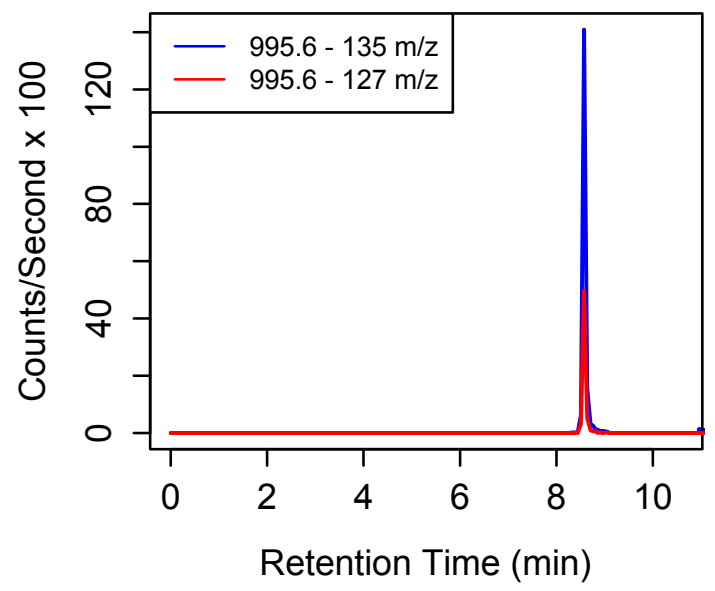

MCRR

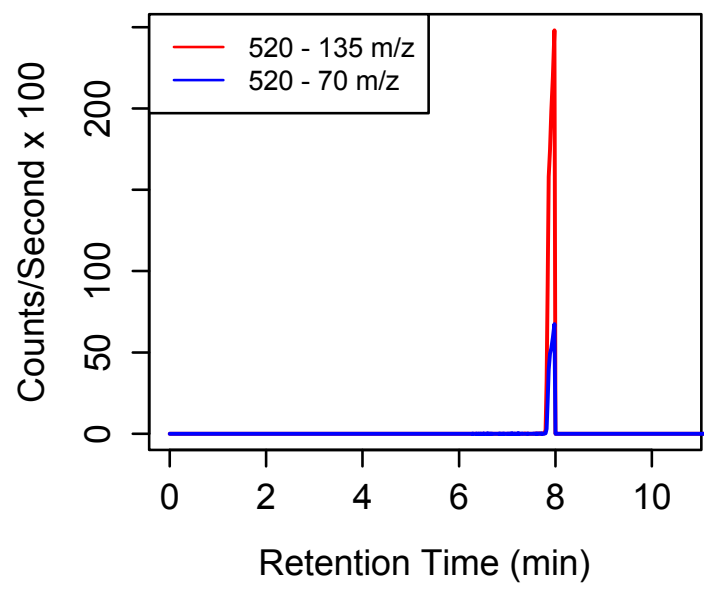

[Dha7]-MC-LR

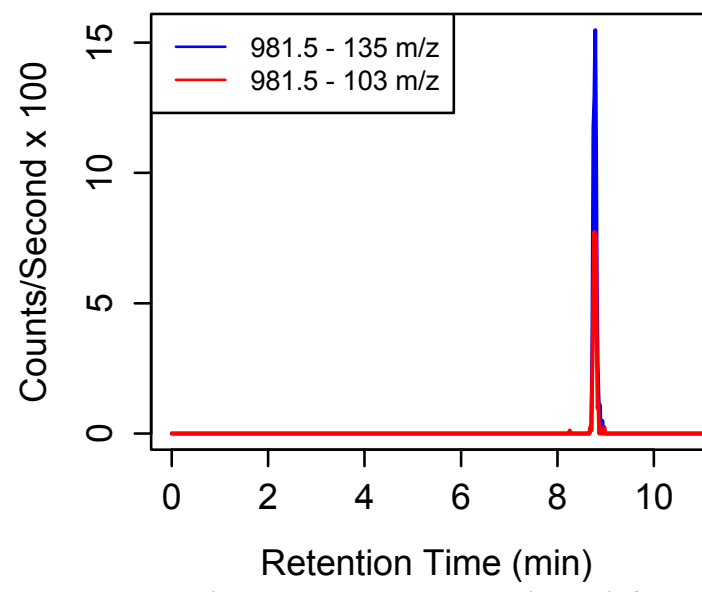

Figure S4. Chromatograms produced from a $50 \mu \mathrm{g} / 1$ standard of microcystins (MC-LR (1), MC-LA (16), MC-RR (6), MC-YR (11), and [Dha ] MC-LR (4) and nodularin-R. Insert hyphens correctly into MC-names. NOD-R. Fprmat [Dha ${ }^{7}$ MC-LR correctly in the Figure label.

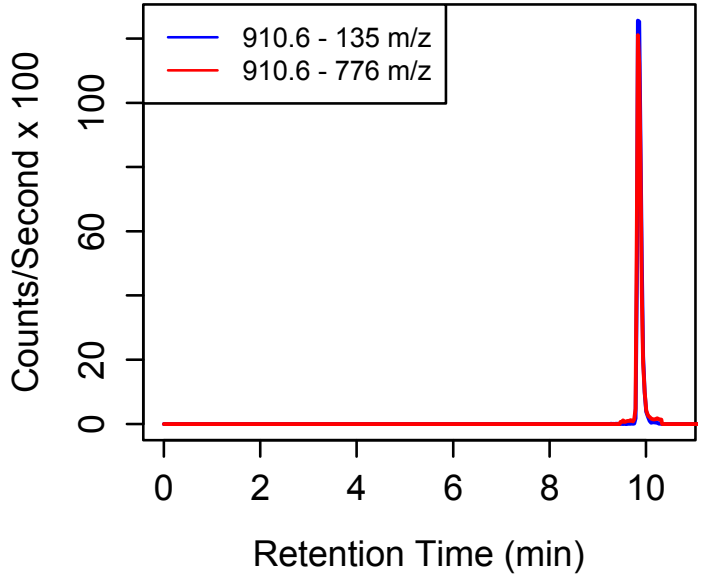

MCYR
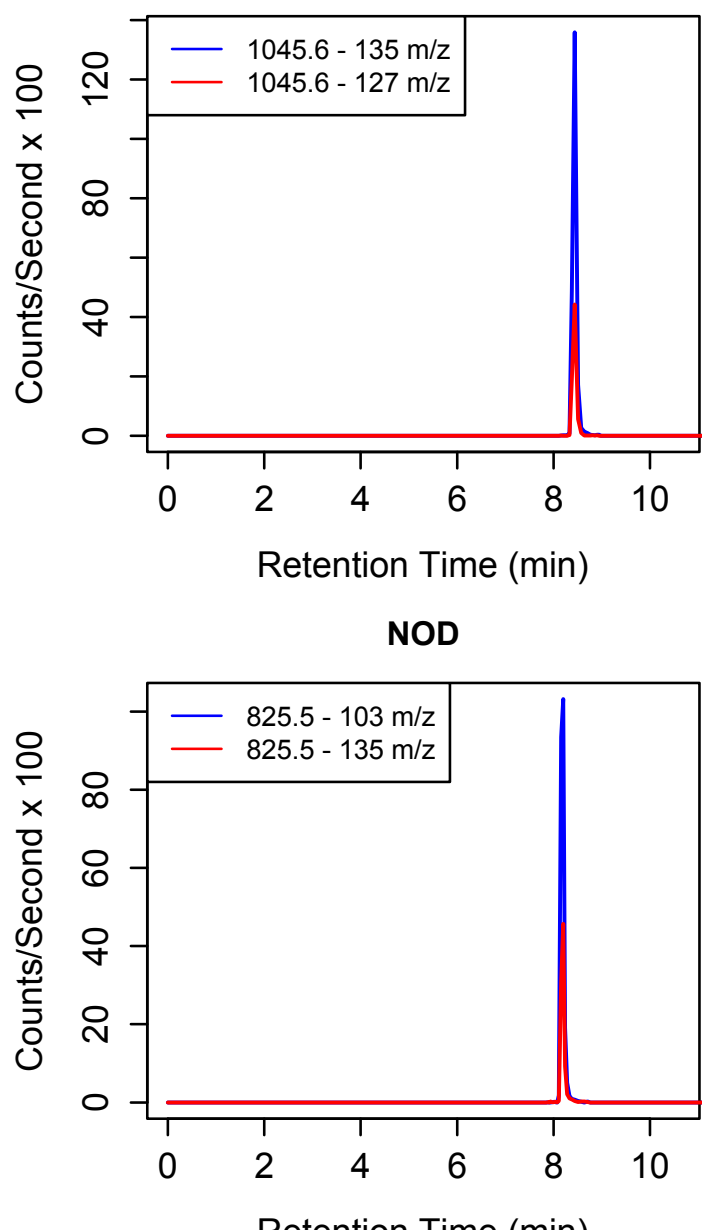

Retention Time (min) 

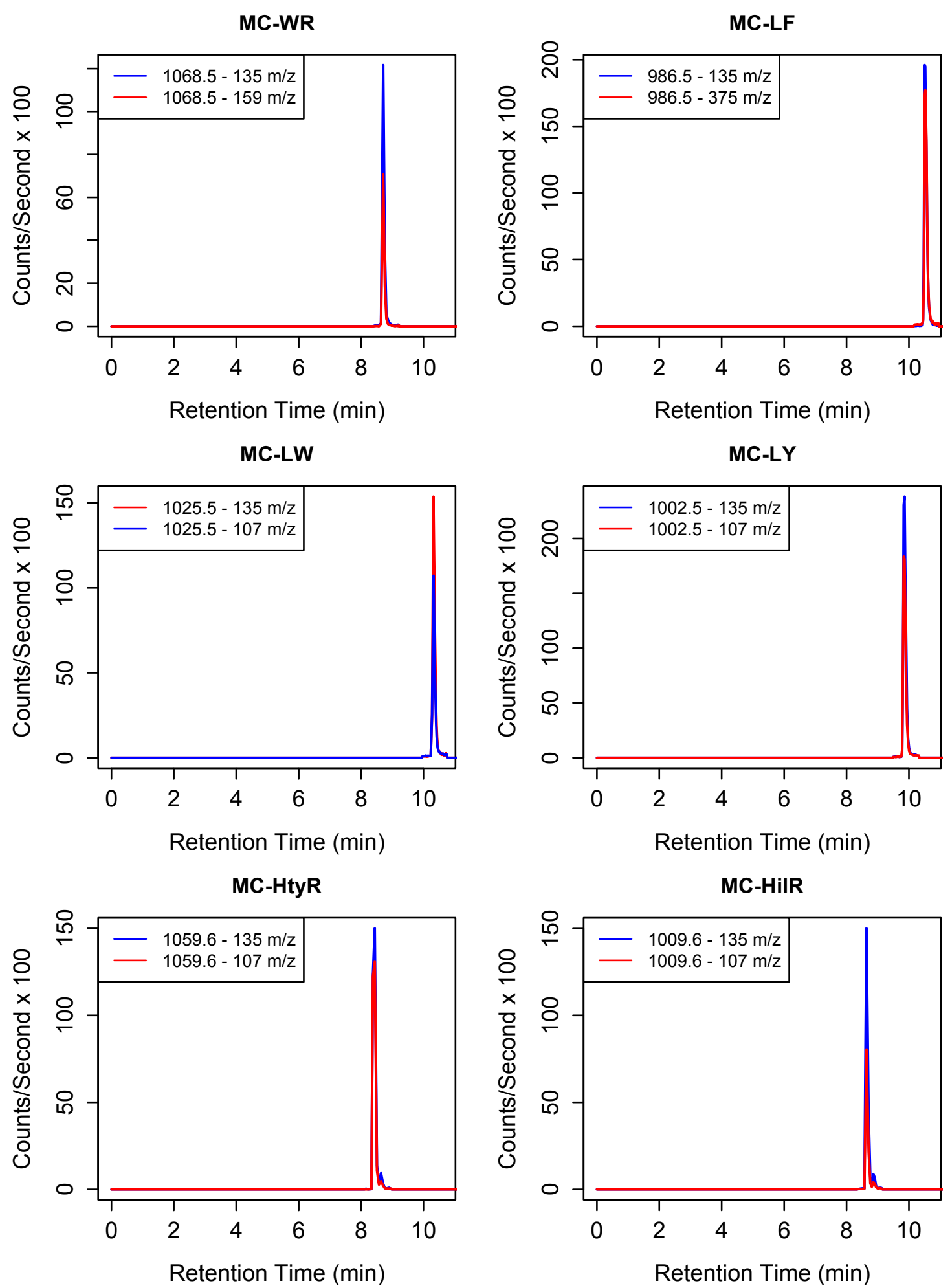

Figure S5. Chromatograms produced from $50 \mu \mathrm{g} / 1$ standards of microcystins MC-WR (30), MC-LF (36), MC-LW, MC-LY (35), MC-HtyR, and MC-HilR (29). 
Cpt1007

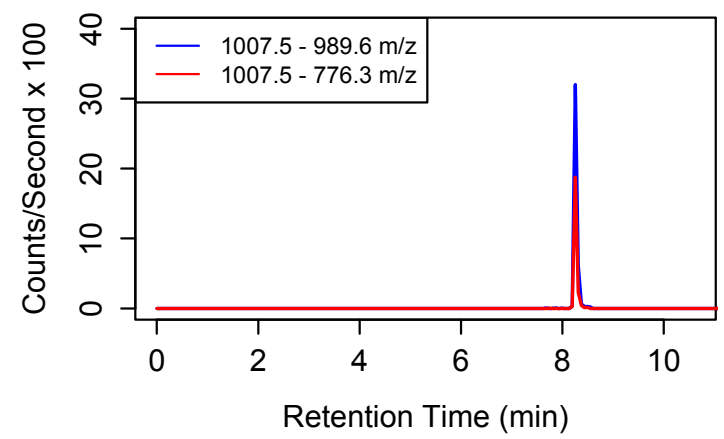

Cpt1020

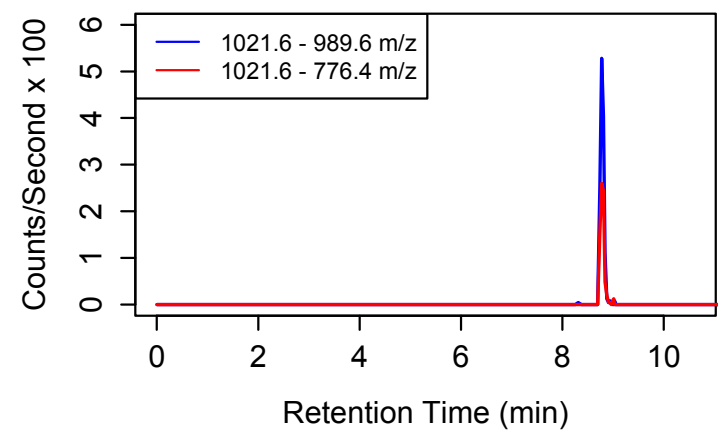

AptF

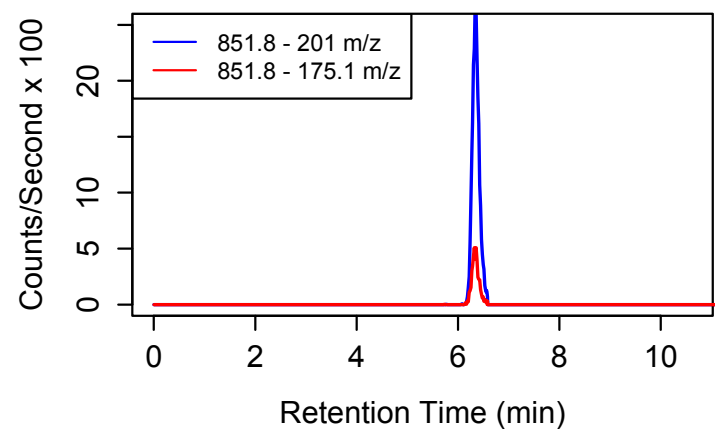

Cpt1041

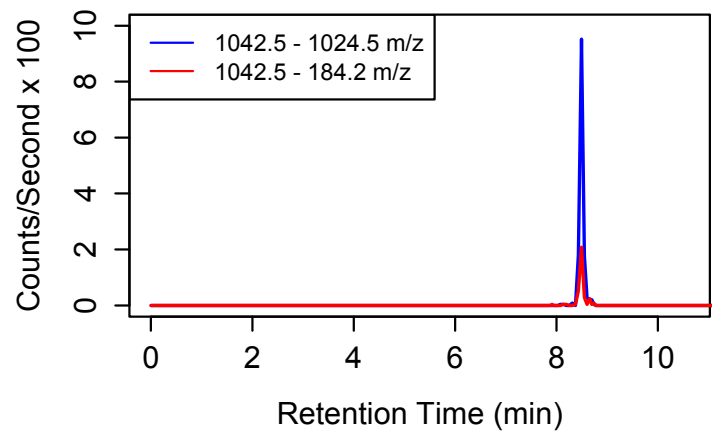

AptB

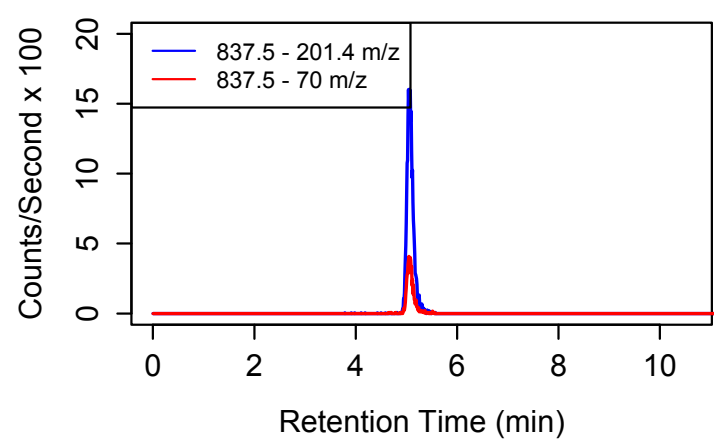

Mgn690

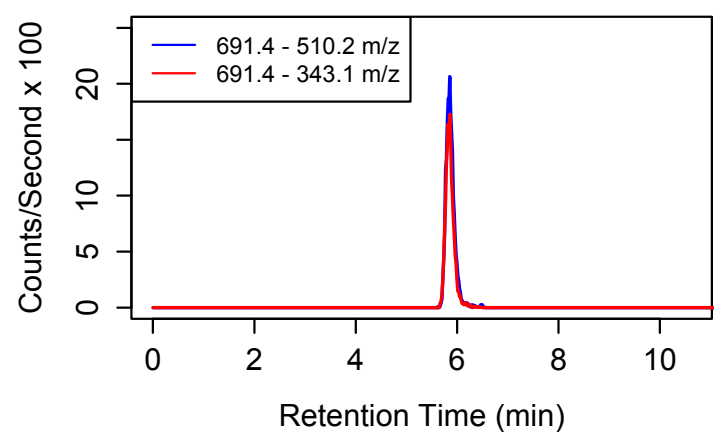

Figure S6. Chromatograms produced from a $10 \mu \mathrm{g} / 1$ standard of cyanopeptolins (Cpt-1007, Cpt1020, and Cpt1041), anabaenopeptins (Apt-A, Apt-B and Apt-F) and microginin-690 (Mgn-690). Also hyphenate the Figure-labels! 


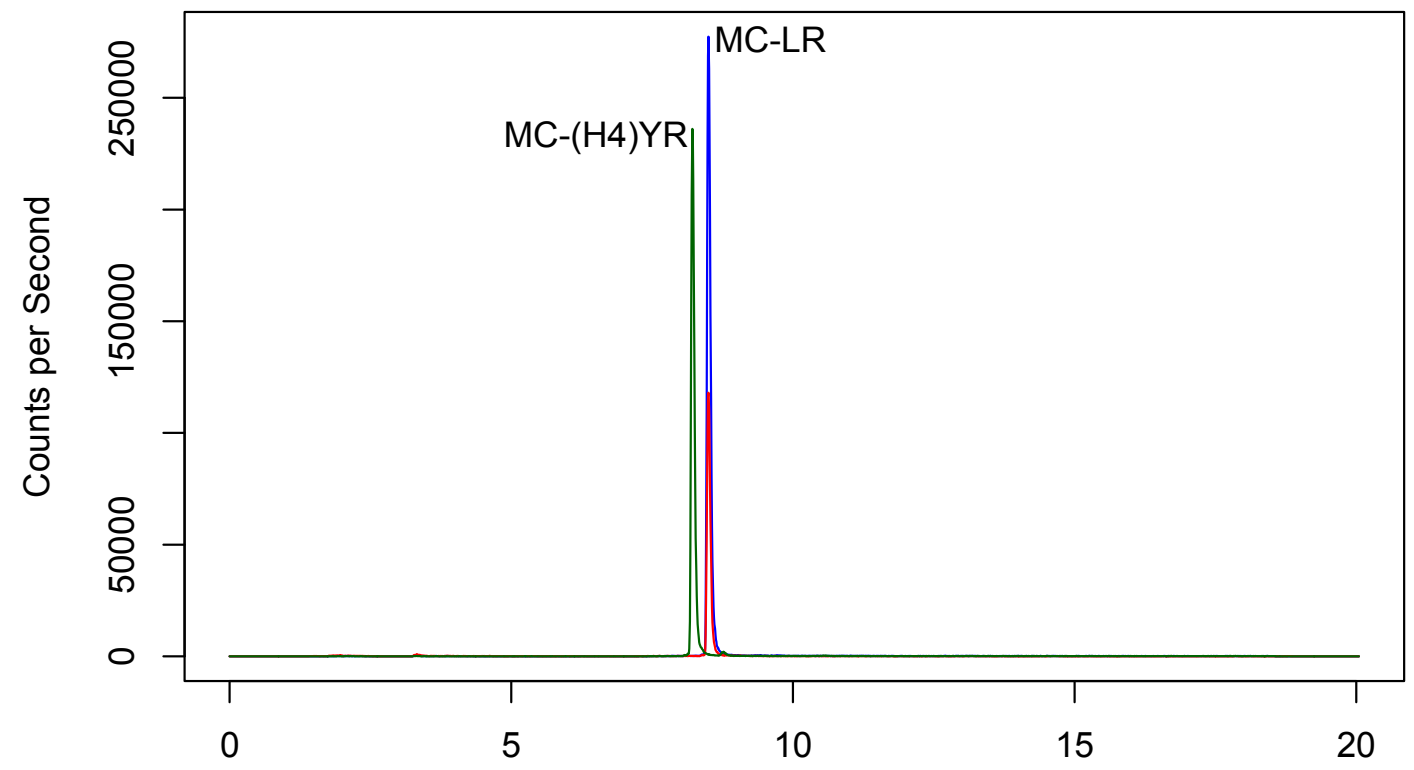

Figure S7. Chromatogram showing multiple reaction monitoring for MC-LR (1) and MC(H4)YR (21) in supplement TM07. 
Table S1. LC-MS/MS Compound-Specific Parameters

\begin{tabular}{|c|c|c|c|c|c|c|c|}
\hline Analyte & Precursor & Product & $\mathrm{RT}^{\mathrm{a}}$ & $\mathrm{DP}^{\mathrm{b}}$ & $\mathrm{EP}^{\mathrm{c}}$ & $C E^{\mathrm{d}}$ & $\mathrm{CXP}^{\mathrm{e}}$ \\
\hline \multirow[t]{2}{*}{ MC-LR } & 995.6 & 135.3 & 8.69 & 126 & 10 & 115 & 26 \\
\hline & & 127.1 & & 126 & 10 & 115 & 26 \\
\hline \multirow[t]{2}{*}{ MC-YR } & 1045.6 & 135.3 & 8.56 & 141 & 10 & 107 & 8 \\
\hline & & 127.1 & & 141 & 10 & 123 & 8 \\
\hline \multirow[t]{2}{*}{ MC-LA } & 910.6 & 776.4 & 9.91 & 106 & 10 & 27 & 8 \\
\hline & & 135.2 & & 106 & 10 & 87 & 8 \\
\hline \multirow[t]{2}{*}{ MC-RR } & 520.0 & 70.1 & 7.80 & 56 & 10 & 129 & 6 \\
\hline & & 135.1 & & 81 & 10 & 43 & 8 \\
\hline \multirow[t]{2}{*}[\mathrm{Dha}^{7}]{$\mathrm{MC}-\mathrm{LR}$} & 981.5 & 135.3 & 8.69 & 126 & 10 & 101 & 22 \\
\hline & & 103.2 & & 126 & 10 & 129 & 6 \\
\hline \multirow[t]{2}{*}{ MC-LF } & 986.5 & 135.3 & 10.57 & 91 & 10 & 91 & 8 \\
\hline & & 375.3 & & 91 & 10 & 49 & 10 \\
\hline \multirow{2}{*}{ MC-LY } & 1002.5 & 135.3 & 9.92 & 96 & 10 & 89 & 8 \\
\hline & & 107.2 & & 96 & 10 & 129 & 18 \\
\hline \multirow[t]{2}{*}{ MC-WR } & 1068.5 & 135.3 & 8.83 & 161 & 10 & 109 & 22 \\
\hline & & 159.4 & & 161 & 10 & 103 & 8 \\
\hline \multirow[t]{2}{*}{ MC-HilR } & 1009.6 & 135.3 & 8.76 & 126 & 10 & 99 & 22 \\
\hline & & 107.2 & & 126 & 10 & 129 & 18 \\
\hline \multirow{2}{*}{ MC-HtyR } & 1059.6 & 135.3 & 8.55 & 136 & 10 & 105 & 8 \\
\hline & & 107.3 & & 136 & 10 & 127 & 18 \\
\hline \multirow[t]{2}{*}{ MC-LW } & 1025.5 & 135.3 & 10.37 & 101 & 10 & 99 & 8 \\
\hline & & 107.2 & & 101 & 10 & 129 & 16 \\
\hline \multirow[t]{2}{*}{ Apt-B } & 837.5 & 201.4 & 4.76 & 106 & 10 & 57 & 14 \\
\hline & & 70.0 & & 106 & 10 & 129 & 10 \\
\hline \multirow[t]{2}{*}{ Apt-F } & 851.8 & 201.0 & 5.46 & 121 & 10 & 53 & 12 \\
\hline & & 175.1 & & 121 & 10 & 53 & 12 \\
\hline \multirow[t]{2}{*}{ Apt-A } & 844.5 & 84.3 & 8.38 & 81 & 10 & 129 & 14 \\
\hline & & 637.4 & & 81 & 10 & 37 & 20 \\
\hline \multirow[t]{2}{*}{ Cpt-1007 } & 1007.5 & 989.6 & 8.30 & 131 & 10 & 51 & 32 \\
\hline & & 776.3 & & 131 & 10 & 59 & 22 \\
\hline \multirow[t]{2}{*}{ Cpt-1041 } & 1042.5 & 1024.5 & 8.56 & 131 & 10 & 51 & 28 \\
\hline & & 184.2 & & 131 & 10 & 109 & 8 \\
\hline \multirow[t]{2}{*}{ Cpt-1020 } & 1021.6 & 989.6 & 8.86 & 131 & 10 & 57 & 32 \\
\hline & & 776.4 & & 131 & 10 & 63 & 22 \\
\hline \multirow[t]{2}{*}{ Mgn-690 } & 691.4 & 510.2 & 5.25 & 96 & 10 & 31 & 16 \\
\hline & & 343.1 & & 96 & 10 & 37 & 10 \\
\hline \multirow[t]{2}{*}{ NOD-R } & 825.5 & 103.2 & 8.30 & 116 & 10 & 83 & 8 \\
\hline & & 135.3 & & 116 & 10 & 129 & 16 \\
\hline
\end{tabular}

${ }^{\mathrm{a}} \mathrm{RT}=$ Retention Time, ${ }^{\mathrm{b}} \mathrm{DP}=$ Declustering Potential, ${ }^{\mathrm{c}} \mathrm{EP}=$ Entrance Potential, ${ }^{\mathrm{d}}$ Collision Energy, ${ }^{\mathrm{e}} \mathrm{CXP}=$ Collision Cell Exit Potential 
Table S2. Non-Compound-Specific LC-MS/MS Parameters

\begin{tabular}{ll}
\hline Parameter & Setting \\
\hline Curtain Gas (psi) & 20 \\
Collision Gas & High \\
Ion Spray Voltage (psi) & 5500 \\
Temperature (C) & 700 \\
Ion Source Gas 1 (psi) & 70 \\
Ion Source Gas 2 (psi) & 70 \\
\hline
\end{tabular}


Table S3. Percent recovery of target analytes after extraction and analysis by LC-MS/MS.

\begin{tabular}{|c|c|c|c|c|c|}
\hline Analyte & TM01 & TM13 & TM18 & Average & SD \\
\hline MC-LR & 97.4 & 102.8 & 100.8 & 100.3 & 2.73 \\
\hline NOD-R & 95.6 & 89.6 & 76.6 & 87.3 & 9.71 \\
\hline MC-YR & 86.6 & 101.8 & 80.8 & 89.7 & 10.84 \\
\hline MC-LA & 95.6 & 84.0 & 89.8 & 89.8 & 5.80 \\
\hline MC-RR & 92.8 & 96.8 & 95.0 & 94.9 & 2.00 \\
\hline Apt-B & 104.6 & 94.8 & 98.0 & 99.1 & 5.00 \\
\hline Apt-F & 99.6 & 95.6 & 99.6 & 98.3 & 2.31 \\
\hline Cpt-1007 & 137.2 & 118.0 & 121.4 & 125.5 & 10.25 \\
\hline Cpt-1020 & 163.4 & 140.0 & 155.4 & 152.9 & 11.89 \\
\hline Mgn-690 & 70.0 & 60.6 & 64.4 & 65.0 & 4.73 \\
\hline$\left[\mathrm{Dha}^{7}\right] \mathrm{MC}-\mathrm{LR}$ & 89.0 & 89.6 & 94.4 & 91.0 & 2.96 \\
\hline MC-LF & 96.4 & 94.6 & 89.2 & 93.4 & 3.75 \\
\hline MC-LY & 98.8 & 89.6 & 93.0 & 93.8 & 4.65 \\
\hline MC-WR & 71.0 & 84.0 & 92.0 & 82.3 & 10.60 \\
\hline MC-HilR & 91.0 & 89.4 & 105.0 & 95.1 & 8.58 \\
\hline MC-HtyR & 90.4 & 81.8 & 93.4 & 88.5 & 6.02 \\
\hline MC-LW & 98.6 & 99.2 & 99.0 & 98.9 & 0.31 \\
\hline
\end{tabular}



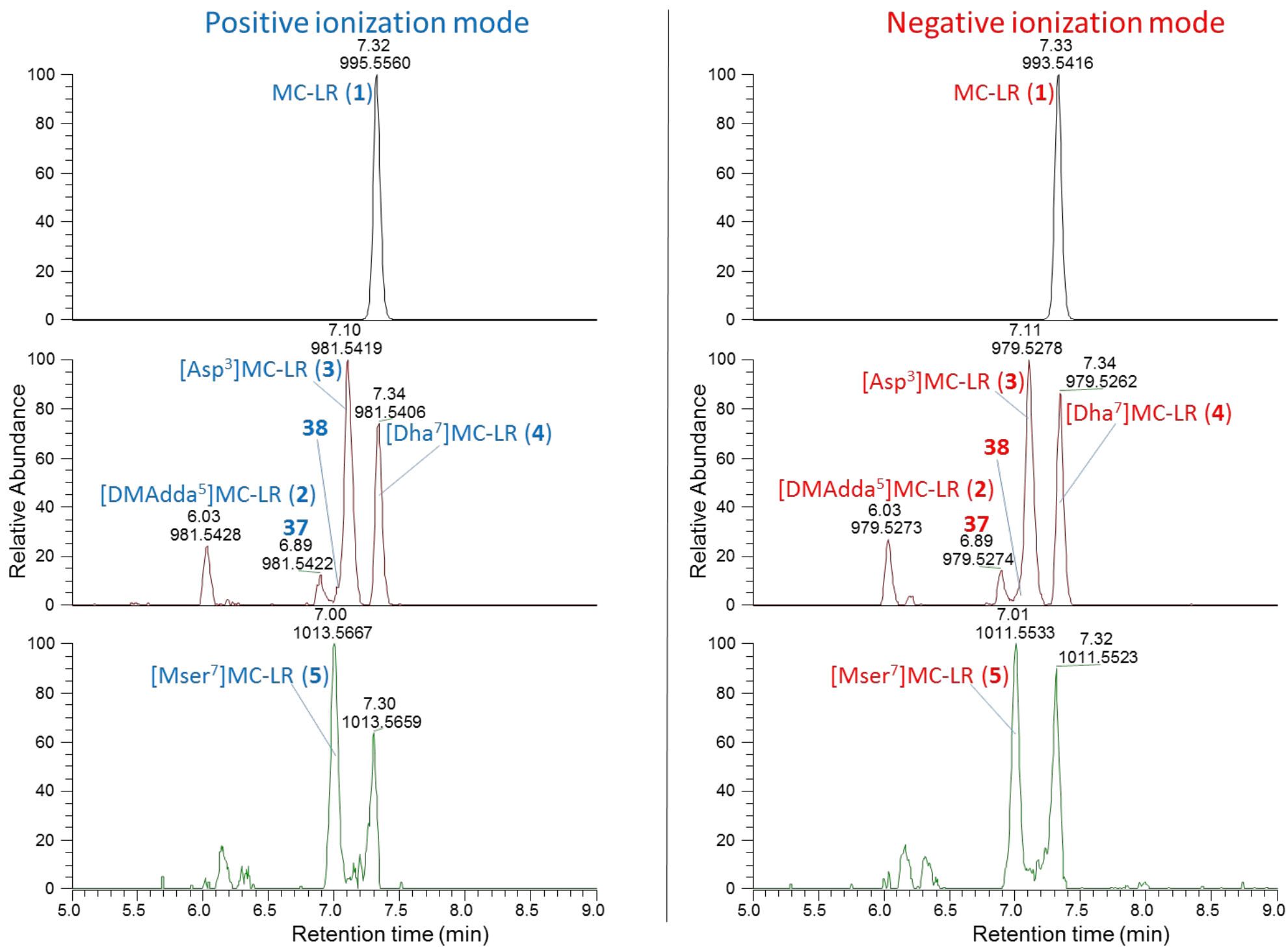

Figure S8. Full-scan LC-HRMS chromatograms extracted at the exact $[\mathrm{M}+\mathrm{H}]^{+}$or $[\mathrm{M}-\mathrm{H}]^{-} m / z$ for: top, MC-LR (1); middle, desmethylMC-LR, and; bottom, [Mser $\left.{ }^{7}\right] \mathrm{MC}-\mathrm{LR}$ (5), in positive (left) and negative (right) ionization modes. 

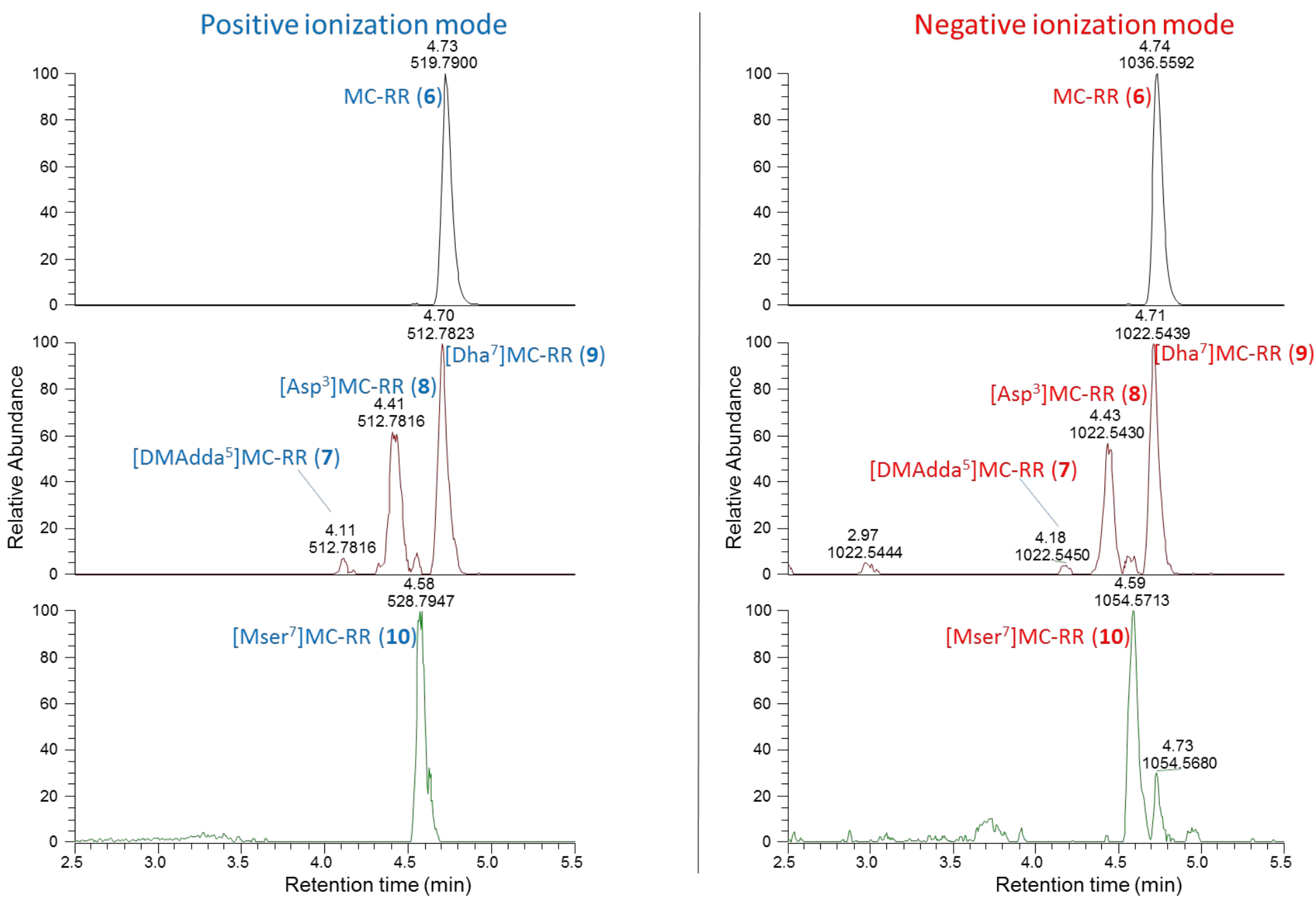

Figure S9. Full-scan LC-HRMS chromatograms extracted at the exact $[\mathrm{M}+\mathrm{H}]^{+}$or $[\mathrm{M}-\mathrm{H}]^{-} \mathrm{m} / z$ for: top, MC-RR (6); middle, desmethylMC-RR, and; bottom, [Mser $\left.{ }^{7}\right] \mathrm{MC}-\mathrm{RR}(\mathbf{1 0})$, in positive (left) and negative (right) ionization modes. 

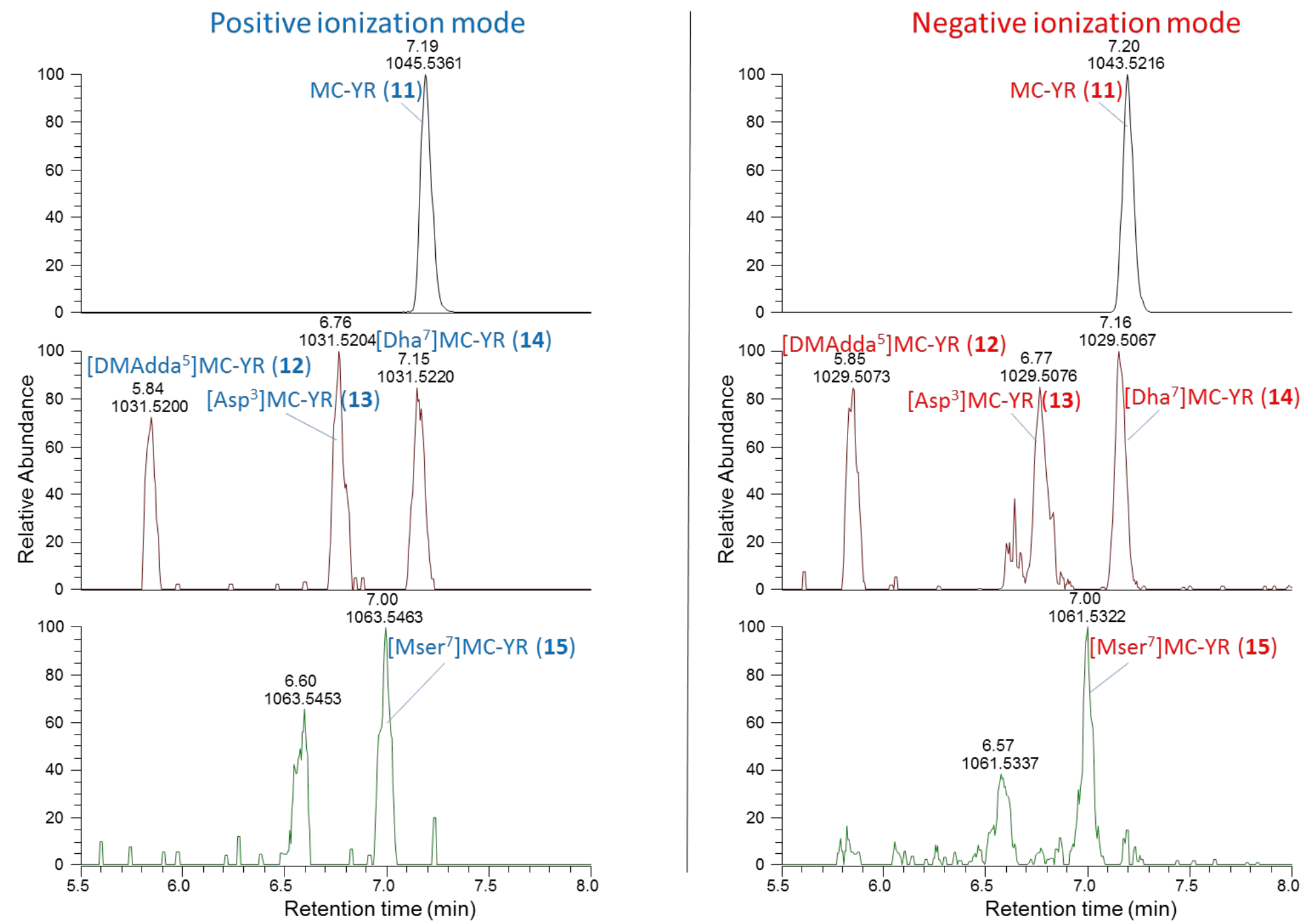

Figure S10. Full-scan LC-HRMS chromatograms extracted at the exact $[\mathrm{M}+\mathrm{H}]^{+}$or $[\mathrm{M}-\mathrm{H}]^{-} \mathrm{m} / z$ for: top, MC-YR (11); middle, desmethylMC-YR, and; bottom, [Mser $\left.{ }^{7}\right] \mathrm{MC}-\mathrm{YR}$ (15), in positive (left) and negative (right) ionization modes. 

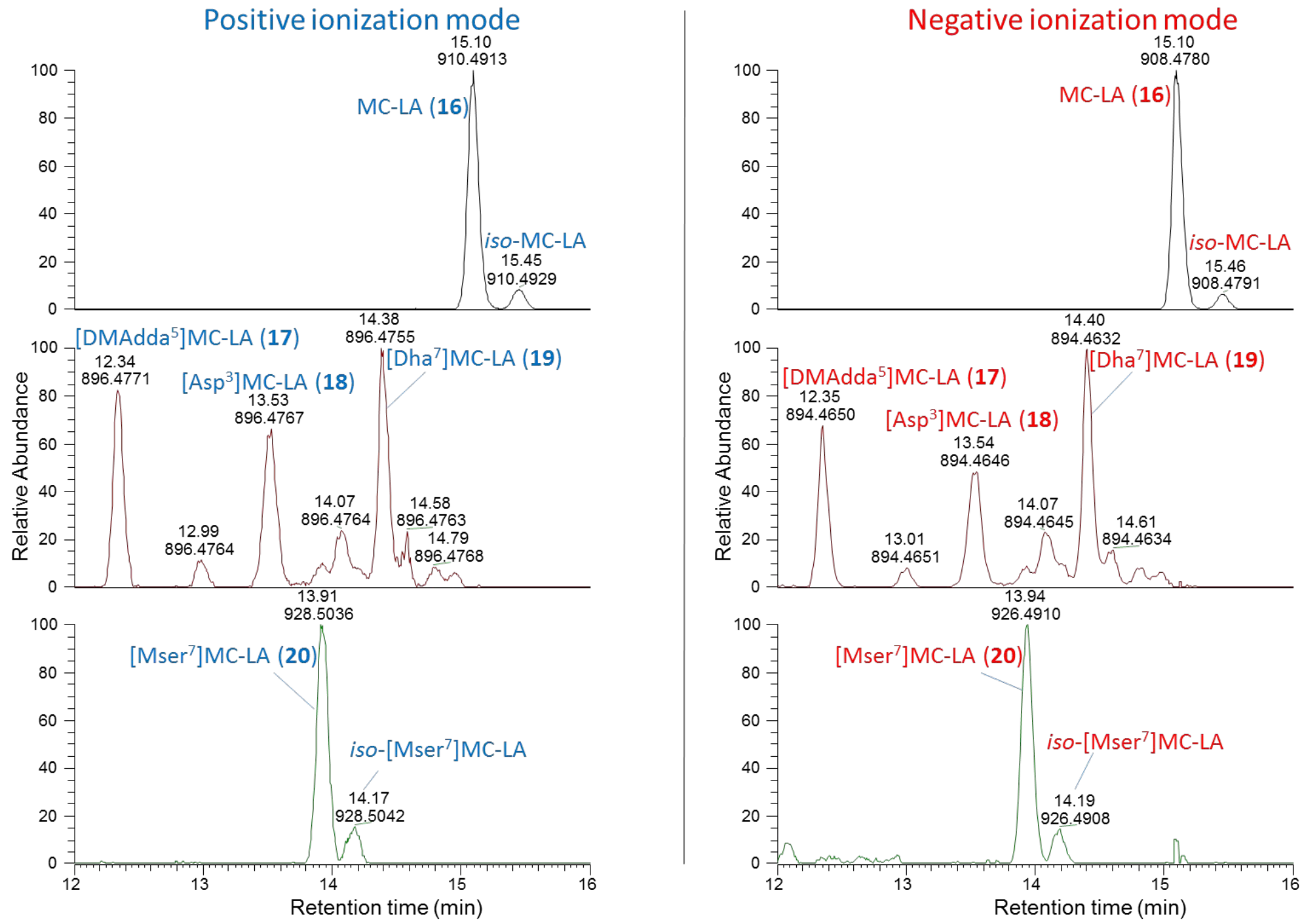

Figure S11. Full-scan LC-HRMS chromatograms extracted at the exact $[\mathrm{M}+\mathrm{H}]^{+}$or $[\mathrm{M}-\mathrm{H}]^{-} \mathrm{m} / z$ for: top, MC-LA (16); middle, desmethylMC-LA, and; bottom, [Mser $\left.{ }^{7}\right] \mathrm{MC}-\mathrm{LA}(\mathbf{2 0})$, in positive (left) and negative (right) ionization modes. 

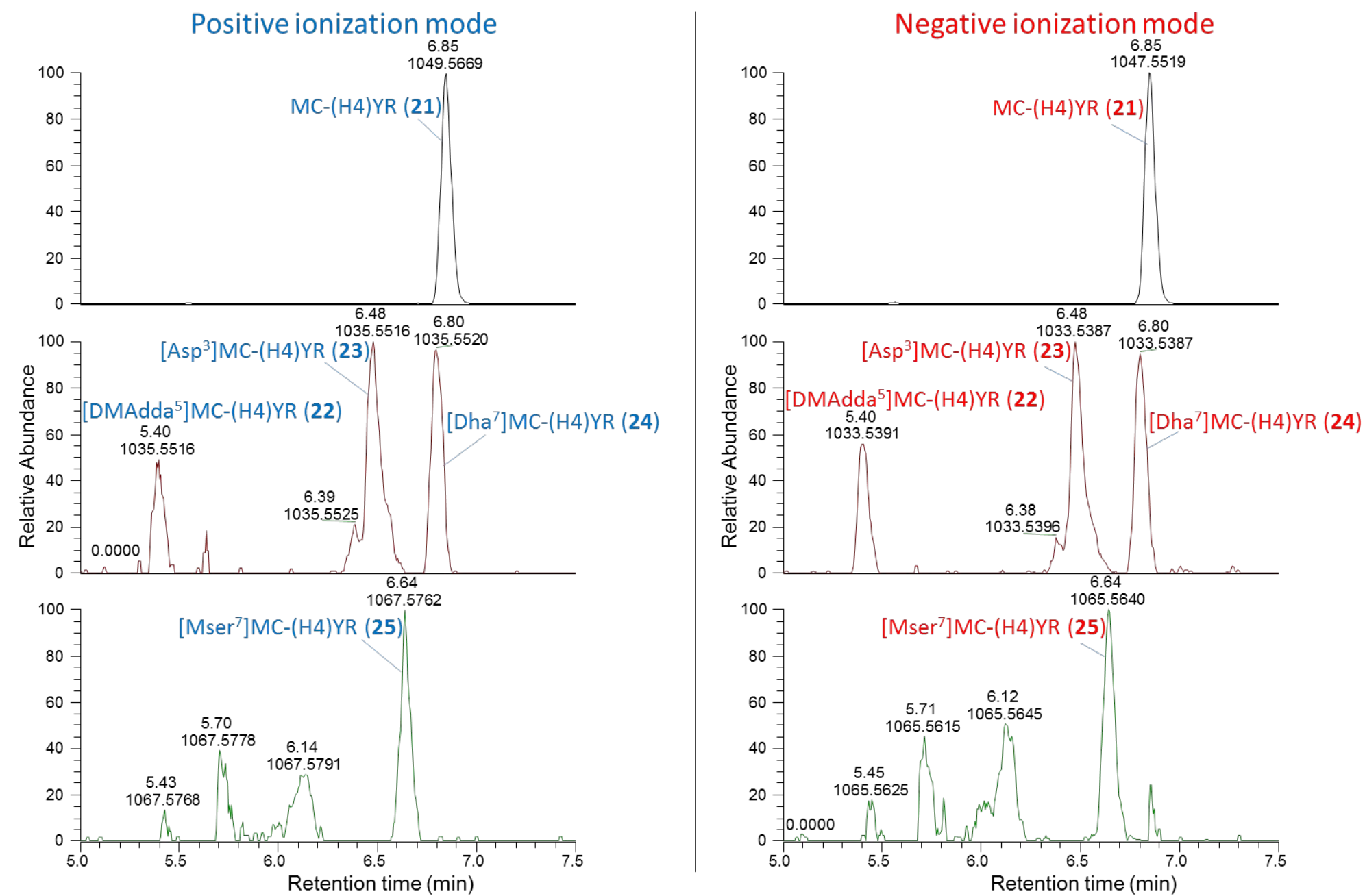

Figure S12. Full-scan LC-HRMS chromatograms extracted at the exact $[\mathrm{M}+\mathrm{H}]^{+}$or $[\mathrm{M}-\mathrm{H}]^{-} \mathrm{m} / z$ for: top, MC-(H4)YR (21); middle, desmethylMC-(H4)YR, and; bottom, [Mser ${ }^{7}$ MC-(H4)YR (25), in positive (left) and negative (right) ionization modes. 

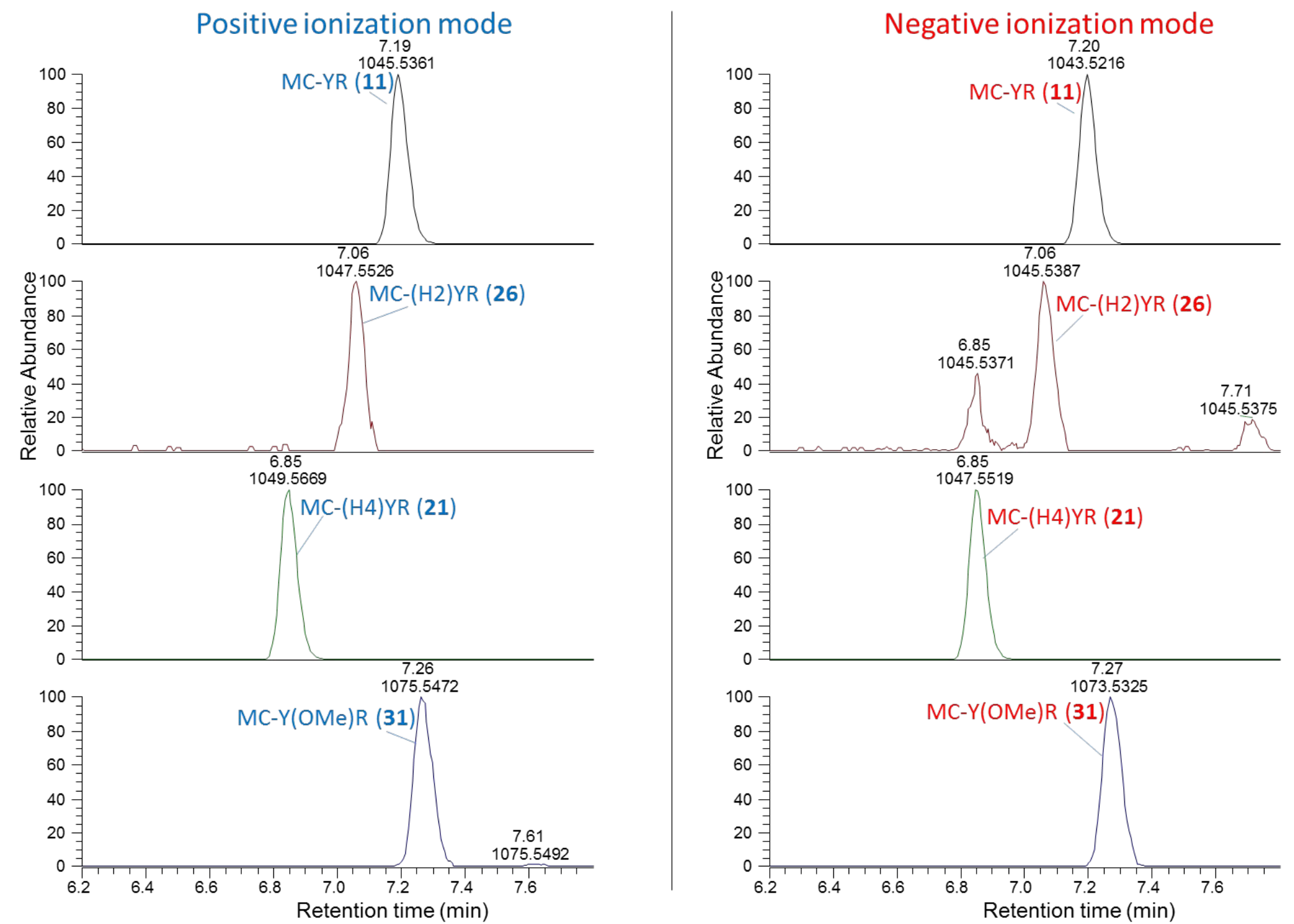

Figure S13. Full-scan LC-HRMS chromatograms extracted at the exact $[\mathrm{M}+\mathrm{H}]^{+}$or $[\mathrm{M}-\mathrm{H}]^{-} m / z$ for: top, MC-YR (11); $2^{\text {nd }}, \mathrm{MC}-$ (H2)YR (26); $3^{\text {rd }}$, MC-(H4)YR (21), and; bottom, MC-Y(OMe)YR (31), in positive (left) and negative (right) ionization modes. 

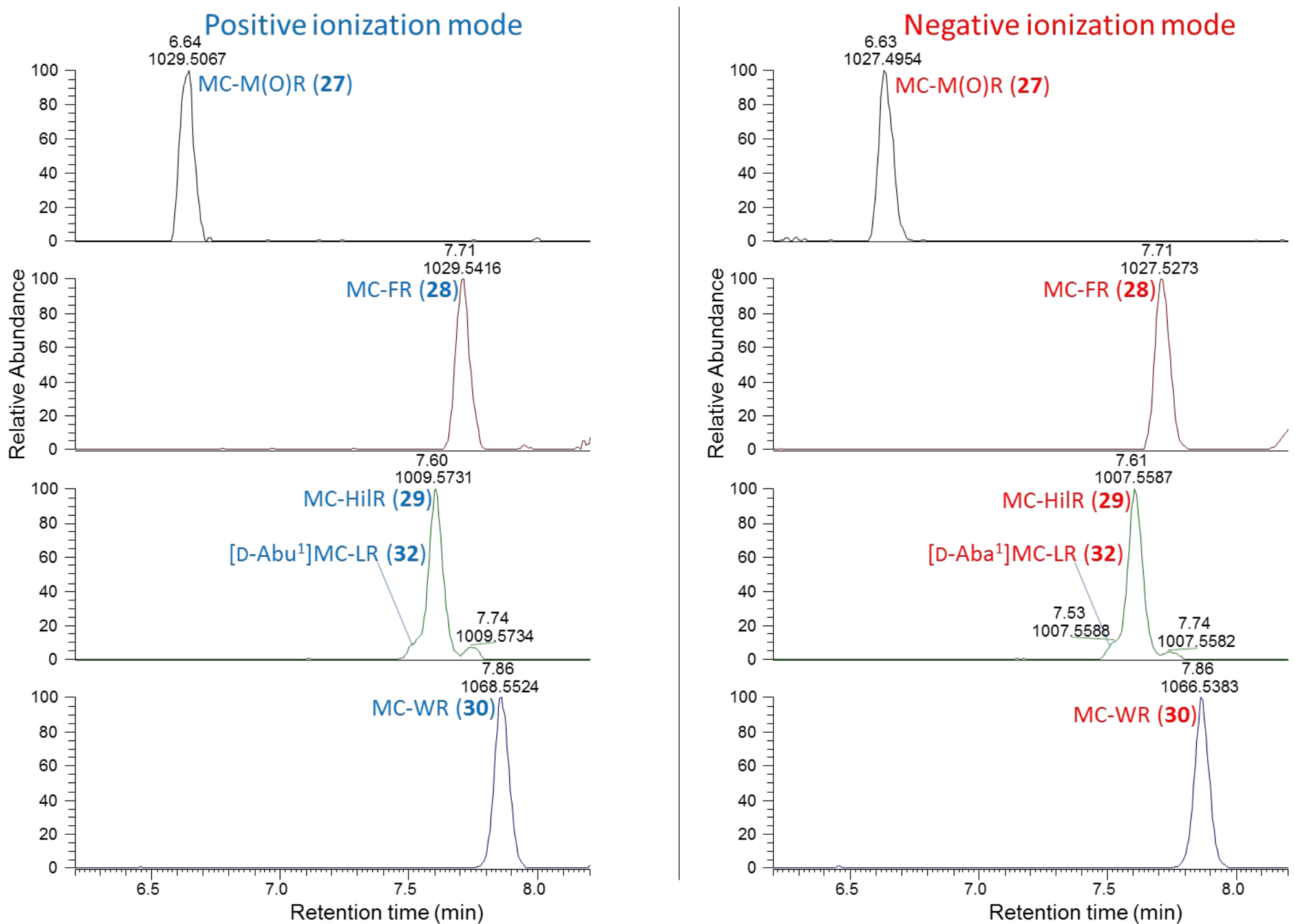

Figure S14. Full-scan LC-HRMS chromatograms extracted at the exact $[\mathrm{M}+\mathrm{H}]^{+}$or $[\mathrm{M}-\mathrm{H}]^{-} m / z$ for: top, $\mathrm{MC}-\mathrm{M}(\mathrm{O}) \mathrm{R}(\mathbf{2 7}) ; 2^{\text {nd }}, \mathrm{MC}-$ FR (26); $3^{\text {rd }}$, MC-HilR (29) and [D-Abu $\left.{ }^{1}\right]$ MC-LR (32), and; bottom, MC-WR (30), in positive (left) and negative (right) ionization modes. 

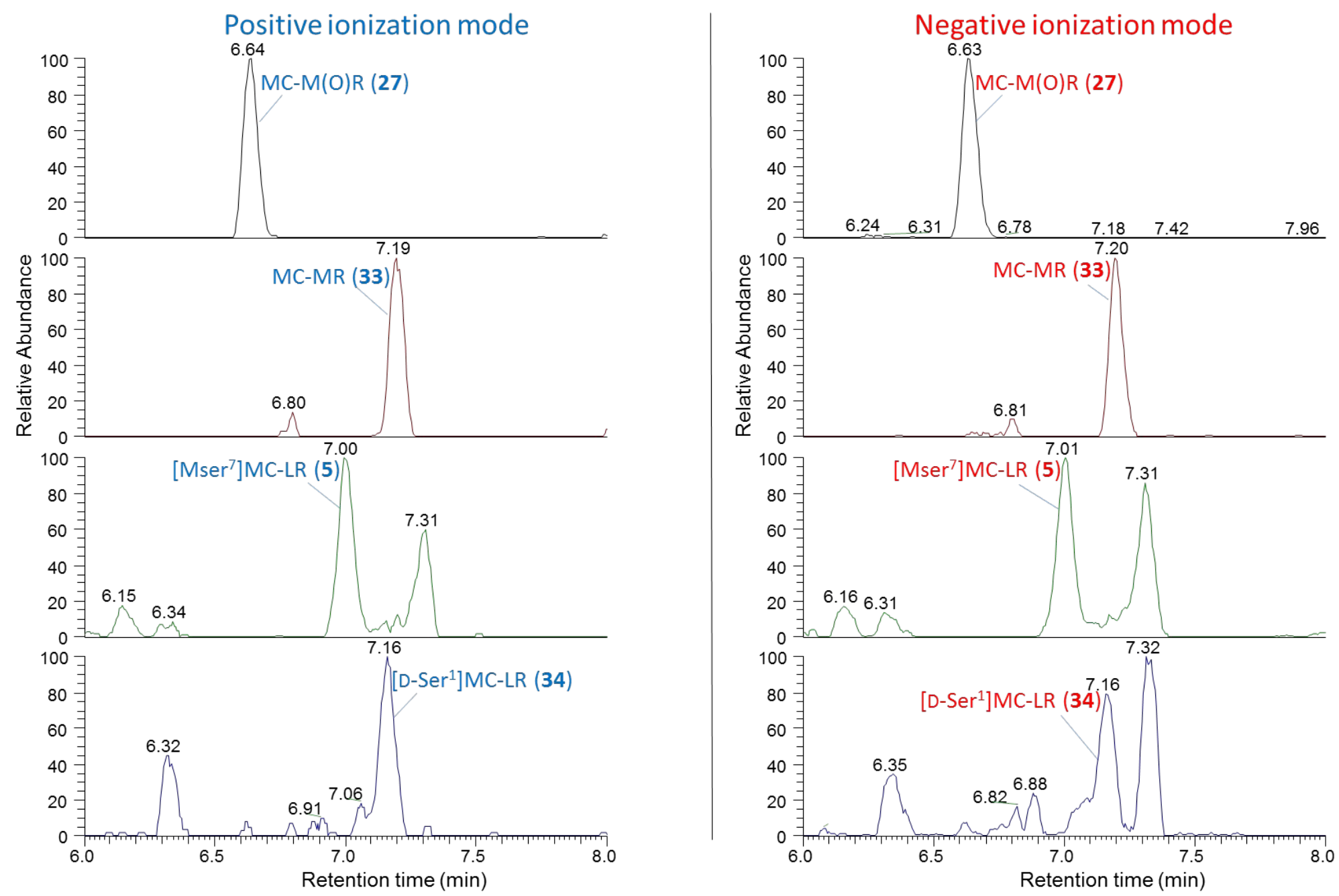

Figure S15. Full-scan LC-HRMS chromatograms extracted at the exact $[\mathrm{M}+\mathrm{H}]^{+}$or $[\mathrm{M}-\mathrm{H}]^{-} m / z$ for: top, $\mathrm{MC}-\mathrm{M}(\mathrm{O}) \mathrm{R}(\mathbf{2 7})$; $2^{\text {nd }}, \mathrm{MC}-$ MR (33); $3^{\text {rd }},\left[\mathrm{Mser}^{7}\right] \mathrm{MC}-\mathrm{LR}(\mathbf{5})$ and; bottom, D-Ser ${ }^{1}$ MC-LR (34), in positive (left) and negative (right) ionization modes. 

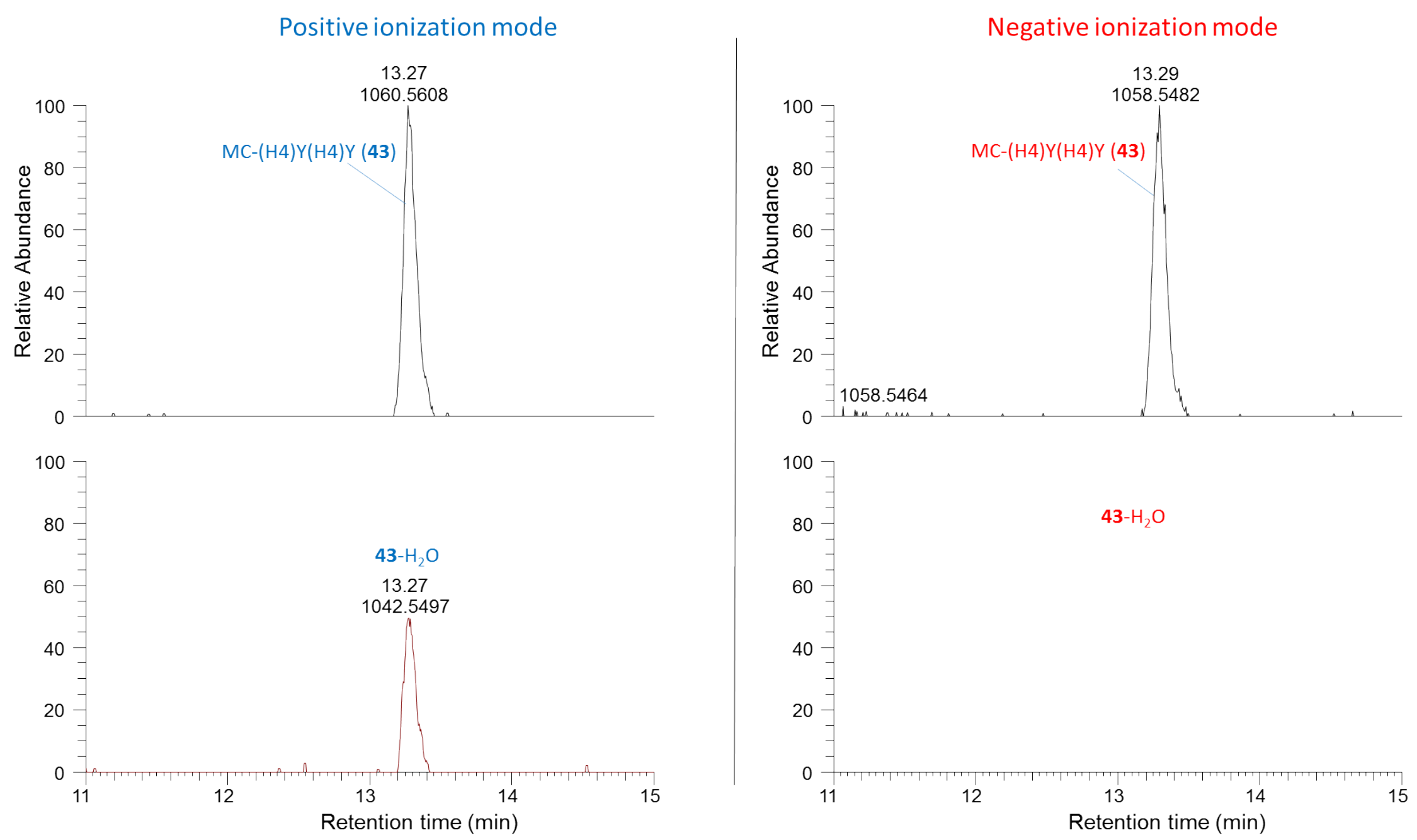

Figure S16. Full-scan LC-HRMS chromatograms extracted at the exact $[\mathrm{M}+\mathrm{H}]^{+}$or $[\mathrm{M}-\mathrm{H}]^{-} \mathrm{m} / z$ for: top, MC-(H4)Y(H4Y) (43), and; bottom, water loss from MC-(H4)Y(H4Y) (43), in positive (left) and negative (right) ionization modes. Each of the bottom chromatograms is plotted on the same vertical scale as the chromatogram above it, and illustrates abundant water loss from $[\mathrm{M}+\mathrm{H}]^{+}$of 43 in positive, but not negative, ionization modes. 

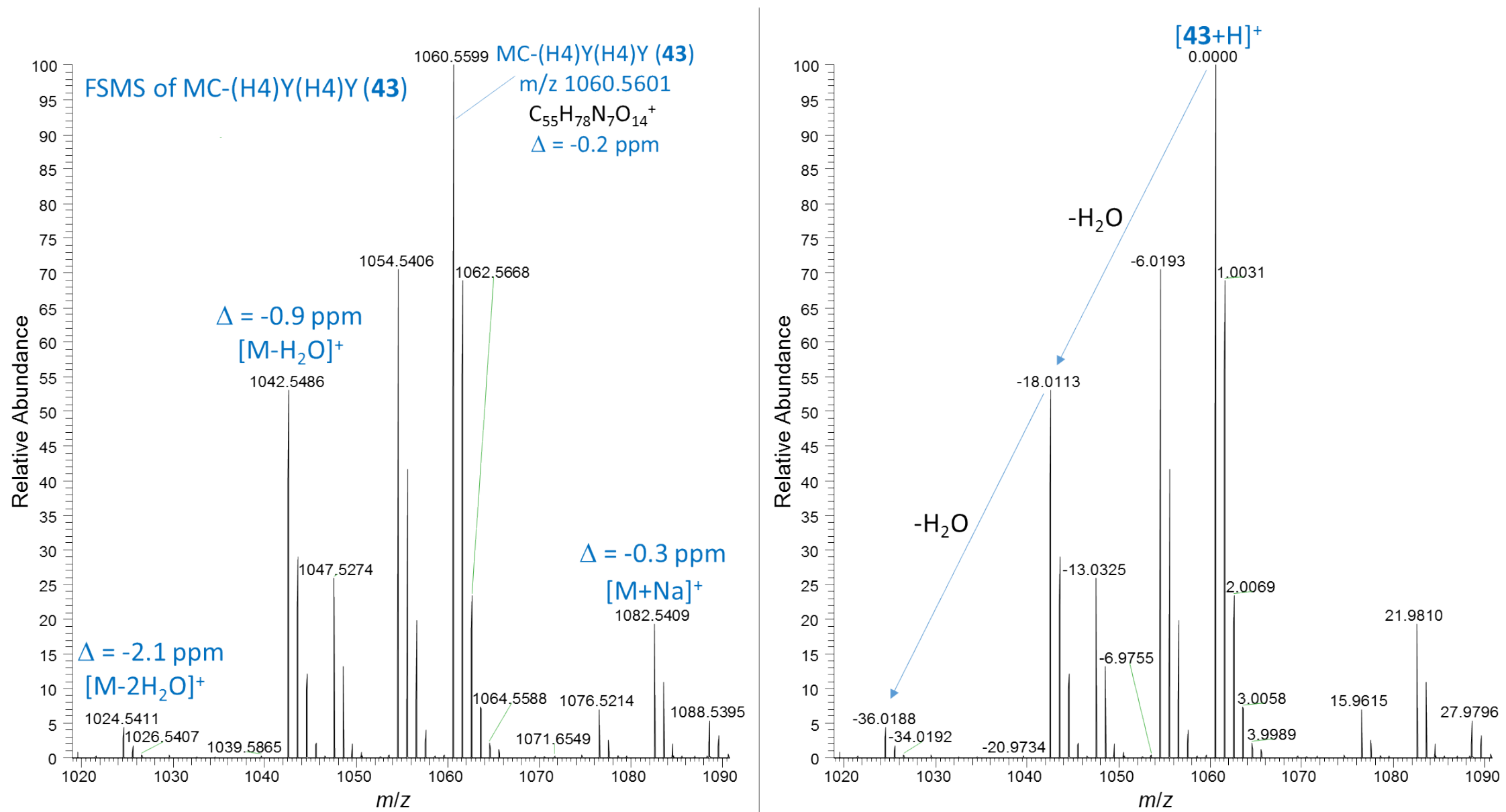

Figure S17. Full scan HRMS spectrum of MC-(H4)Y(H4)Y (43) showing prominent water losses from $[\mathrm{M}+\mathrm{H}]^{+}$. The spectrum on the right is the same as the one on the left, but with $m / z$ labeled relative to $[\mathrm{M}+\mathrm{H}]^{+}$of $\mathbf{4 3}$. 


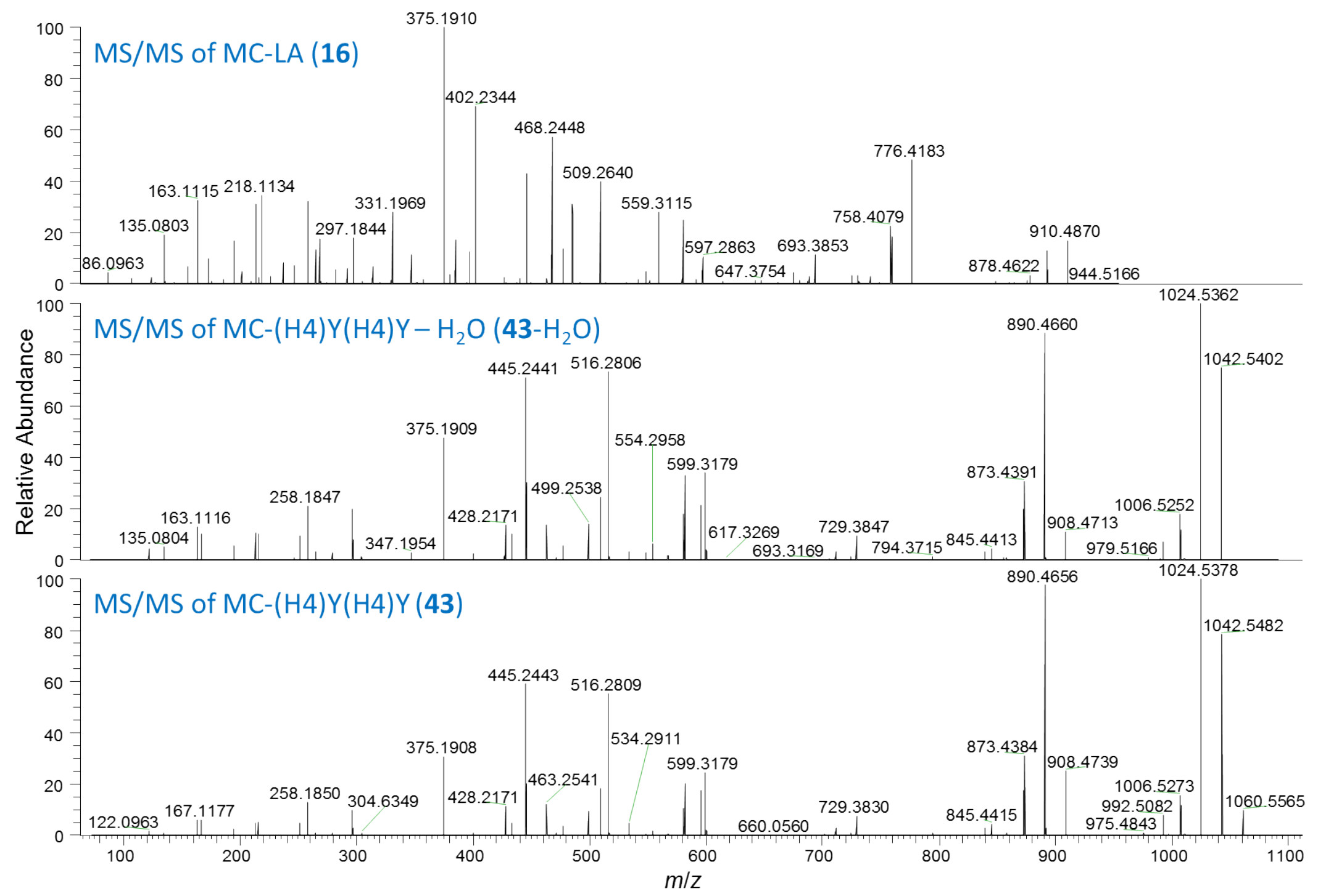

Figure S18. HRMS/MS spectra of: top, $[\mathrm{M}+\mathrm{H}]^{+}$of MC-LA at $m / z 910.5(\mathbf{1 6})$; middle, the water loss peak from $[\mathrm{M}+\mathrm{H}]^{+}$of $\mathbf{4 3}$ at $m / z$ 1042.5; bottom, $[\mathrm{M}+\mathrm{H}]^{+}$of $\mathbf{4 3}$ at $\mathrm{m} / z$ 1060.5. The two spectra from $\mathbf{4 3}$ are virtually indistinguishable apart from a weak $[\mathrm{M}+\mathrm{H}]^{+}$in the bottom spectrum. 


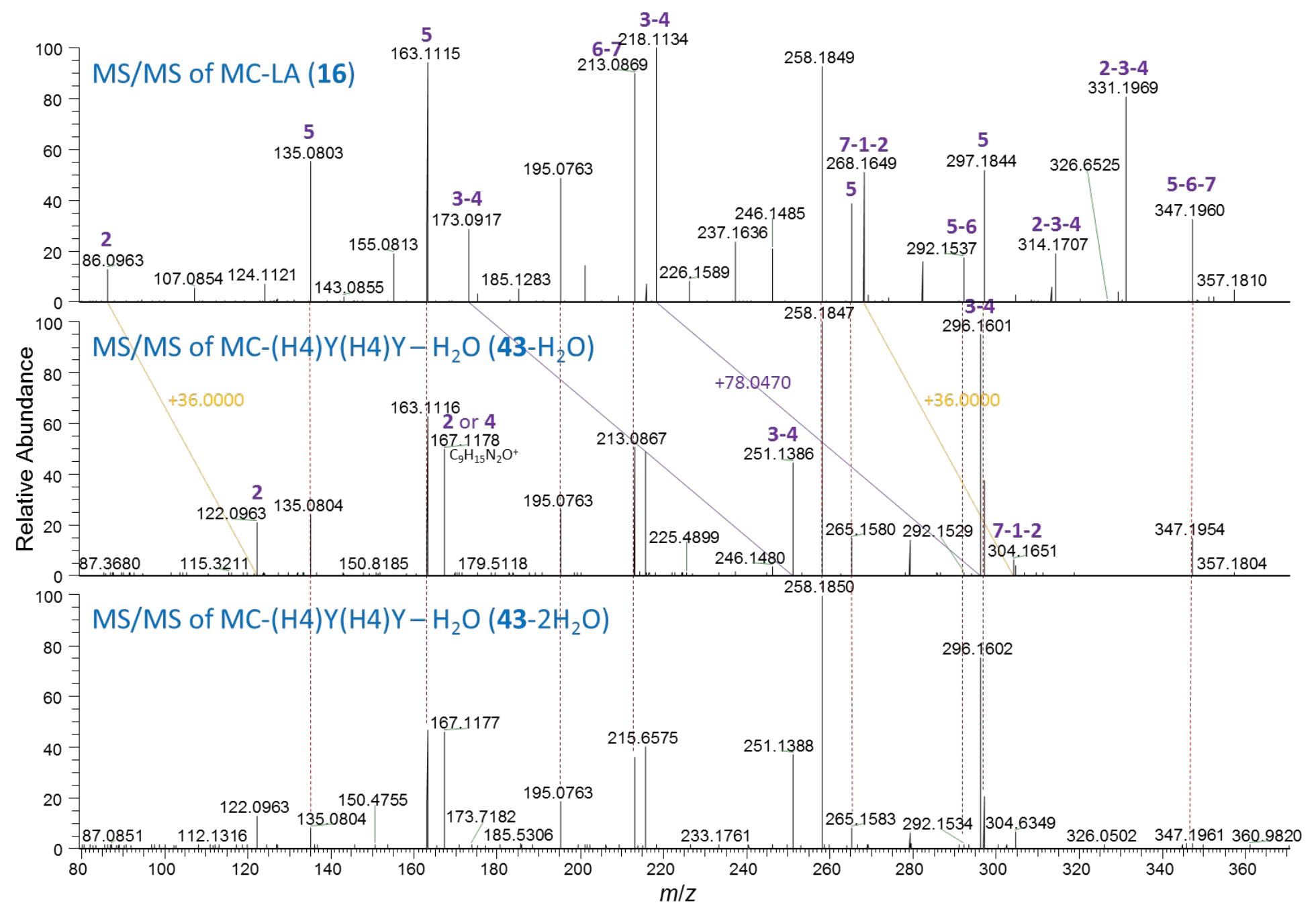

Figure S19. Expansion of the HRMS/MS spectra of: top, $[\mathrm{M}+\mathrm{H}]^{+}$of MC-LA at $\mathrm{m} / \mathrm{z} 910.5$ (16); middle, the water loss peak from $[\mathrm{M}+\mathrm{H}]^{+}$of 43 at $\mathrm{m} / \mathrm{z}$ 1042.5; bottom, $[\mathrm{M}+\mathrm{H}]^{+}$of $\mathbf{4 3}$ at $\mathrm{m} / z$ 1060.5. Purple lines connect ions that change by $\mathrm{m} / z 78.0470\left(\mathrm{Ala}{ }^{4} \rightarrow\right.$ $\left.(\mathrm{H} 4) \mathrm{Tyr}^{4}\right)$, orange lines connect ions that change by $m / z 36.0000\left(\mathrm{Leu}^{2} \rightarrow\left(\mathrm{H}_{4}\right) \mathrm{Tyr}^{2}\right)$, green lines connect ions that change by $m / z$ 114.0470 ( $\mathrm{Leu}^{2}$ and $\mathrm{Ala}^{4} \rightarrow(\mathrm{H} 4) \mathrm{Tyr}^{2}$ and $\left.(\mathrm{H} 4) \mathrm{Tyr}^{4}\right)$, and red dashed lines connect ions that do not change between 16 and 43 . Bold purple numbers indicate the amino acids believed to contribute to selected ions in $\mathbf{1 6}$ and $\mathbf{4 3}$. 


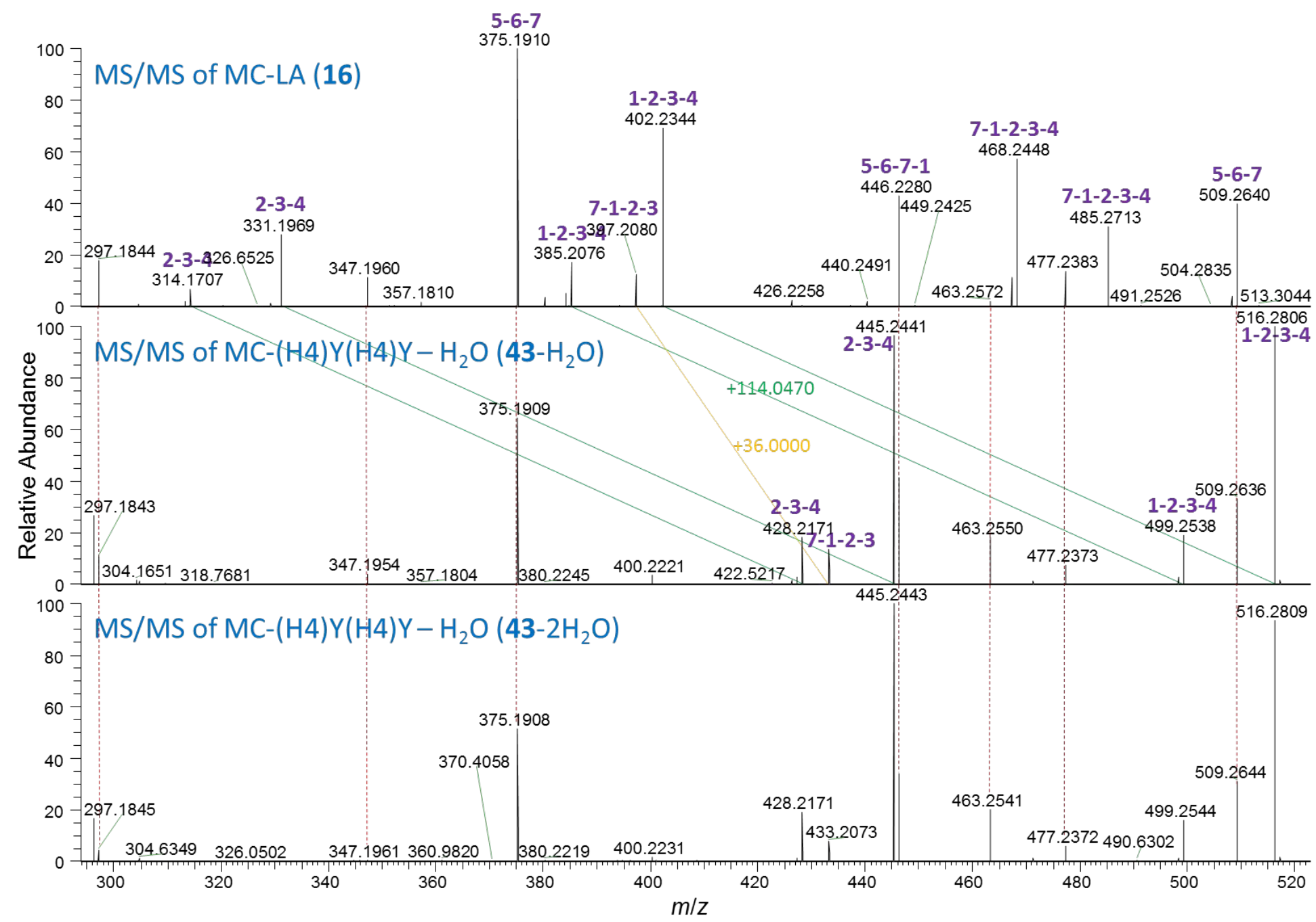

Figure S20. Expansion of the HRMS/MS spectra of: top, $[\mathrm{M}+\mathrm{H}]^{+}$of MC-LA at $\mathrm{m} / \mathrm{z} 910.5$ (16); middle, the water loss peak from $[\mathrm{M}+\mathrm{H}]^{+}$of 43 at $\mathrm{m} / \mathrm{z}$ 1042.5; bottom, $[\mathrm{M}+\mathrm{H}]^{+}$of 43 at $\mathrm{m} / \mathrm{z}$ 1060.5. Purple lines connect ions that change by $\mathrm{m} / \mathrm{z} 78.0470\left(\mathrm{Ala}{ }^{4} \rightarrow\right.$ $\left.(\mathrm{H} 4) \mathrm{Tyr}^{4}\right)$, orange lines connect ions that change by $m / z 36.0000\left(\mathrm{Leu}^{2} \rightarrow\left(\mathrm{H}_{4}\right) \mathrm{Tyr}^{2}\right)$, green lines connect ions that change by $m / z$ $114.0470\left(\mathrm{Leu}^{2}\right.$ and $\mathrm{Ala}^{4} \rightarrow(\mathrm{H} 4) \mathrm{Tyr}^{2}$ and $\left.(\mathrm{H} 4) \mathrm{Tyr}^{4}\right)$, and red dashed lines connect ions that do not change between 16 and 43 . Bold purple numbers indicate the amino acids believed to contribute to selected ions in $\mathbf{1 6}$ and $\mathbf{4 3}$. 


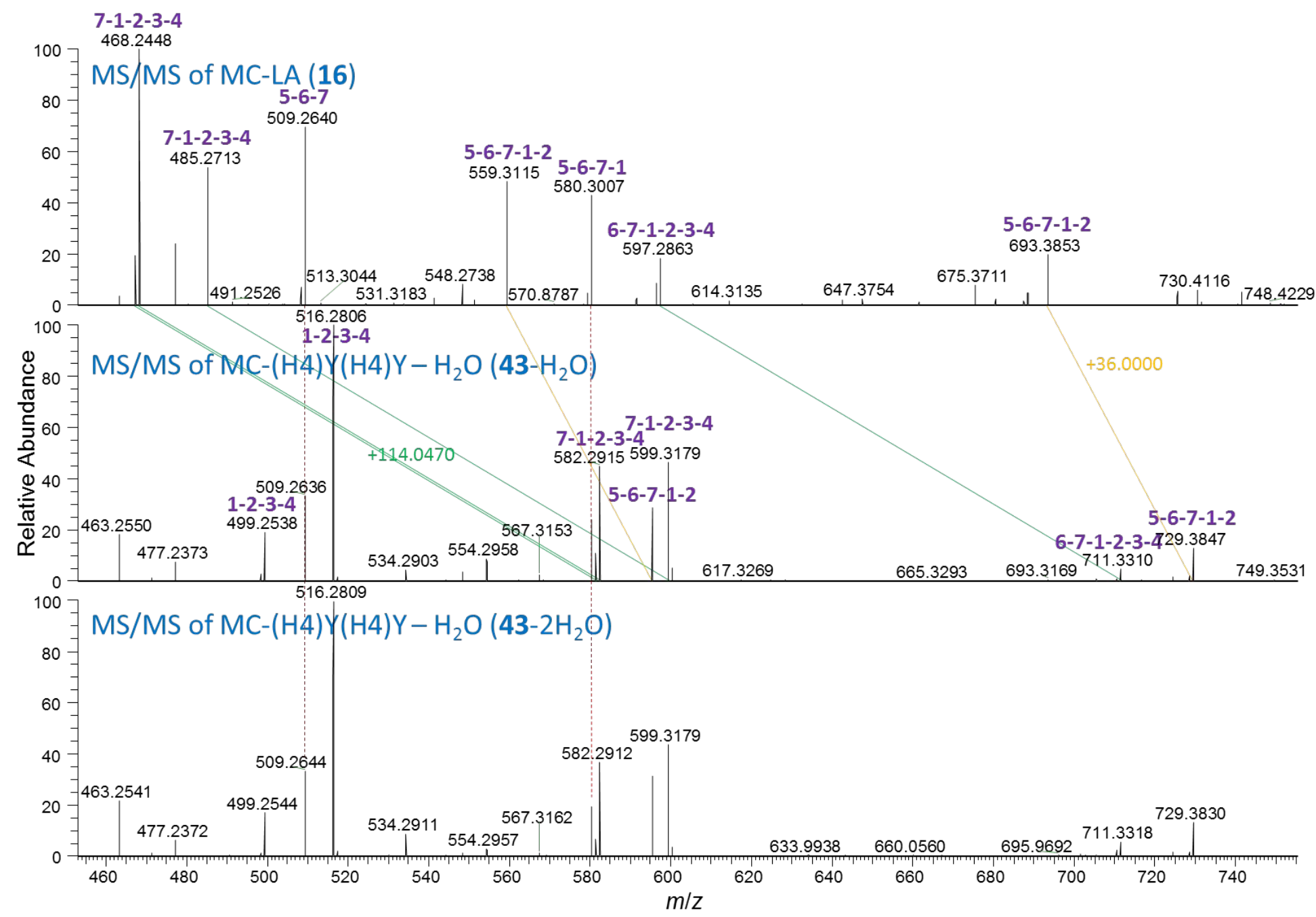

Figure S21. Expansion of the HRMS/MS spectra of: top, $[\mathrm{M}+\mathrm{H}]^{+}$of MC-LA at $\mathrm{m} / \mathrm{z} 910.5$ (16); middle, the water loss peak from $[\mathrm{M}+\mathrm{H}]^{+}$of 43 at $\mathrm{m} / \mathrm{z}$ 1042.5; bottom, $[\mathrm{M}+\mathrm{H}]^{+}$of $\mathbf{4 3}$ at $\mathrm{m} / z$ 1060.5. Purple lines connect ions that change by $\mathrm{m} / z 78.0470\left(\mathrm{Ala}{ }^{4} \rightarrow\right.$ $\left.(\mathrm{H} 4) \mathrm{Tyr}^{4}\right)$, orange lines connect ions that change by $m / z 36.0000\left(\mathrm{Leu}^{2} \rightarrow\left(\mathrm{H}_{4}\right) \mathrm{Tyr}^{2}\right)$, green lines connect ions that change by $m / z$ 114.0470 ( $\mathrm{Leu}^{2}$ and $\mathrm{Ala}^{4} \rightarrow(\mathrm{H} 4) \mathrm{Tyr}^{2}$ and $\left.(\mathrm{H} 4) \mathrm{Tyr}^{4}\right)$, and red dashed lines connect ions that do not change between 16 and 43 . Bold purple numbers indicate the amino acids believed to contribute to selected ions in $\mathbf{1 6}$ and $\mathbf{4 3}$. 


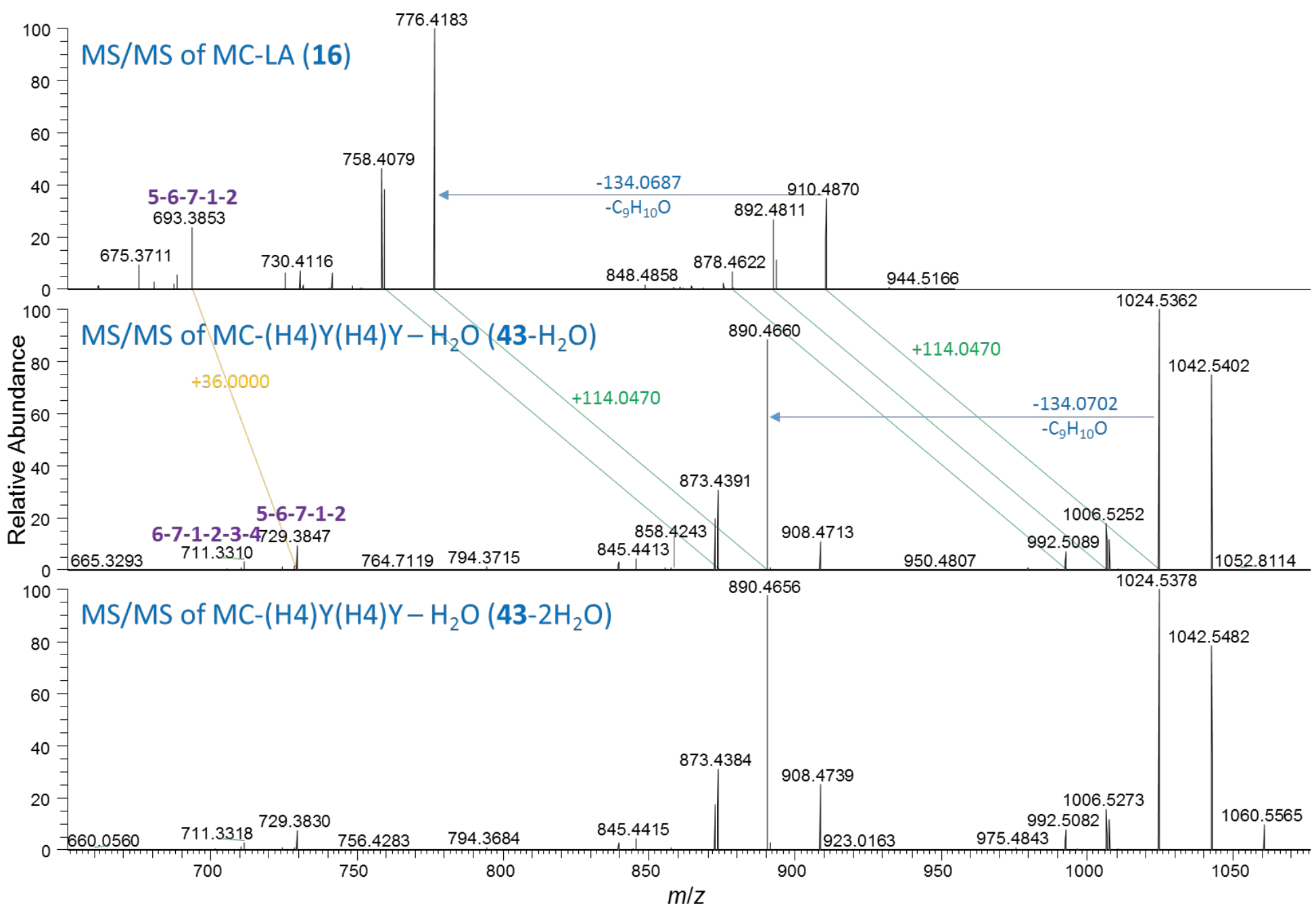

Figure S22. Expansion of the HRMS/MS spectra of: top, $[\mathrm{M}+\mathrm{H}]^{+}$of MC-LA at $\mathrm{m} / \mathrm{z} 910.5$ (16); middle, the water loss peak from $[\mathrm{M}+\mathrm{H}]^{+}$of 43 at $\mathrm{m} / z$ 1042.5; bottom, $[\mathrm{M}+\mathrm{H}]^{+}$of 43 at $\mathrm{m} / \mathrm{z}$ 1060.5. Purple lines connect ions that change by $\mathrm{m} / \mathrm{z} 78.0470\left(\mathrm{Ala}{ }^{4} \rightarrow\right.$ $\left.(\mathrm{H} 4) \mathrm{Tyr}^{4}\right)$, orange lines connect ions that change by $m / z 36.0000\left(\mathrm{Leu}^{2} \rightarrow\left(\mathrm{H}_{4}\right) \mathrm{Tyr}^{2}\right)$, green lines connect ions that change by $m / z$ 114.0470 ( $\mathrm{Leu}^{2}$ and $\mathrm{Ala}^{4} \rightarrow(\mathrm{H} 4) \mathrm{Tyr}^{2}$ and $\left.(\mathrm{H} 4) \mathrm{Tyr}^{4}\right)$, and red dashed lines connect ions that do not change between 16 and 43 . Bold purple numbers indicate the amino acids believed to contribute to selected ions in $\mathbf{1 6}$ and $\mathbf{4 3}$. 


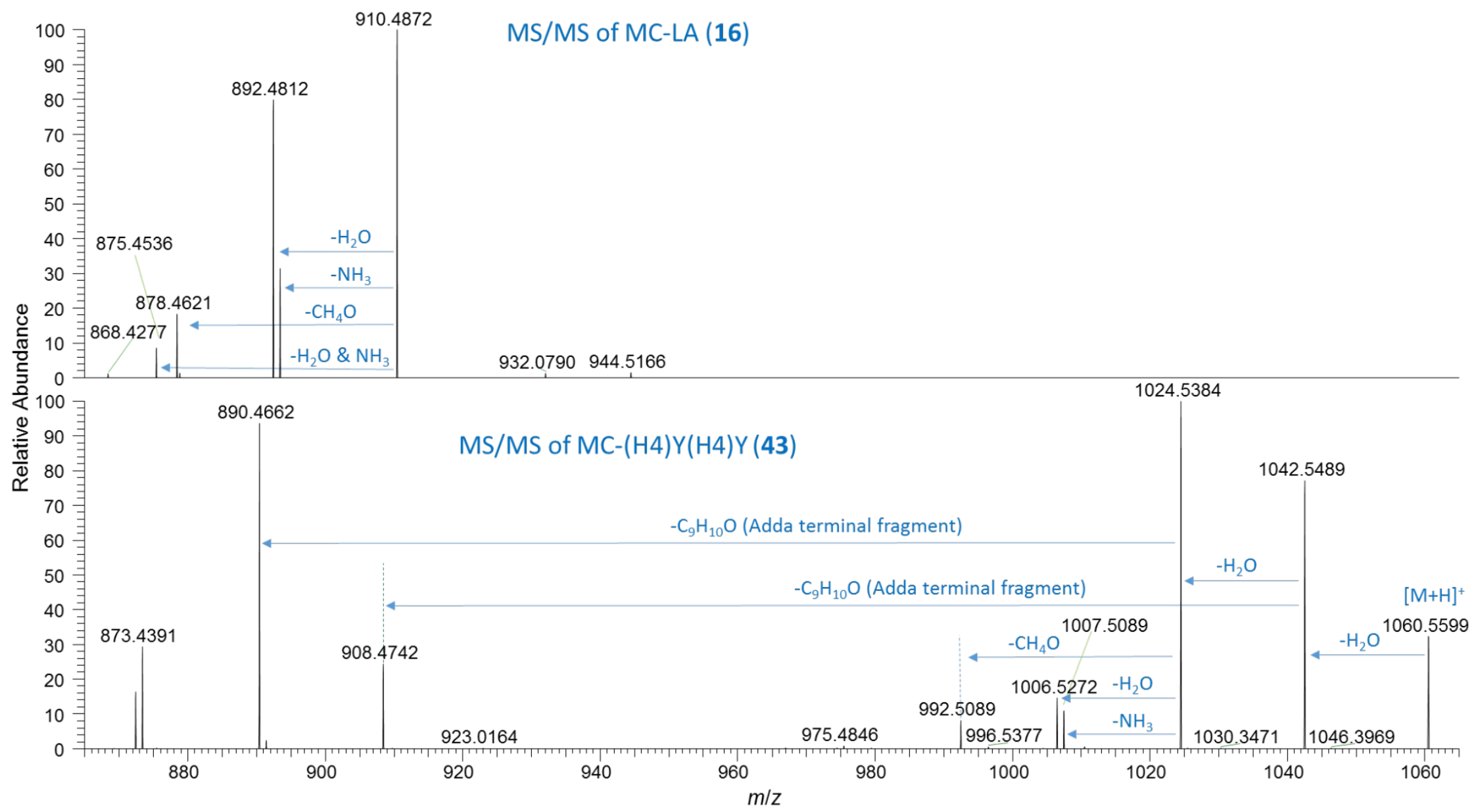

Figure S23. Expansion of the high-mass region of the HRMS/MS spectra of: top, $[\mathrm{M}+\mathrm{H}]^{+}$of MC-LA at $m / z$ 910.5 (16), and; bottom, the water loss peak from $[\mathrm{M}+\mathrm{H}]^{+}$of $\mathbf{4 3}$ at $\mathrm{m} / \mathrm{z}$ 1042.5, showing major neutral losses. 


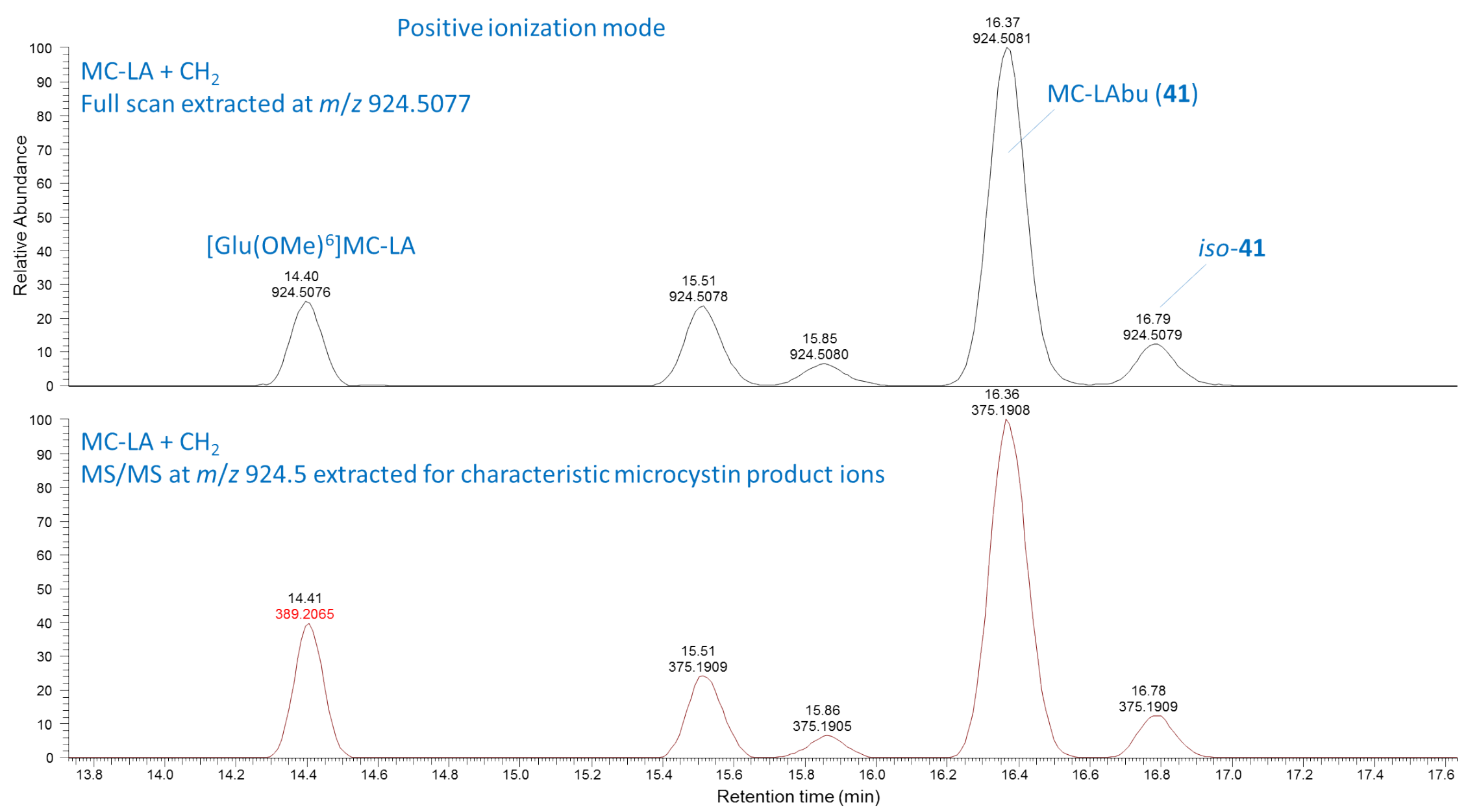

Figure S24. Top, full-scan positive mode LC-HRMS chromatograms extracted at the exact $[\mathrm{M}+\mathrm{H}]^{+} \mathrm{m} / z$ for MC-LAbu (41), and; bottom, LC-MS PRM chromatogram at $\mathrm{m} / \mathrm{z} 924.5$ extracted for $\mathrm{m} / \mathrm{z}$ of the characteristic microcystin product ions. 


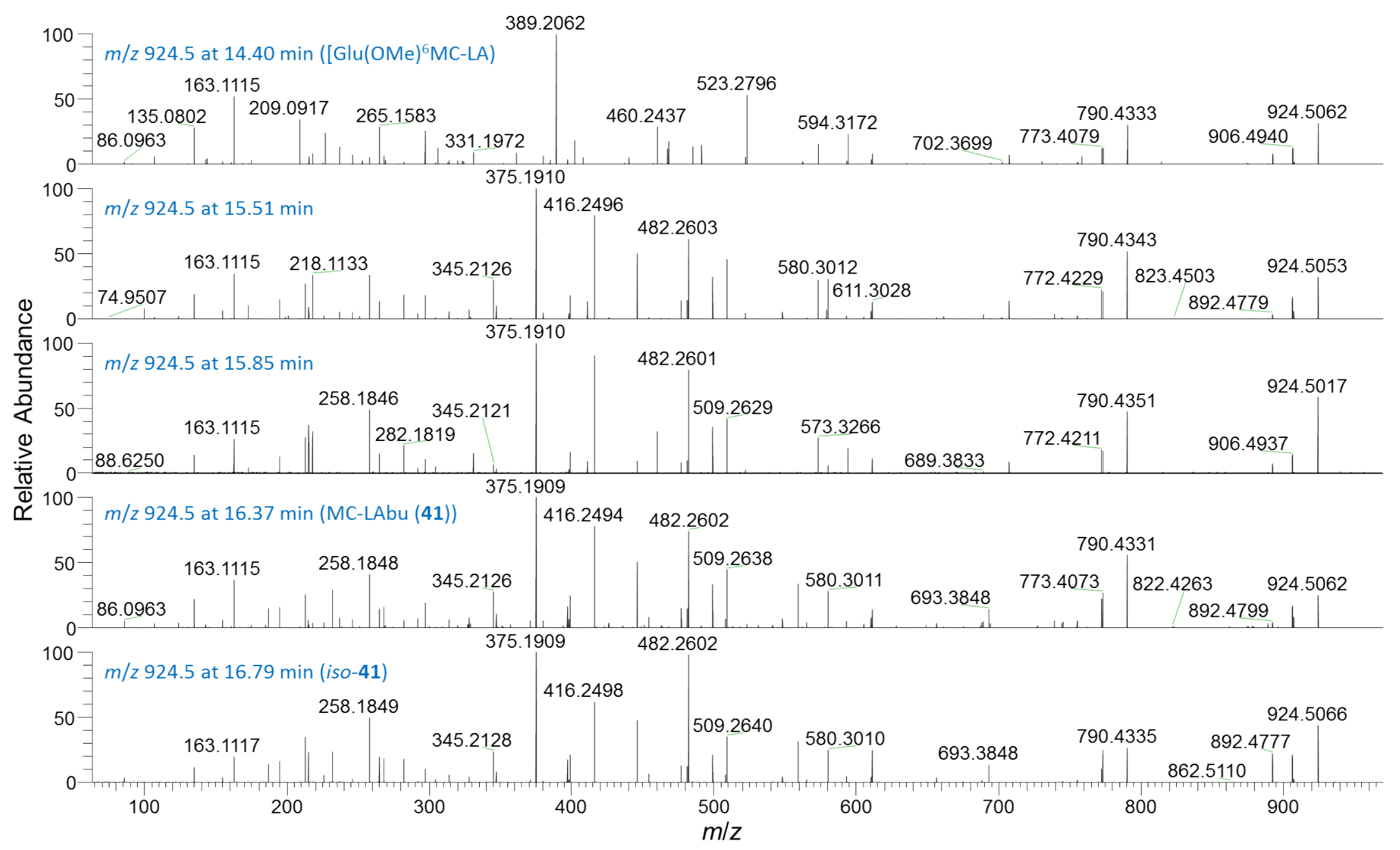

Figure S25. LC-MS PRM MS/MS spectra of $[\mathrm{M}+\mathrm{H}]^{+}$obtained at $m / z 924.5$ for the five peaks shown in Figure S24. 


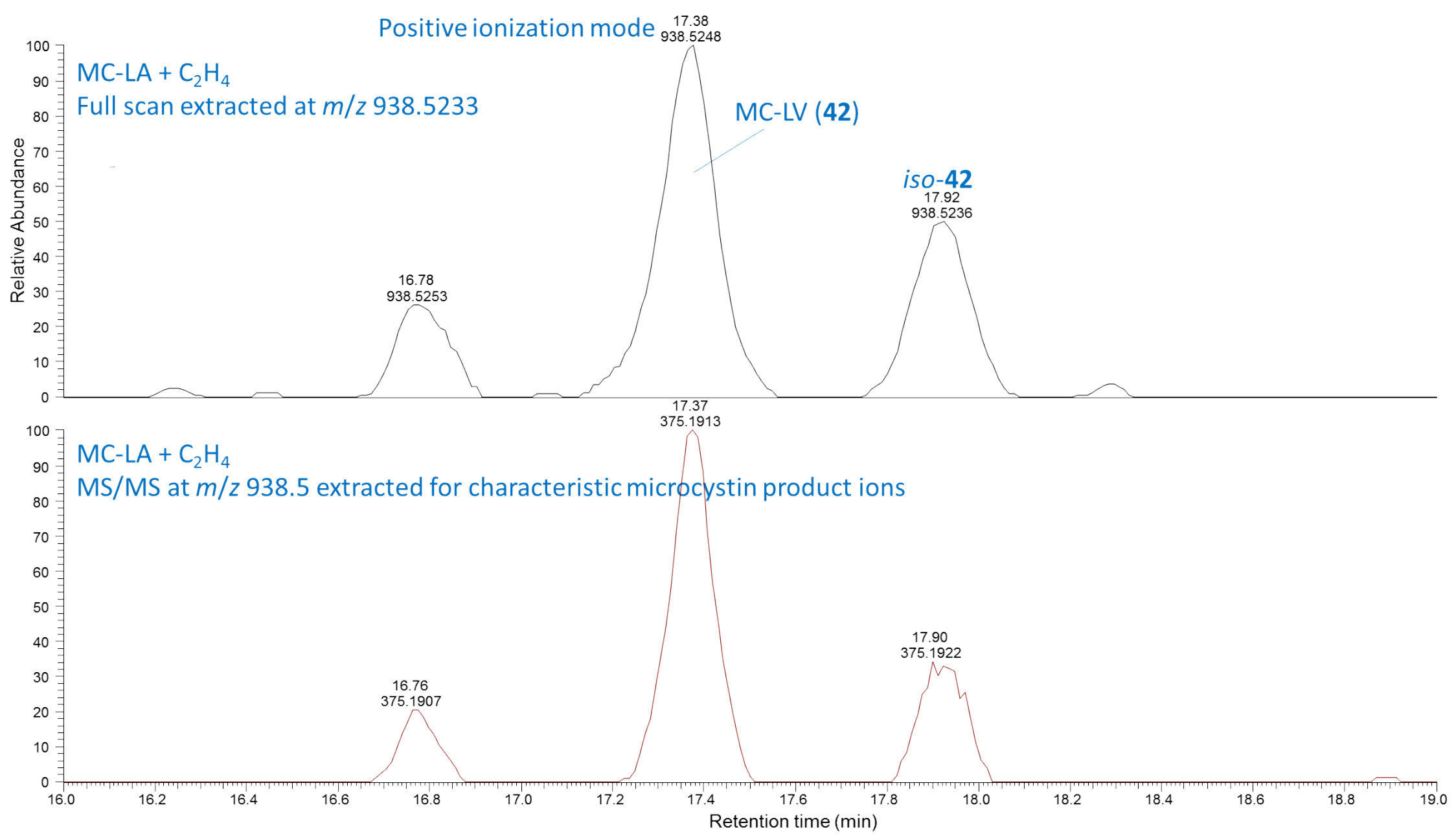

Figure S26. Top, full-scan positive mode LC-HRMS chromatograms extracted at the exact $[\mathrm{M}+\mathrm{H}]^{+} m / z$ for MC-LV (42), and; bottom, LC-MS PRM chromatogram at $\mathrm{m} / \mathrm{z} 938.5$ extracted for $\mathrm{m} / \mathrm{z}$ of the characteristic microcystin product ions. 


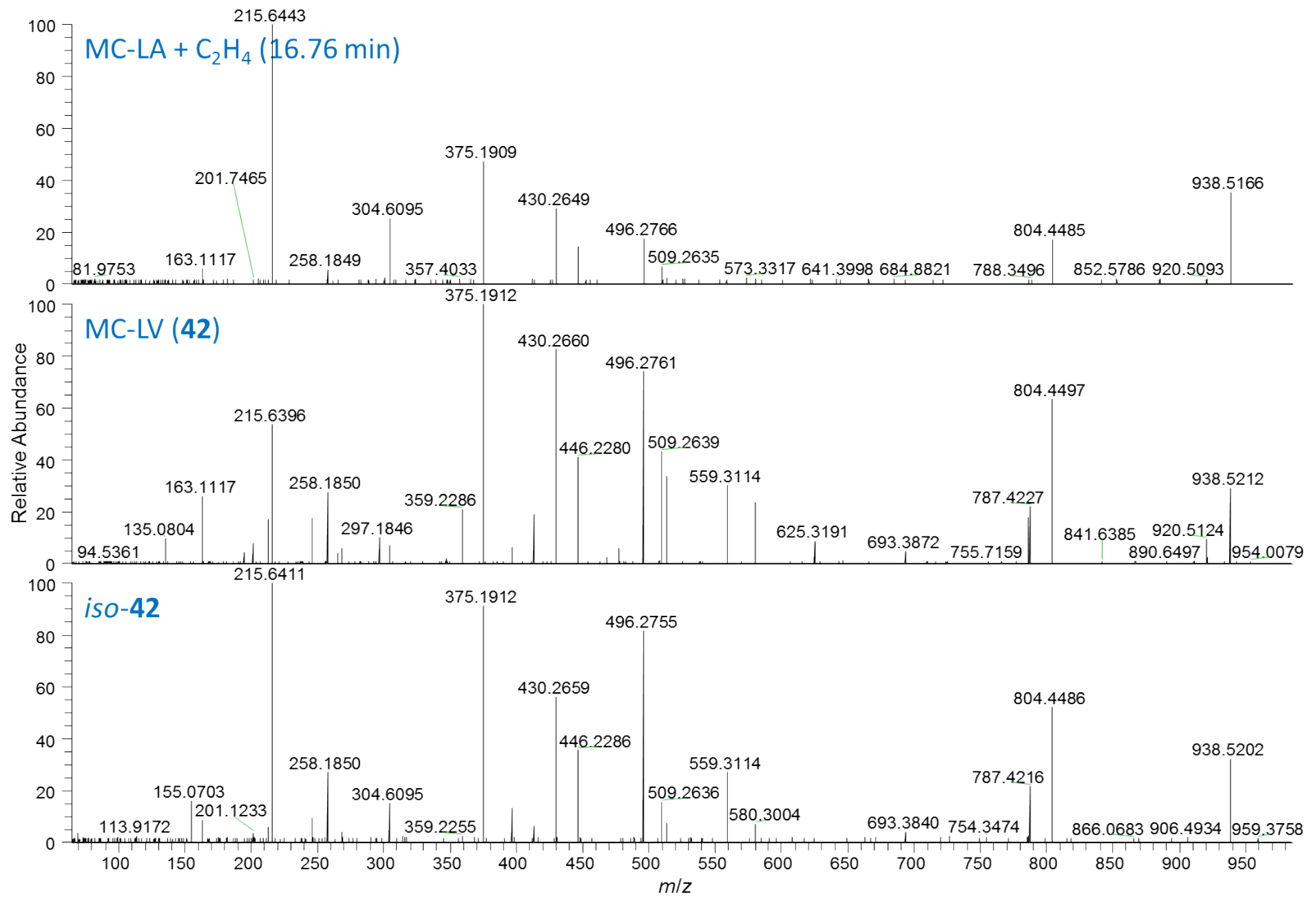

Figure S27. LC-MS PRM MS/MS spectra of $[\mathrm{M}+\mathrm{H}]^{+}$obtained in positive ionization mode at $\mathrm{m} / z$ 938.5 for the three peaks shown in Figure S26. 


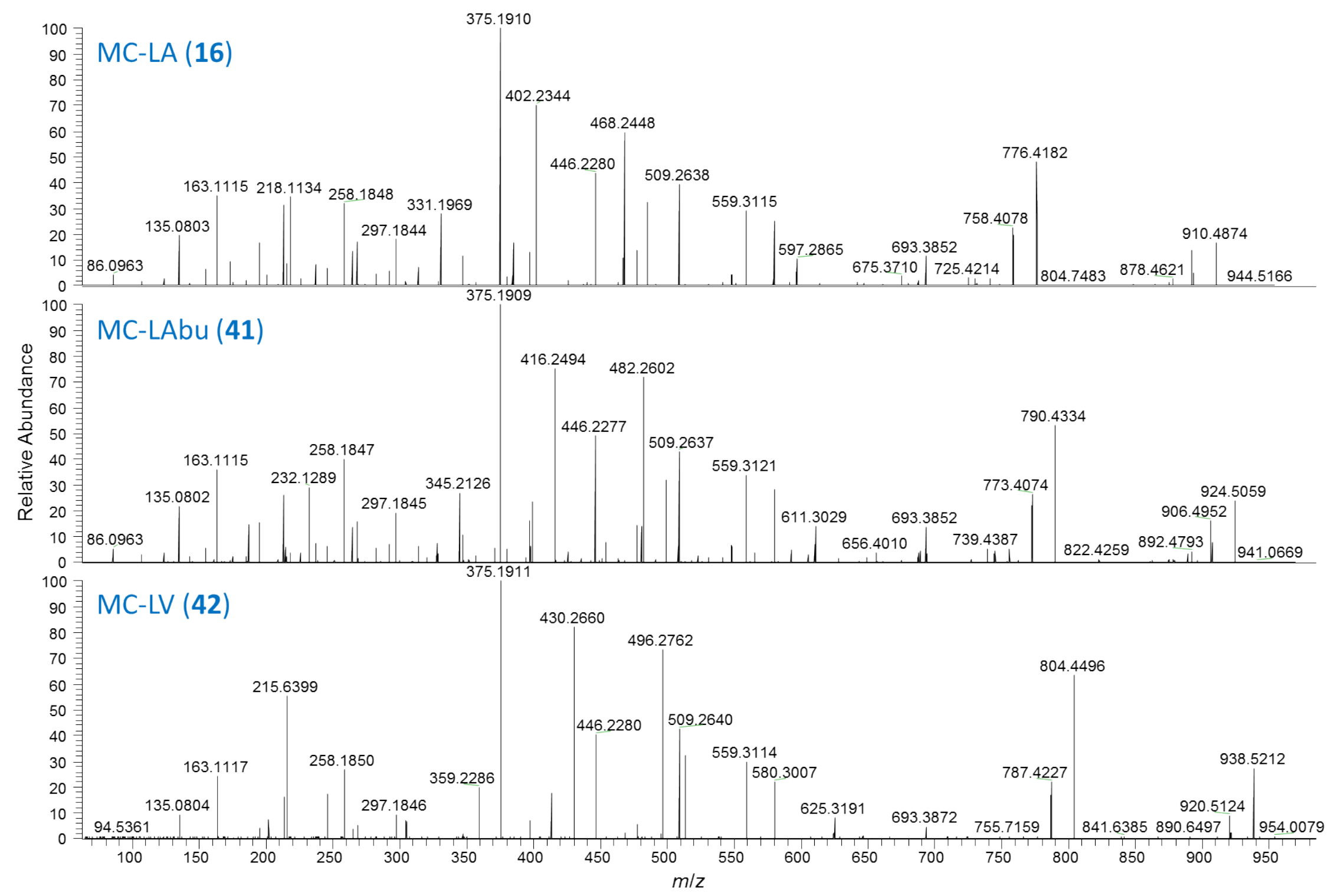

Figure S28. LC-MS PRM MS/MS spectra obtained in positive ionization mode for $[\mathrm{M}+\mathrm{H}]^{+}$of MC-LA (16), MC-LAbu (41) and MCLV (42). 


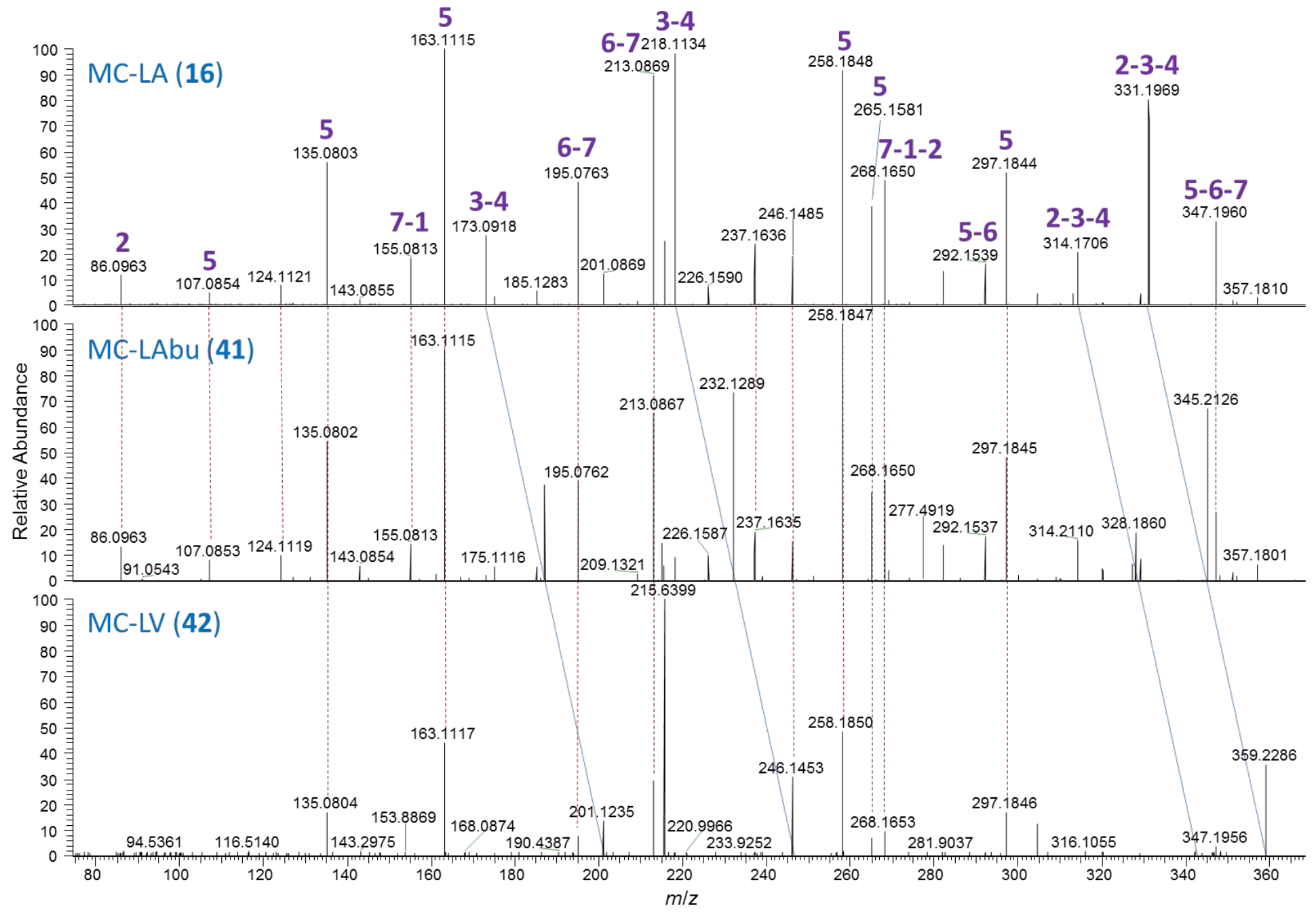

Figure 29. Expansion of LC-MS PRM MS/MS spectra obtained in positive ionization mode for $[\mathrm{M}+\mathrm{H}]^{+}$of MC-LA (16), MC-LAbu (41) and MC-LV (42). Blue lines connect ions that increase relative to MC-LA by 14.0157 in 41 and by 28.0313 in 42 (corresponding to $\mathrm{CH}_{2}$, and $\mathrm{C}_{2} \mathrm{H}_{4}$, respectively), while dashed red lines connect ions that remain unchanged. Bold purple numbers indicate amino acid residues associated with selected product ions in $\mathbf{1 6}$. Mass differences in product ions are seen only with changes in the amino acid at position-4. 


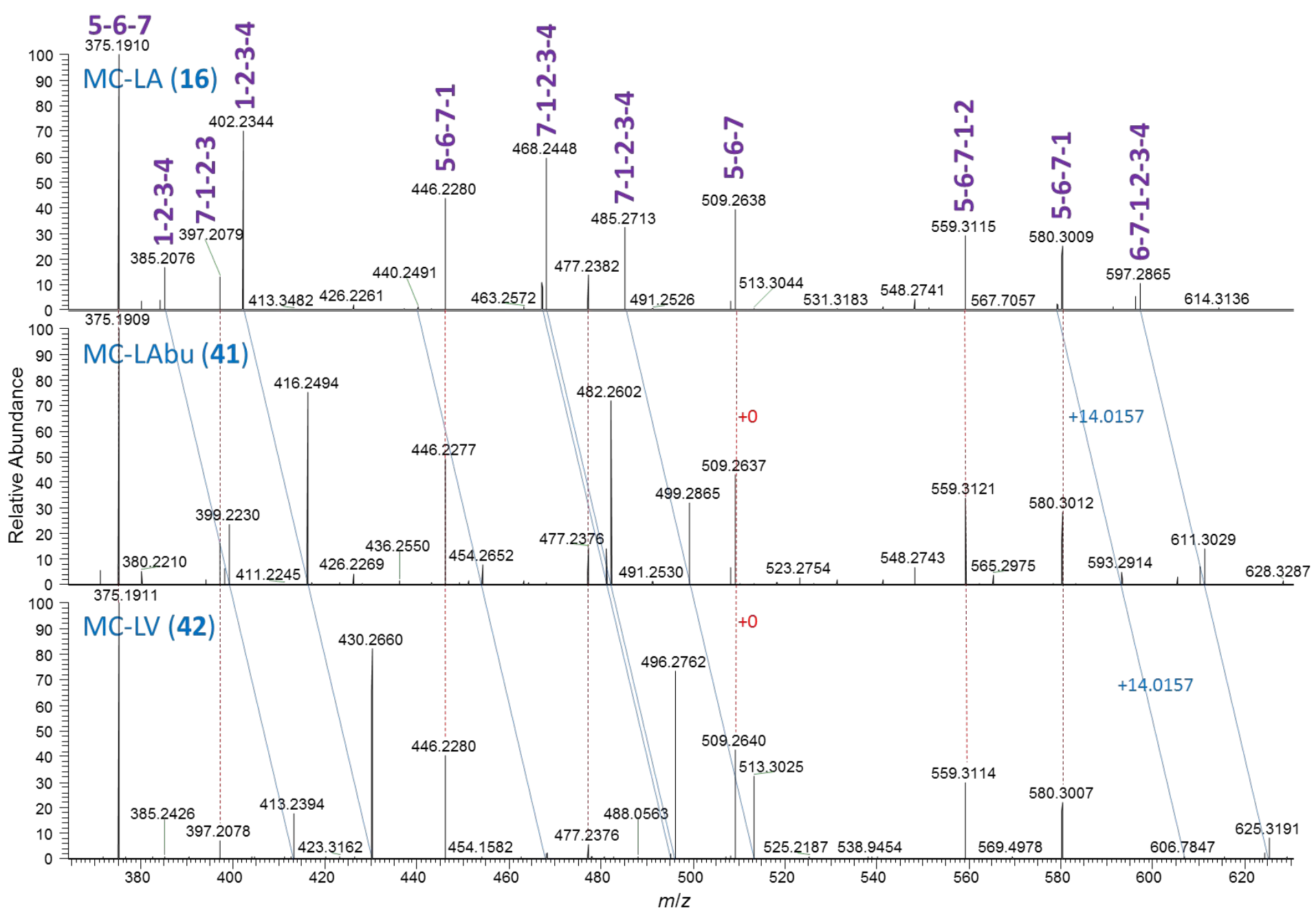

Figure S30. Expansion of LC-MS PRM MS/MS spectra obtained in positive ionization mode for [M+H] ${ }^{+}$of MC-LA (16), MC-LAbu (41) and MC-LV (42). Blue lines connect ions that increase relative to MC-LA by 14.0157 in 41 and by 28.0313 in 42 (corresponding to $\mathrm{CH}_{2}$, and $\mathrm{C}_{2} \mathrm{H}_{4}$, respectively), while dashed red lines connect ions that remain unchanged. Bold purple numbers indicate amino acid residues associated with selected product ions in 16. Mass differences in product ions are seen only with changes in the amino acid at position-4. 


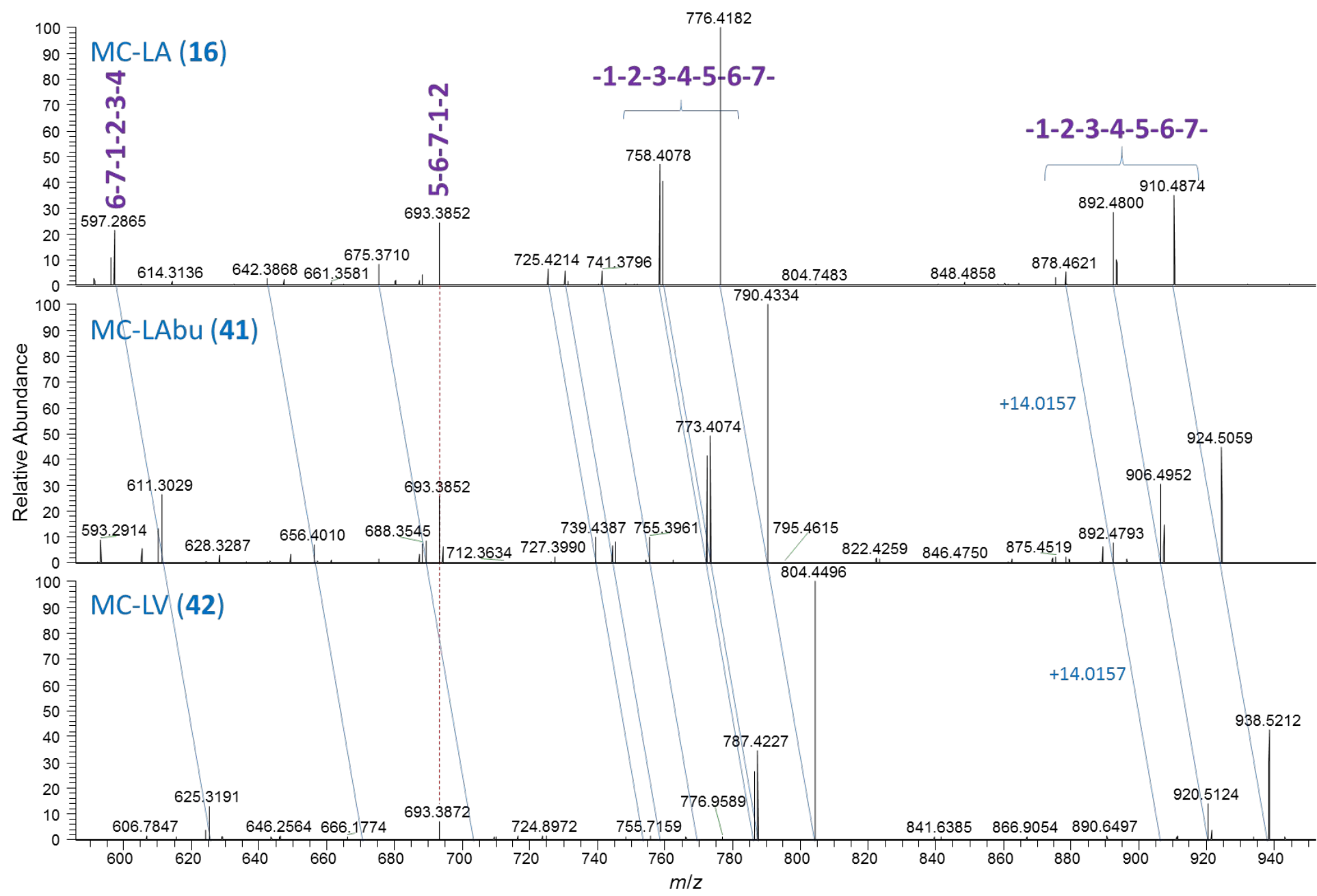

Figure S31. Expansion of LC-MS PRM MS/MS spectra obtained in positive ionization mode for [M+H] ${ }^{+}$of MC-LA (16), MC-LAbu (41) and MC-LV (42). Blue lines connect ions that increase relative to MC-LA by 14.0157 in 41 and by 28.0313 in 42 (corresponding to $\mathrm{CH}_{2}$, and $\mathrm{C}_{2} \mathrm{H}_{4}$, respectively), while dashed red lines connect ions that remain unchanged. Bold purple numbers indicate amino acid residues associated with selected product ions in 16. Mass differences in product ions are seen only with changes in the amino acid at position-4. 

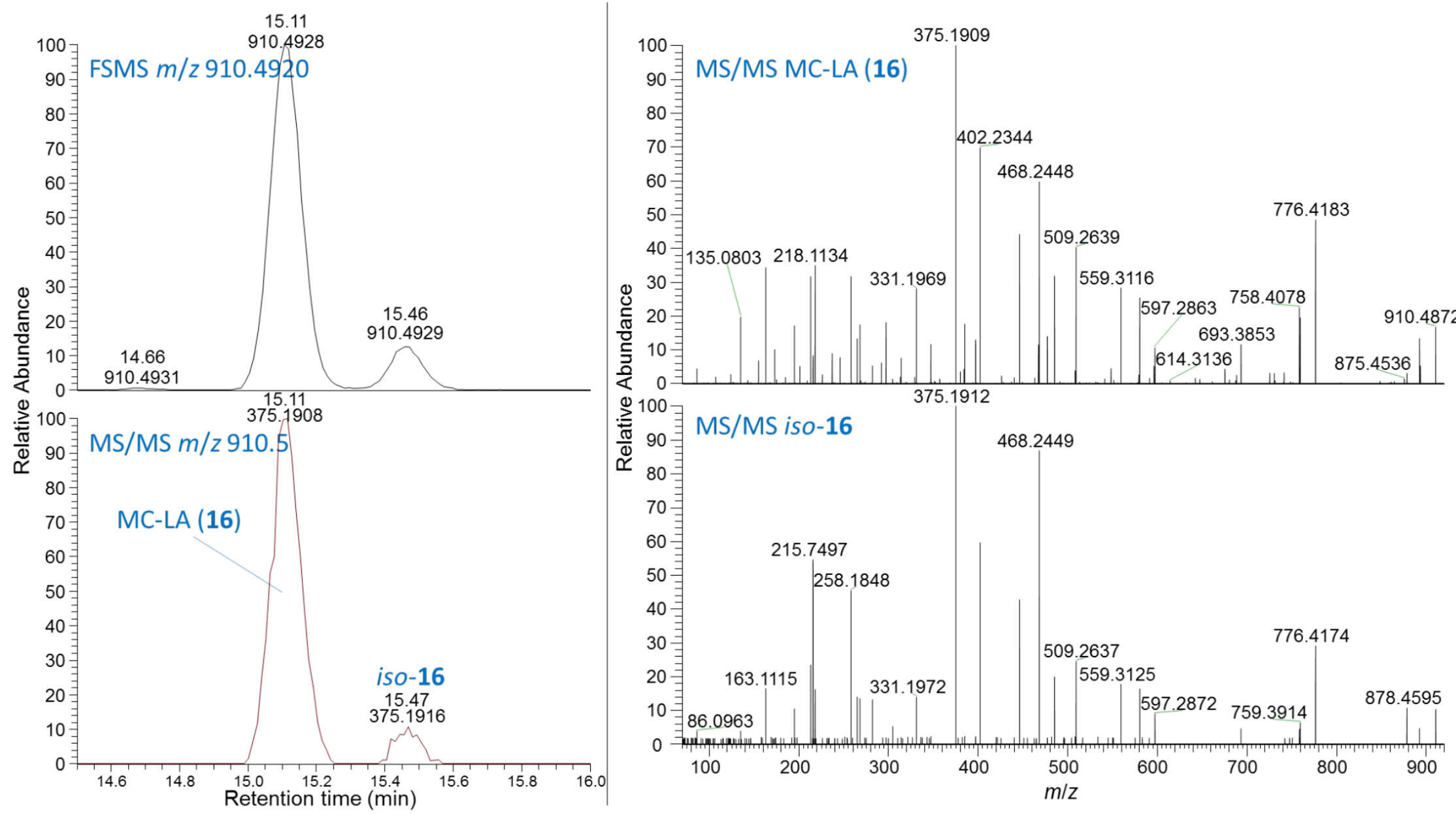

Figure 32. Top left, full-scan positive mode LC-HRMS chromatograms extracted at the exact $[\mathrm{M}+\mathrm{H}]^{+} \mathrm{m} / z$ for MC-LA (16), and; bottom left, LC-MS PRM chromatogram at $\mathrm{m} / z$ 910.5 extracted for $\mathrm{m} / z$ of the characteristic microcystin product ions. At right MS/MS spectra of MC-LA (16) and iso-16 extracted from the PRM chromatogram. 


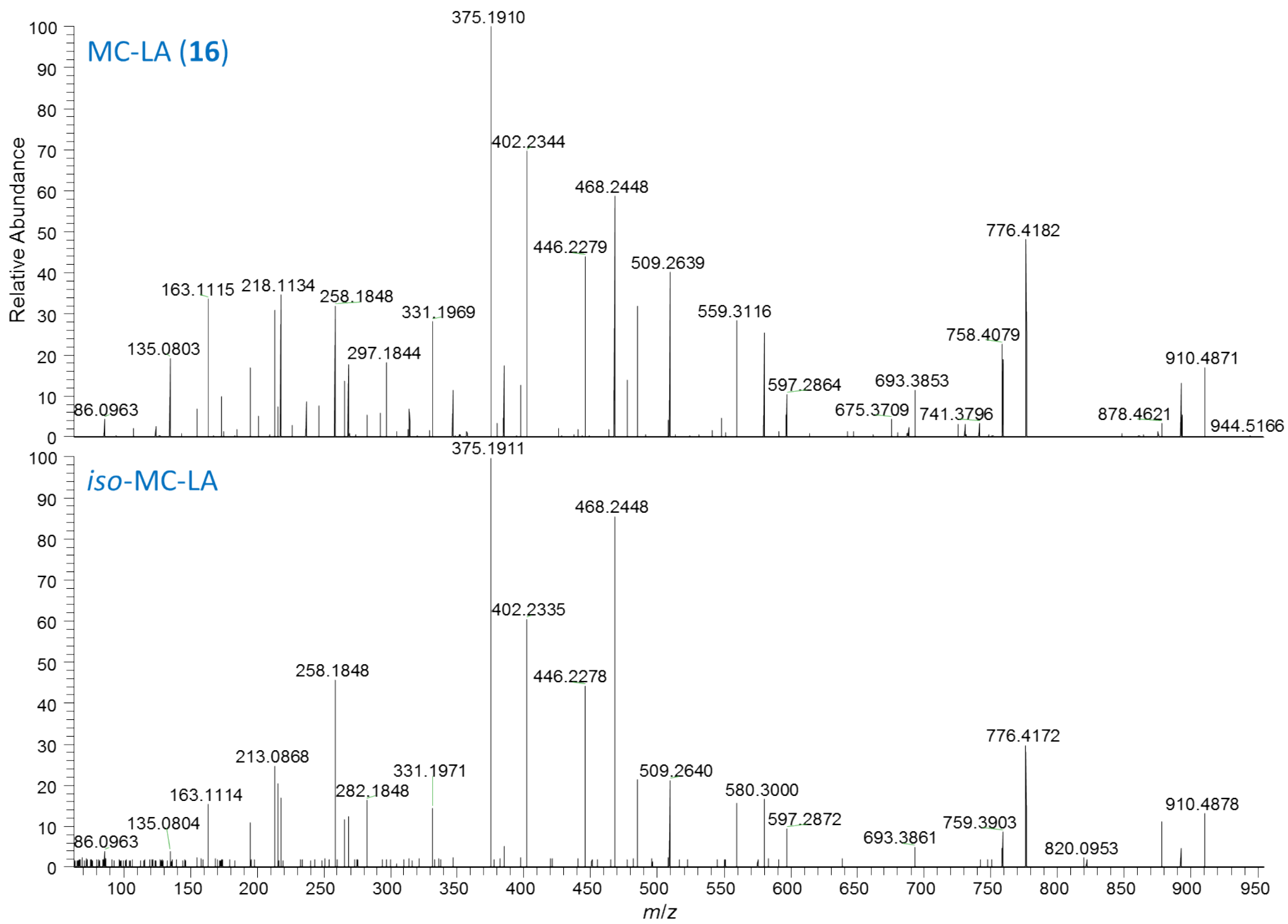

Figure S33. LC-MS PRM MS/MS spectra of $[\mathrm{M}+\mathrm{H}]^{+}$obtained at $m / z 910.5$ for the two peaks shown in Figure S32. 


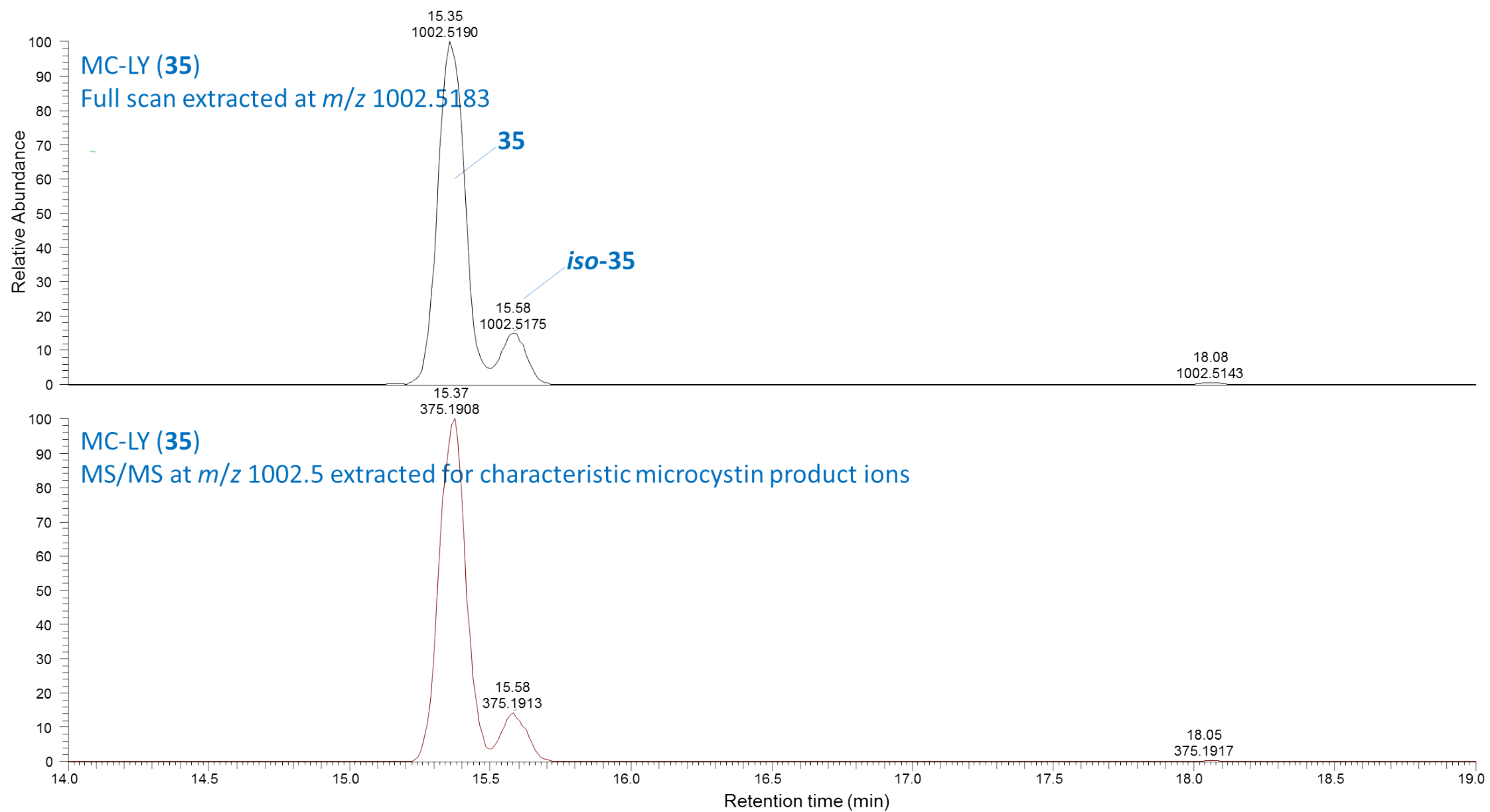

Figure S34. Top, full-scan positive mode LC-HRMS chromatograms extracted at the exact $[\mathrm{M}+\mathrm{H}]^{+} m / z$ for MC-LY (35), and; bottom, LC-MS PRM chromatogram at $\mathrm{m} / \mathrm{z} 1002.5$ extracted for $\mathrm{m} / \mathrm{z}$ of the characteristic microcystin product ions. 


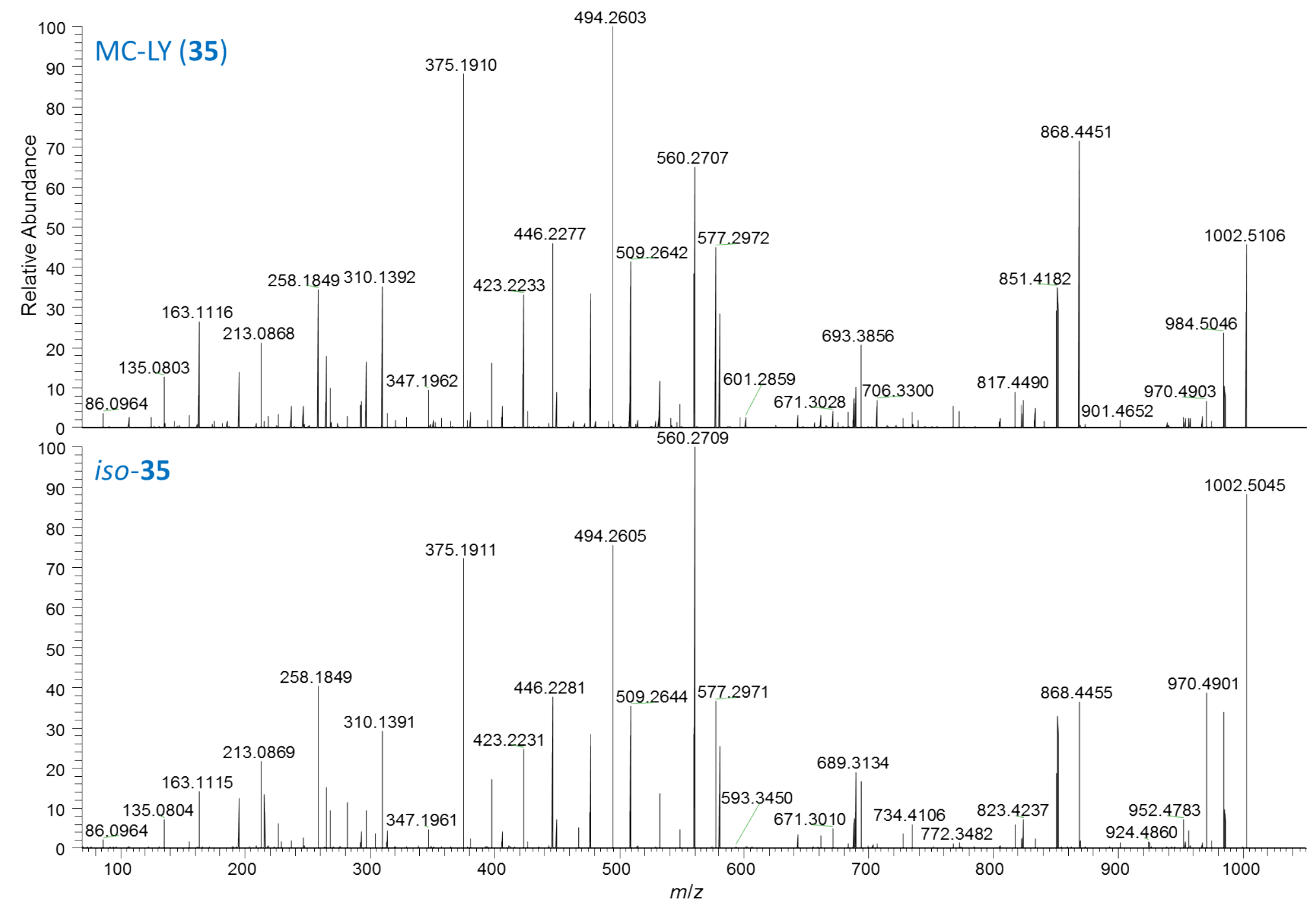

Figure S35. LC-MS PRM MS/MS spectra of $[\mathrm{M}+\mathrm{H}]^{+}$obtained at $m / z 1002.5$ for the two peaks shown in Figure S34. 


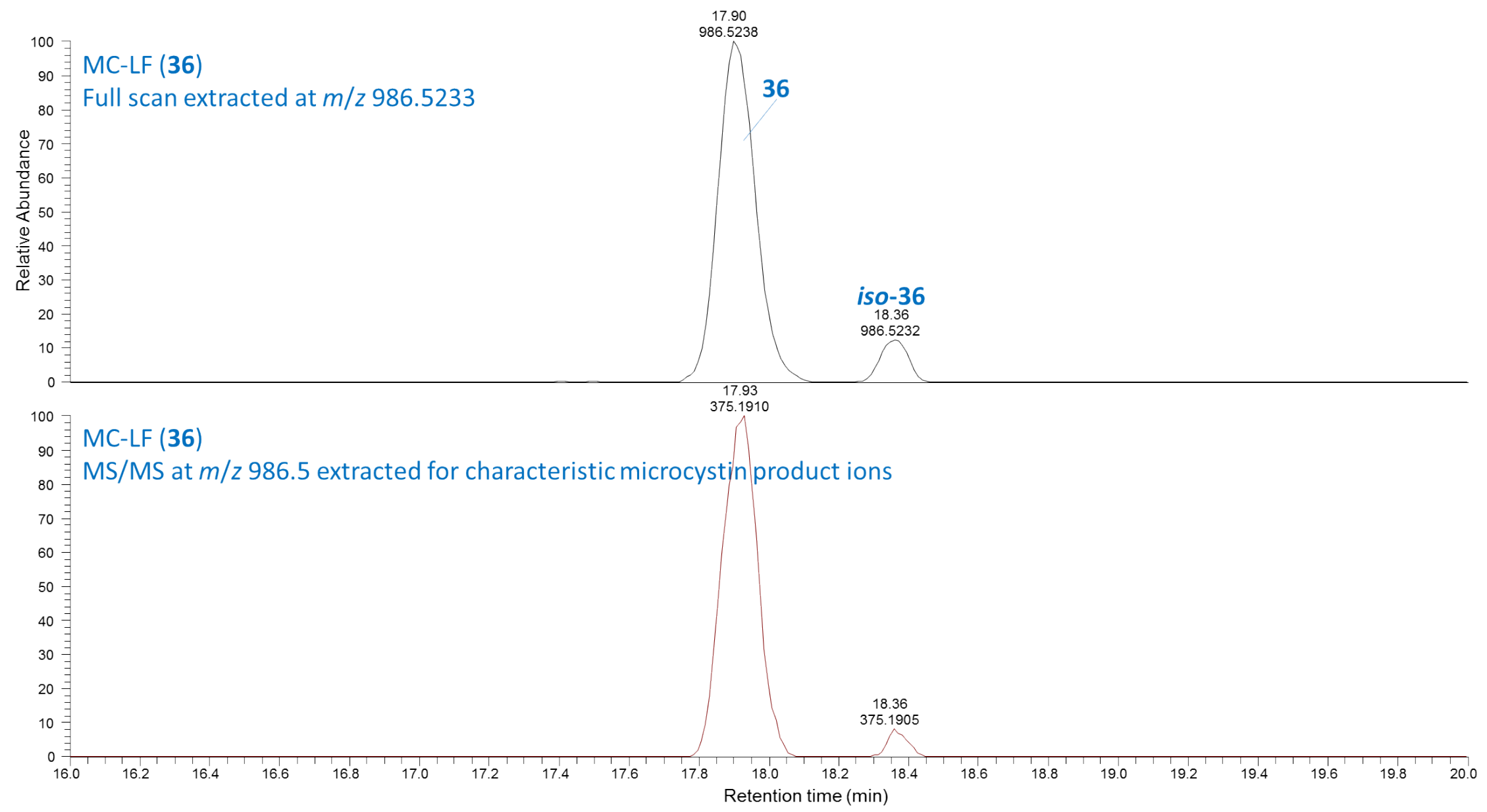

Figure S36. Top, full-scan positive mode LC-HRMS chromatograms extracted at the exact $[\mathrm{M}+\mathrm{H}]^{+} \mathrm{m} / z$ for MC-LF (36), and; bottom, LC-MS PRM chromatogram at $\mathrm{m} / \mathrm{z} 986.5$ extracted for $\mathrm{m} / \mathrm{z}$ of the characteristic microcystin product ions. 


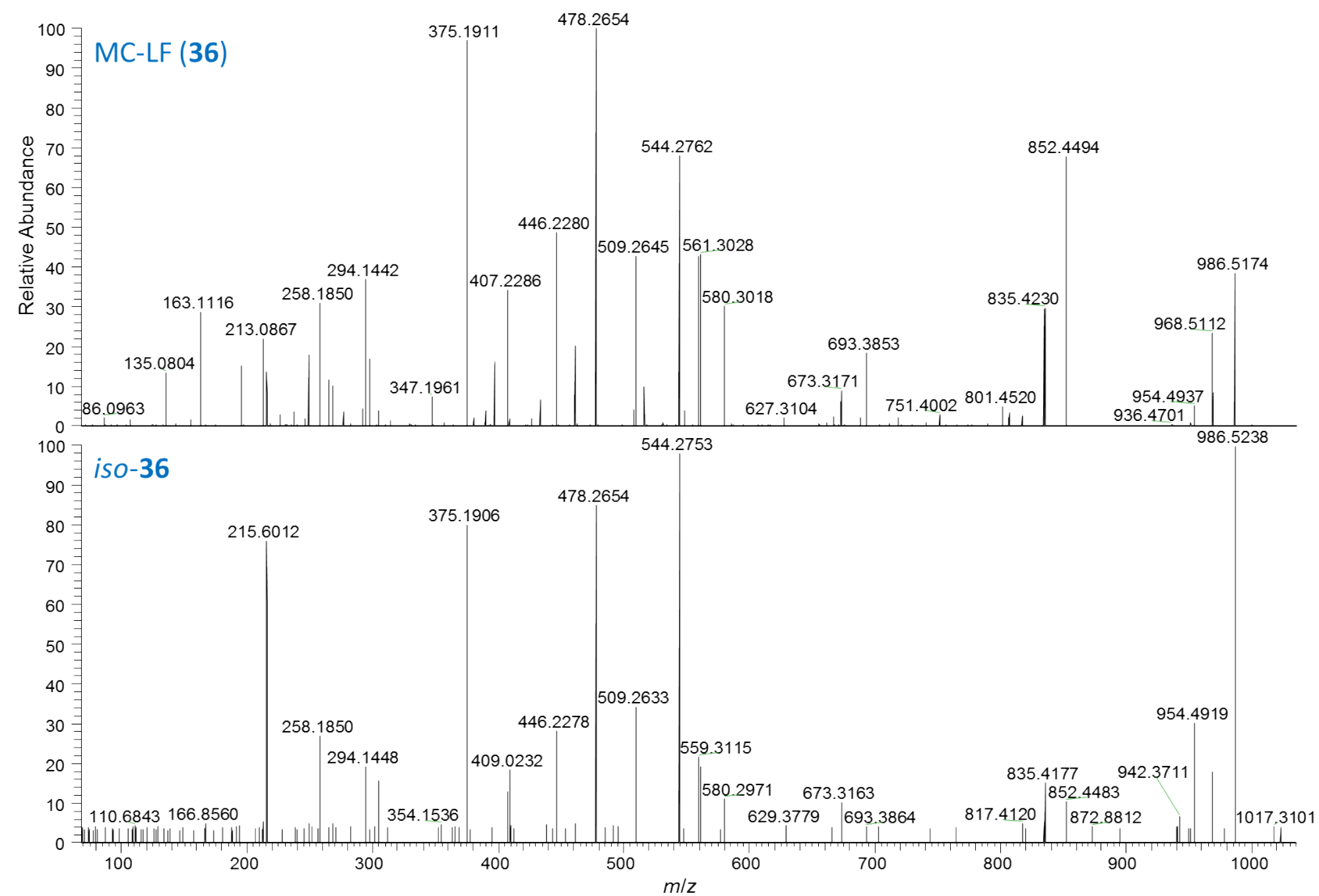

Figure S37. LC-MS PRM MS/MS spectra of $[\mathrm{M}+\mathrm{H}]^{+}$obtained at $m / z 986.5$ for the two peaks shown in Figure S36. 


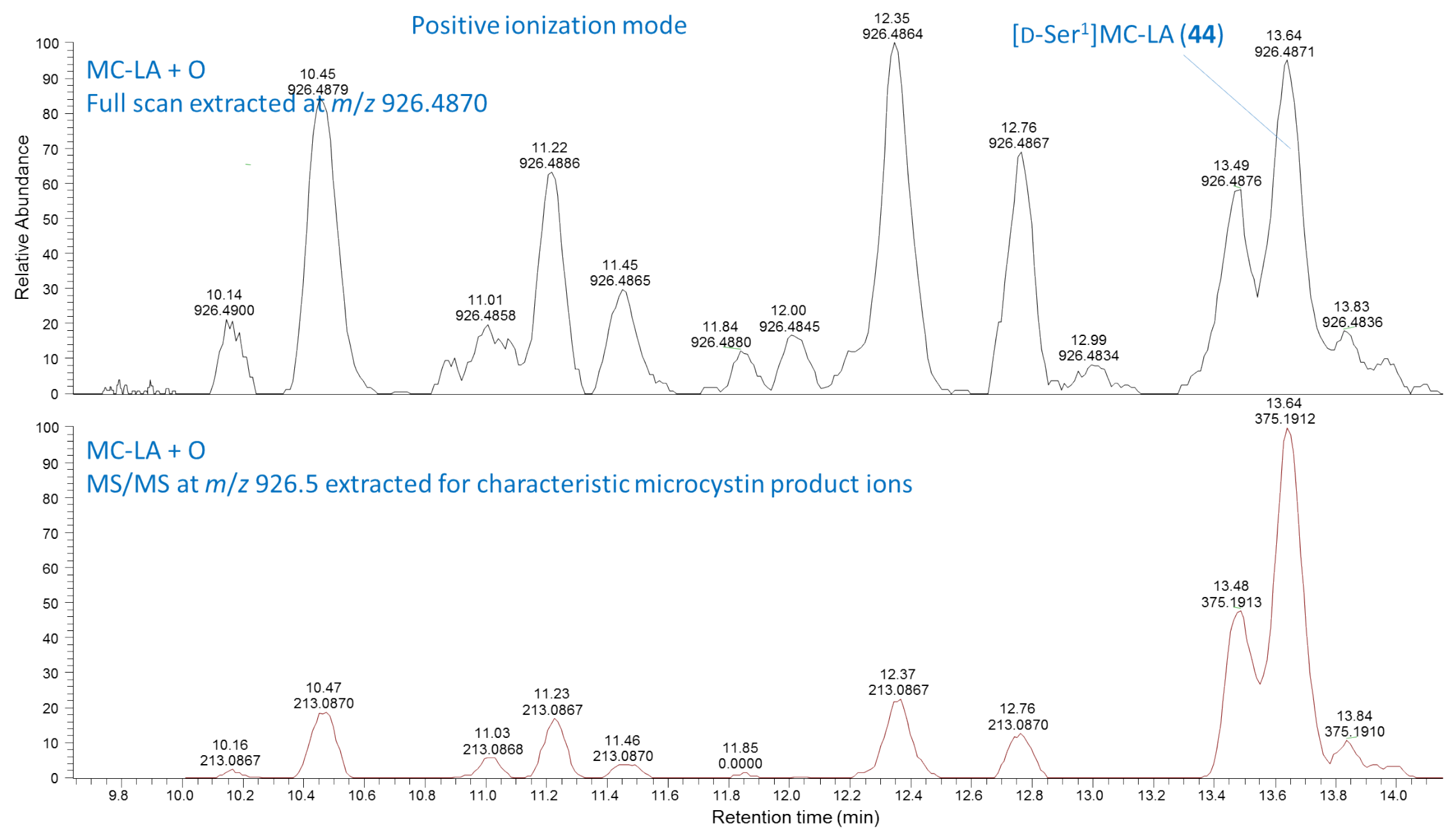

Figure S38. Top, full-scan positive mode LC-HRMS chromatograms extracted at the exact $[\mathrm{M}+\mathrm{H}]^{+} m / z$ for $\mathrm{MC}$-LA + oxygen, and; bottom, LC-MS PRM chromatogram at $\mathrm{m} / \mathrm{z} 926.5$ extracted for $\mathrm{m} / \mathrm{z}$ of the characteristic microcystin product ions. The peak identified as belonging to [D-Ser ${ }^{1}$ MC-LA (44) is marked. 


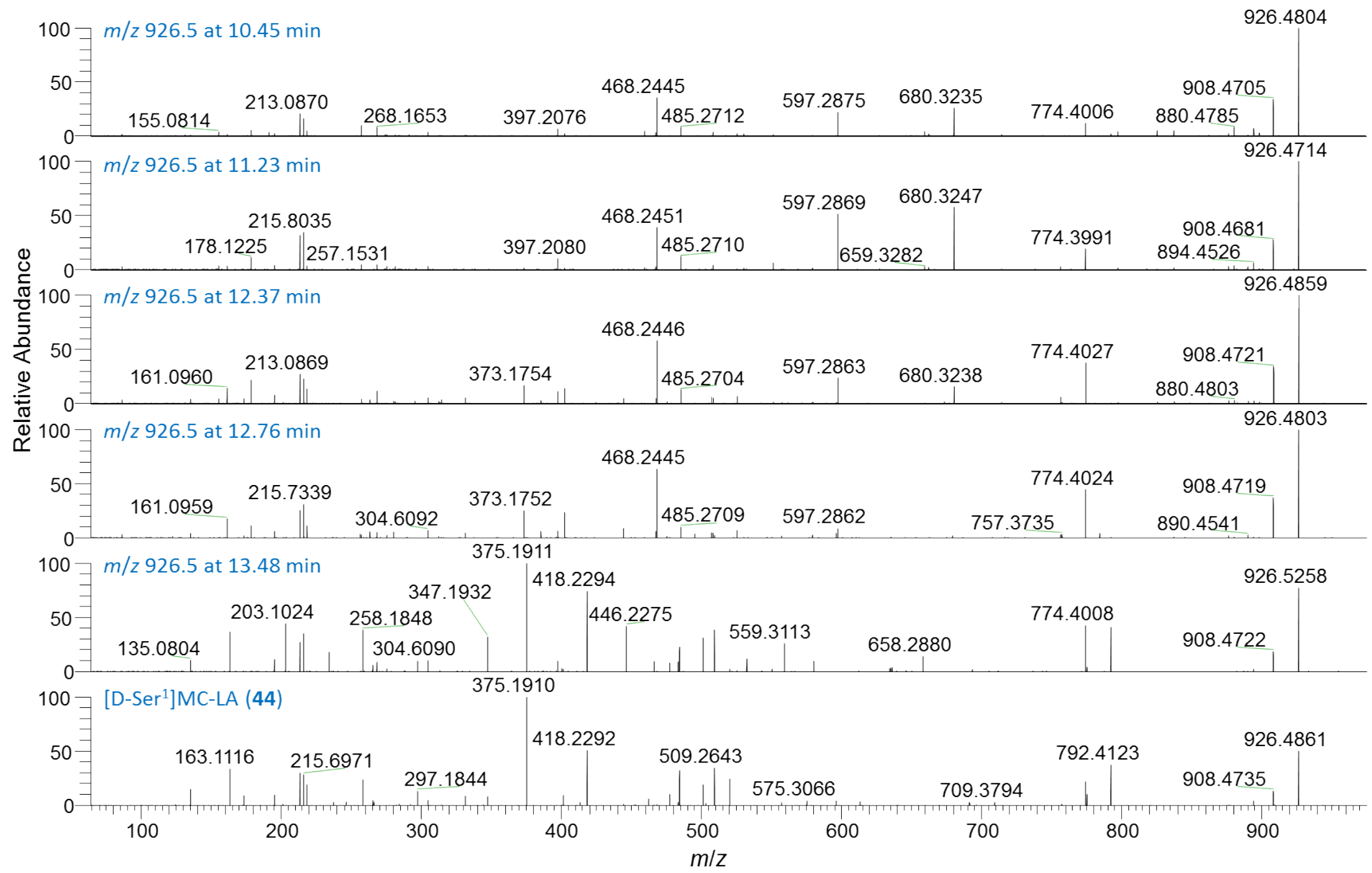

Figure S39. LC-MS PRM MS/MS spectra of $[\mathrm{M}+\mathrm{H}]^{+}$obtained at $m / z 926.5$ for the peaks shown in Figure S38. 


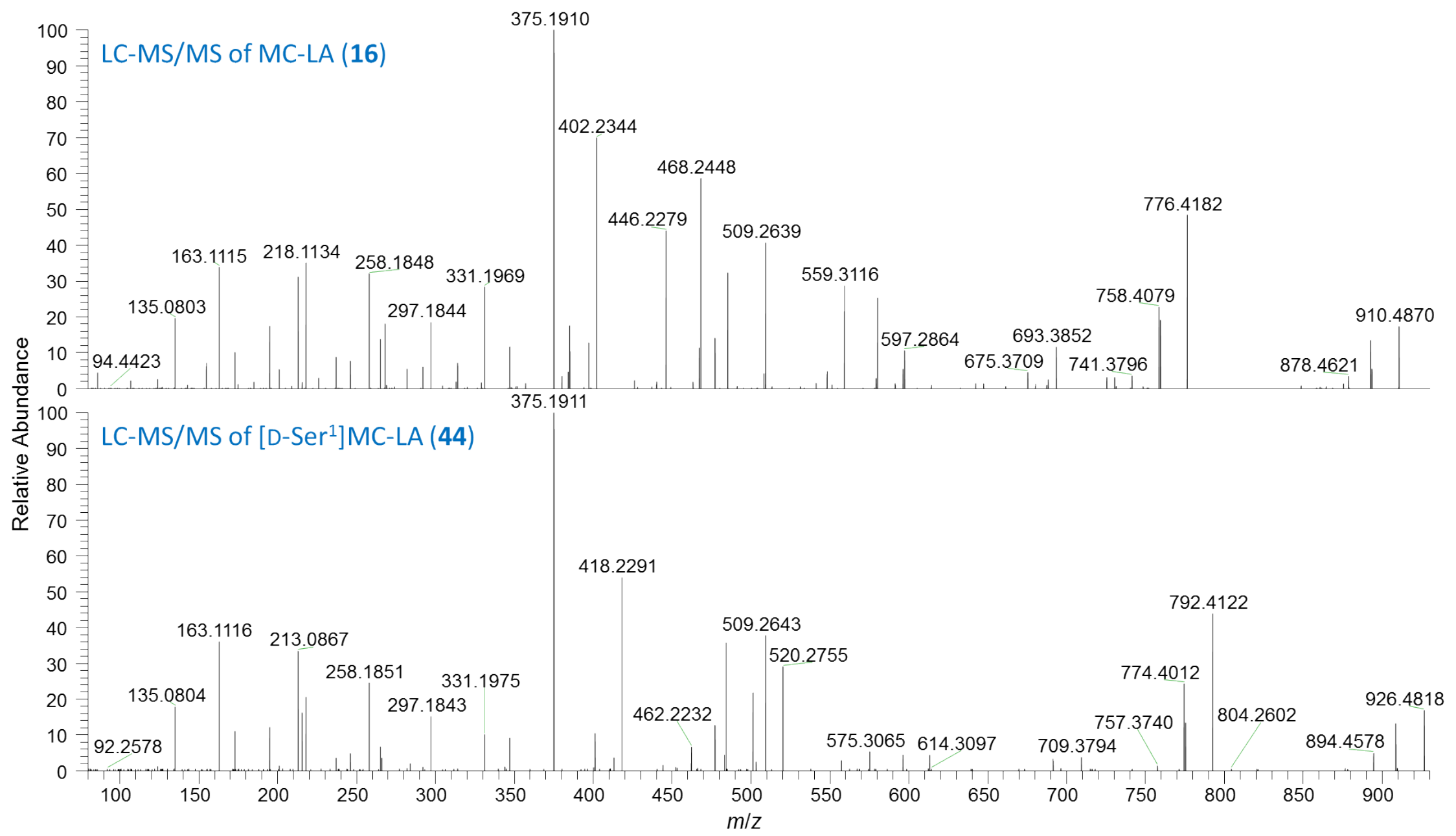

Figure 40. LC-MS PRM MS/MS spectra obtained in positive ionization mode for $[\mathrm{M}+\mathrm{H}]^{+}$of MC-LA (16) and [Ser $\left.{ }^{1}\right] \mathrm{MC}-\mathrm{LA}(\mathbf{4 4})$. 


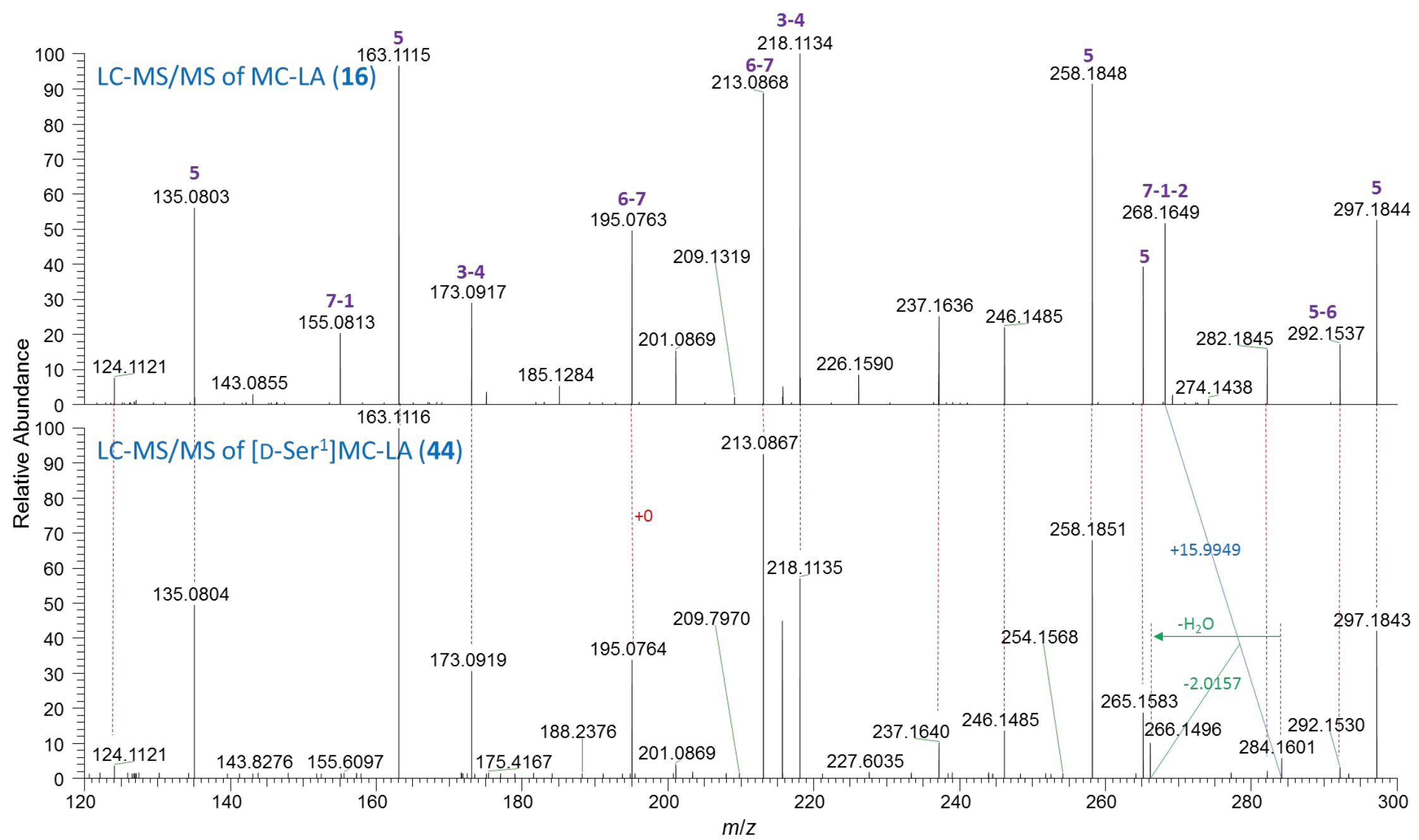

Figure S41. Expansion of LC-MS PRM MS/MS spectra obtained in positive ionization mode for $[\mathrm{M}+\mathrm{H}]^{+}$of MC-LA (16) and [Ser ${ }^{1}$ MC-LA (44). Blue lines connect ions that increase relative to MC-LR by 15.9949 in 44 (corresponding to O), and green lines by show partial water losses from product ions containing Ser at position-1, while dashed red lines connect ions that remain unchanged. Bold purple numbers indicate amino acid residues associated with selected product ions in $\mathbf{1}$. Mass differences in product ions of $\mathbf{1 6}$ and $\mathbf{4 4}$ are seen only with product ions containing the amino acid at position-1. 


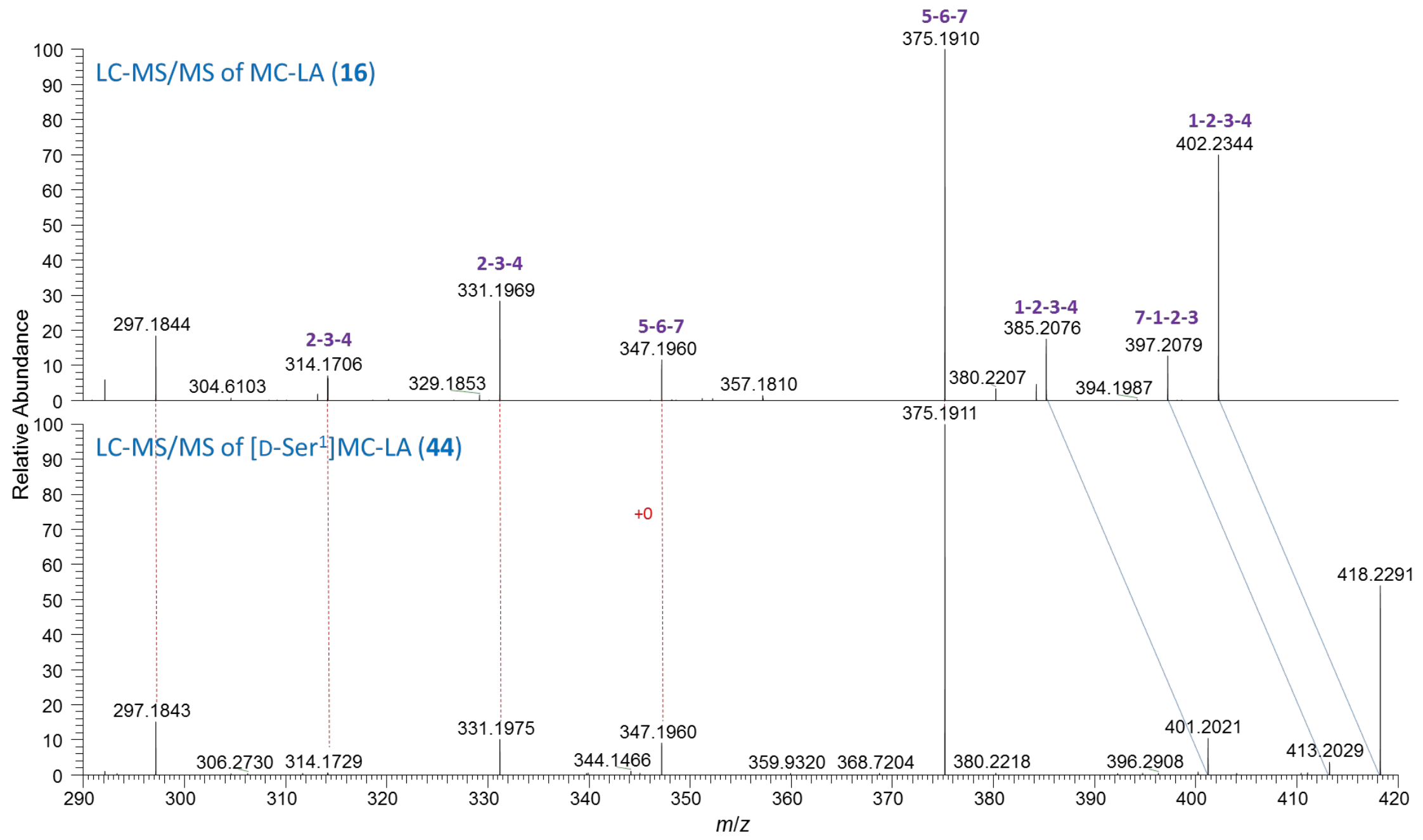

Figure S42. Expansion of LC-MS PRM MS/MS spectra obtained in positive ionization mode for [M+H] ${ }^{+}$of MC-LA (16) and [Ser ${ }^{1}$ ]MC-LA (44). Blue lines connect ions that increase relative to MC-LR by 15.9949 in 44 (corresponding to O), and green lines by show partial water losses from product ions containing Ser at position-1, while dashed red lines connect ions that remain unchanged. Bold purple numbers indicate amino acid residues associated with selected product ions in $\mathbf{1}$. Mass differences in product ions of $\mathbf{1 6}$ and $\mathbf{4 4}$ are seen only with product ions containing the amino acid at position-1. 


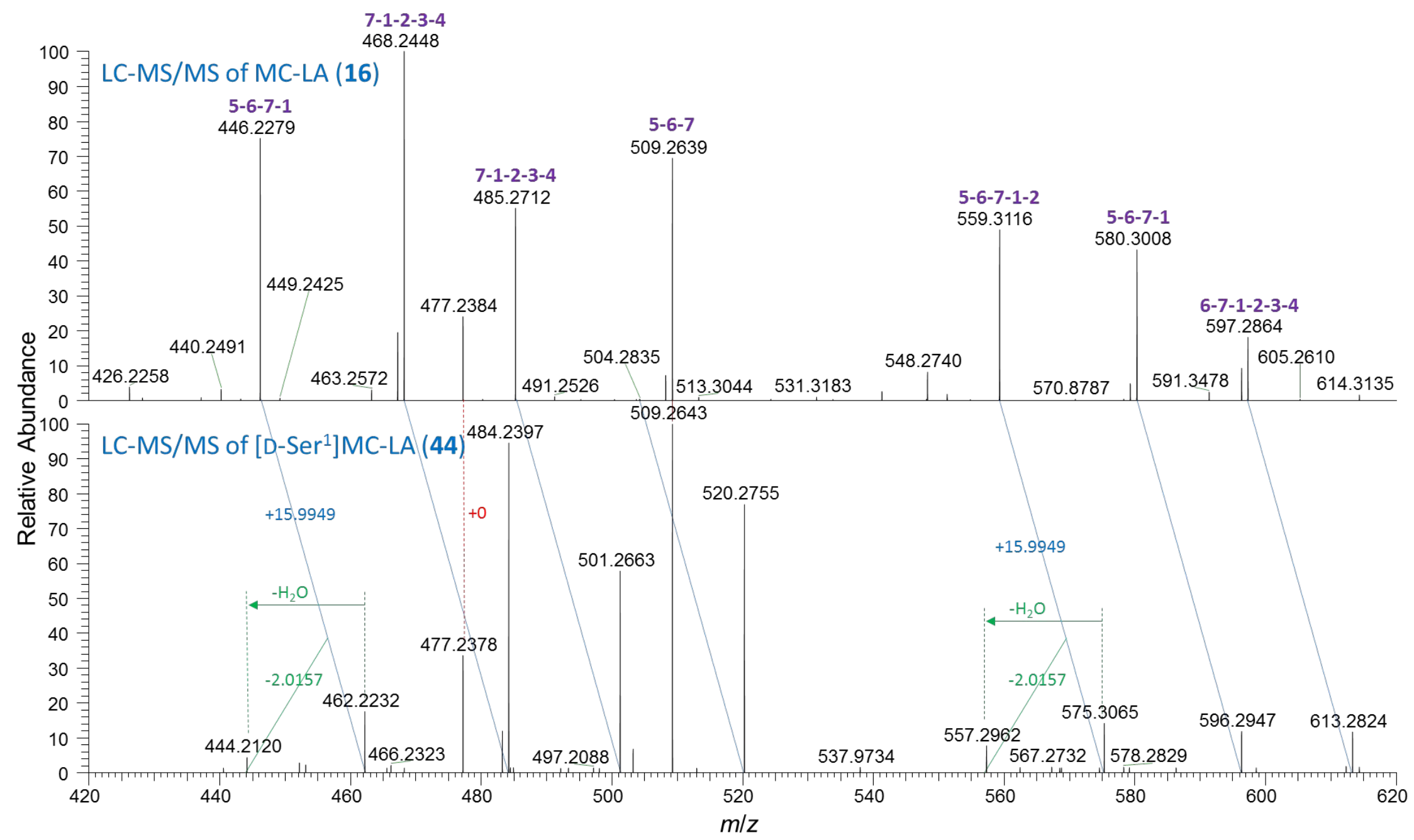

Figure S43. Expansion of LC-MS PRM MS/MS spectra obtained in positive ionization mode for $[\mathrm{M}+\mathrm{H}]^{+}$of MC-LA (16) and [Ser ${ }^{1}$ MC-LA (44). Blue lines connect ions that increase relative to MC-LR by 15.9949 in 44 (corresponding to O), and green lines by show partial water losses from product ions containing Ser at position-1, while dashed red lines connect ions that remain unchanged. Bold purple numbers indicate amino acid residues associated with selected product ions in $\mathbf{1}$. Mass differences in product ions of $\mathbf{1 6}$ and $\mathbf{4 4}$ are seen only with product ions containing the amino acid at position-1. 
$-1-2-3-4-5-6-7-$

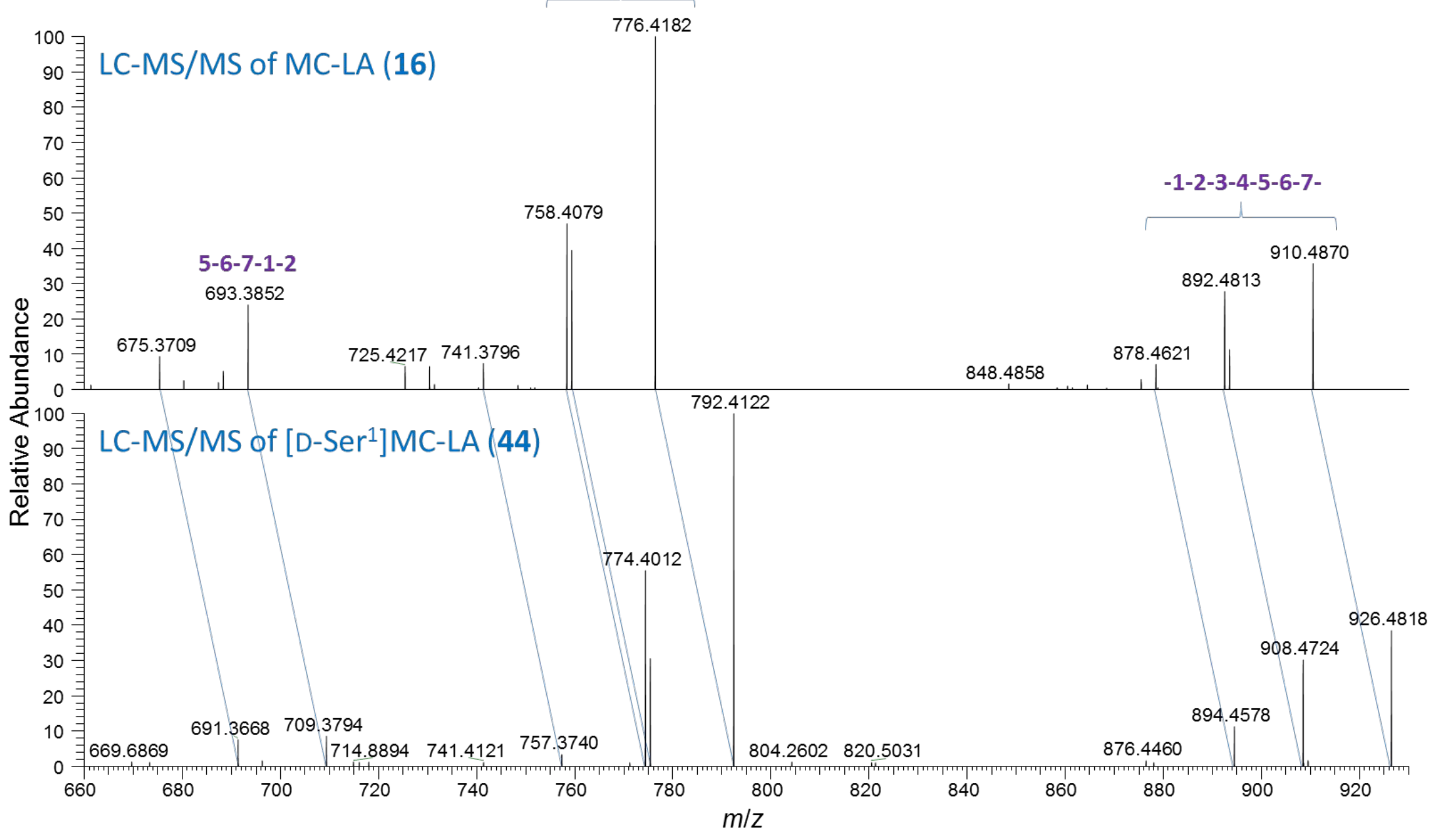

Figure S44. Expansion of LC-MS PRM MS/MS spectra obtained in positive ionization mode for $[\mathrm{M}+\mathrm{H}]^{+}$of MC-LA (16) and [Ser ${ }^{1}$ ]MC-LA (44). Blue lines connect ions that increase relative to MC-LR by 15.9949 in 44 (corresponding to O), and green lines by show partial water losses from product ions containing Ser at position-1, while dashed red lines connect ions that remain unchanged. Bold purple numbers indicate amino acid residues associated with selected product ions in $\mathbf{1}$. Mass differences in product ions of $\mathbf{1 6}$ and $\mathbf{4 4}$ are seen only with product ions containing the amino acid at position-1. 


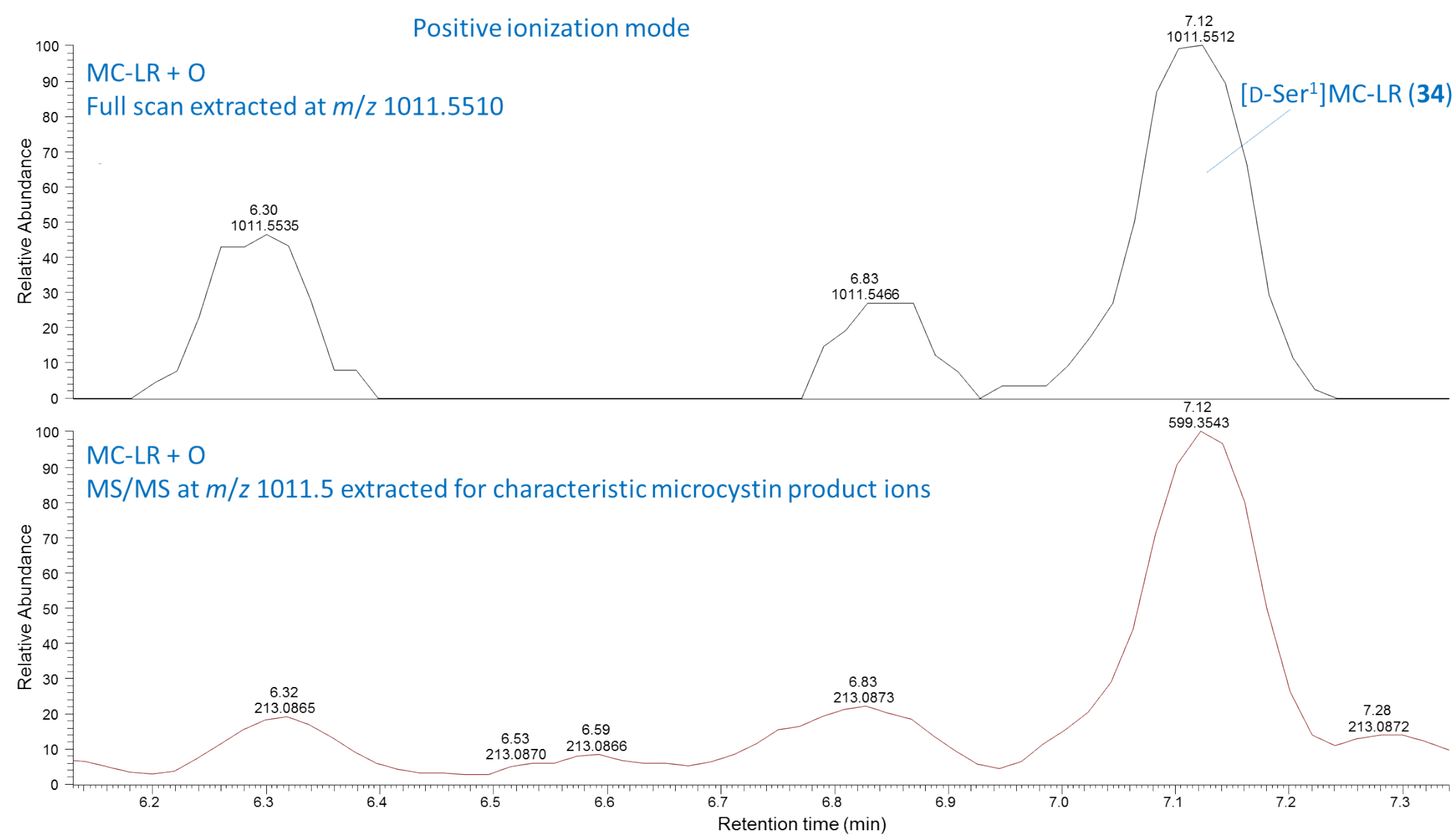

Figure S45. Top, full-scan positive mode LC-HRMS chromatograms extracted at the exact $[\mathrm{M}+\mathrm{H}]^{+} m / z$ for MC-LR + oxygen, and; bottom, LC-MS PRM chromatogram at $\mathrm{m} / \mathrm{z} 1011.5$ extracted for $\mathrm{m} / \mathrm{z}$ of the characteristic microcystin product ions. The peak identified as belonging to [D-Ser $\left.{ }^{1}\right] \mathrm{MC}-\mathrm{LR}(\mathbf{3 4})$ is marked. 

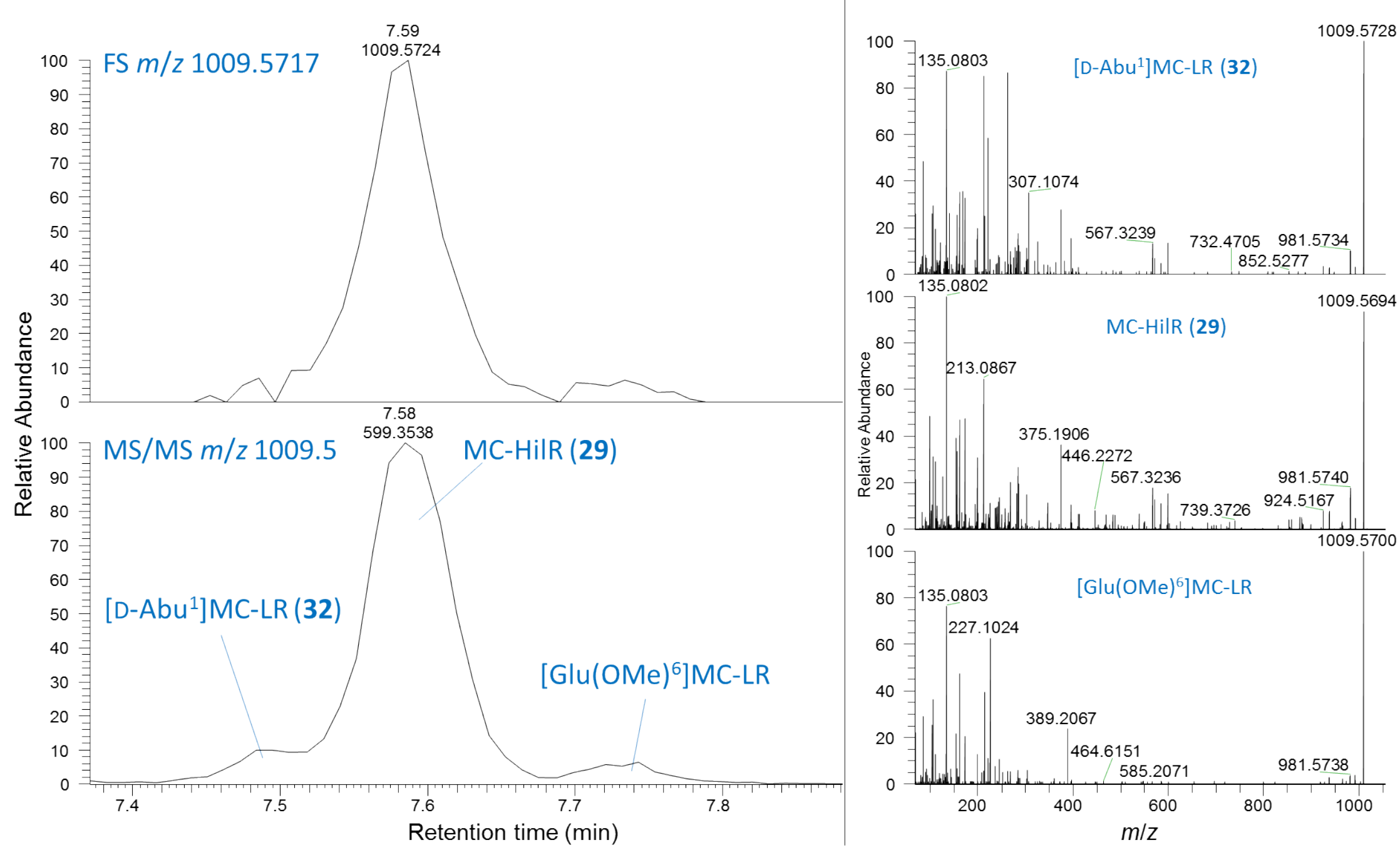

Figure S50. Top left, full-scan positive mode LC-HRMS chromatograms extracted at the exact $[\mathrm{M}+\mathrm{H}]^{+} m / z$ for $\mathrm{MC}-\mathrm{LR}+\mathrm{CH}{ }_{2}$, and; bottom left, LC-MS PRM chromatogram at $\mathrm{m} / z 1009.5$ extracted for $\mathrm{m} / z$ of the characteristic microcystin product ions. Peaks identified as as $\left[\mathrm{D}-\mathrm{Abu}^{1}\right] \mathrm{MC}-\mathrm{LR}(\mathbf{3 2}), \mathrm{MC}-\mathrm{HilR}(\mathbf{2 9})$ and $\left[\mathrm{Glu}(\mathrm{OMe})^{6}\right] \mathrm{MC}-\mathrm{LR}$ are indicated, and their corresponding MS/MS spectra are shown on the right. 


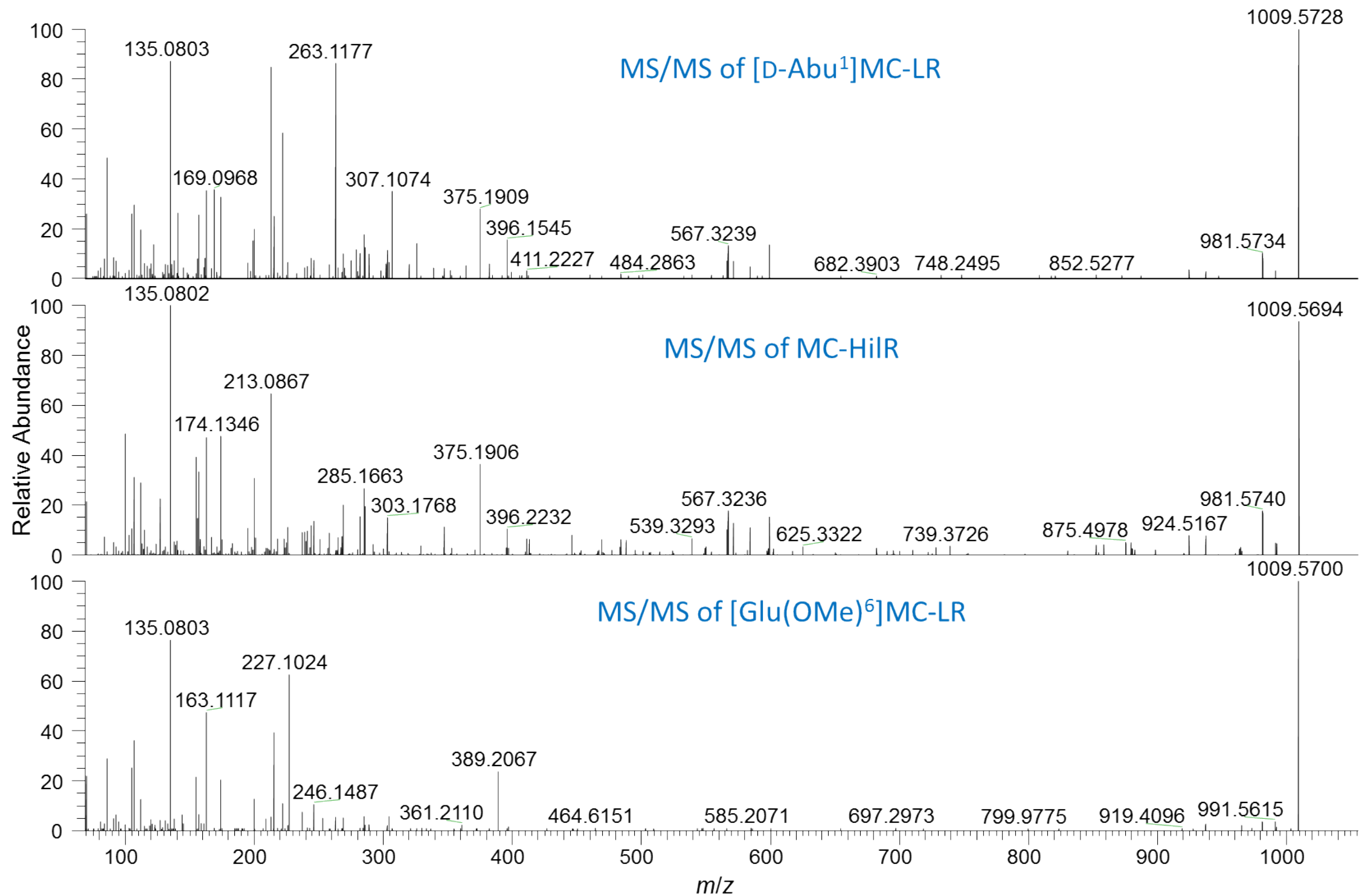

Figure S47. LC-MS PRM MS/MS spectra obtained in positive ionization mode for [Abu $\left.{ }^{1}\right]$ MC-LR (32), MC-HilR (29), and $\left[\mathrm{Glu}(\mathrm{OMe})^{6}\right] \mathrm{MC}-\mathrm{LR}$. 


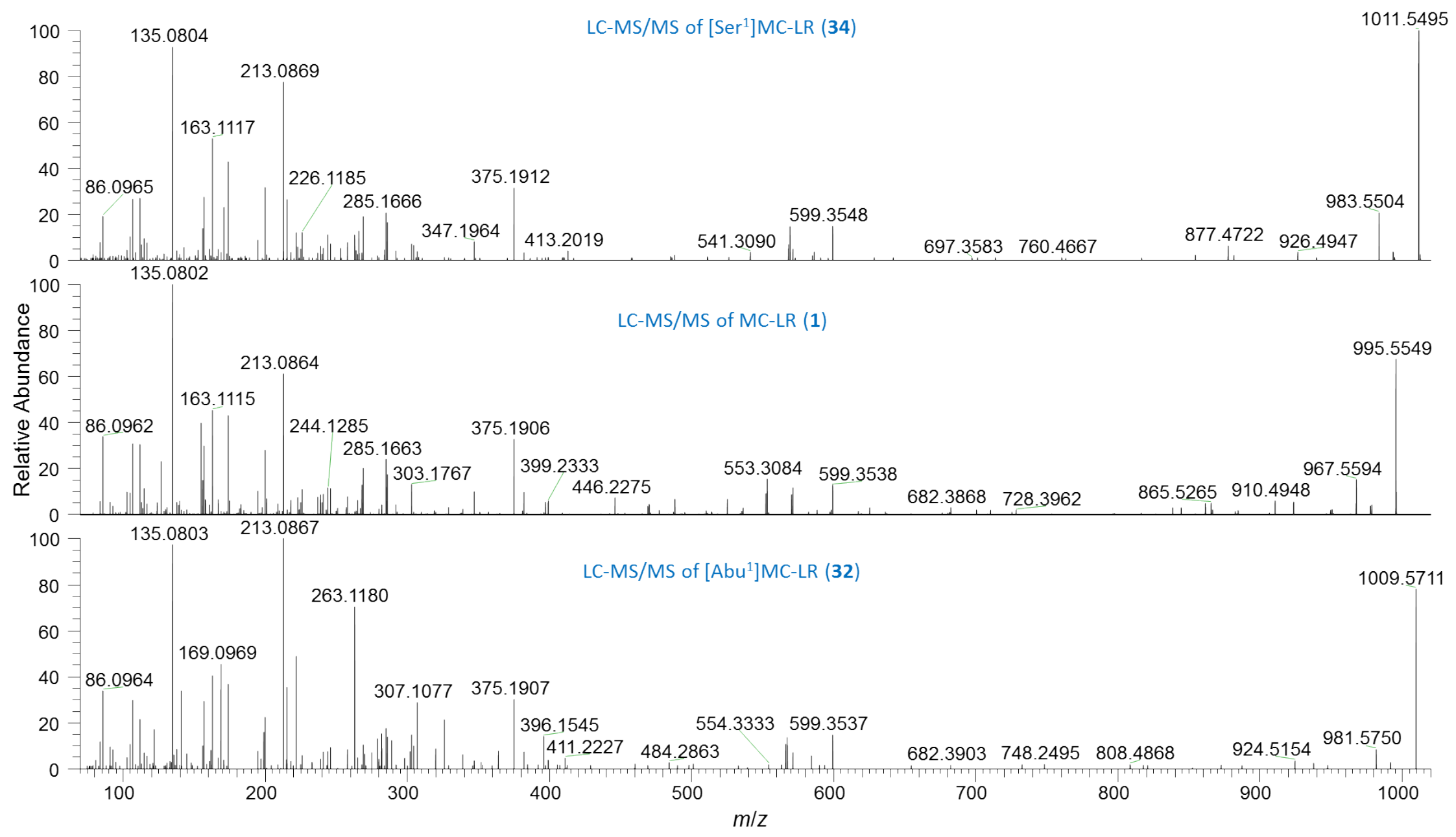

Figure S48. LC-MS PRM MS/MS spectra obtained in positive ionization mode for $[\mathrm{M}+\mathrm{H}]^{+}$of $\left[\operatorname{Ser}^{1}\right] \mathrm{MC}-\mathrm{LR}(\mathbf{3 4})$, MC-LR (1) and $\left[\mathrm{Abu}^{1}\right] \mathrm{MC}-\mathrm{LR}(\mathbf{3 2})$. 


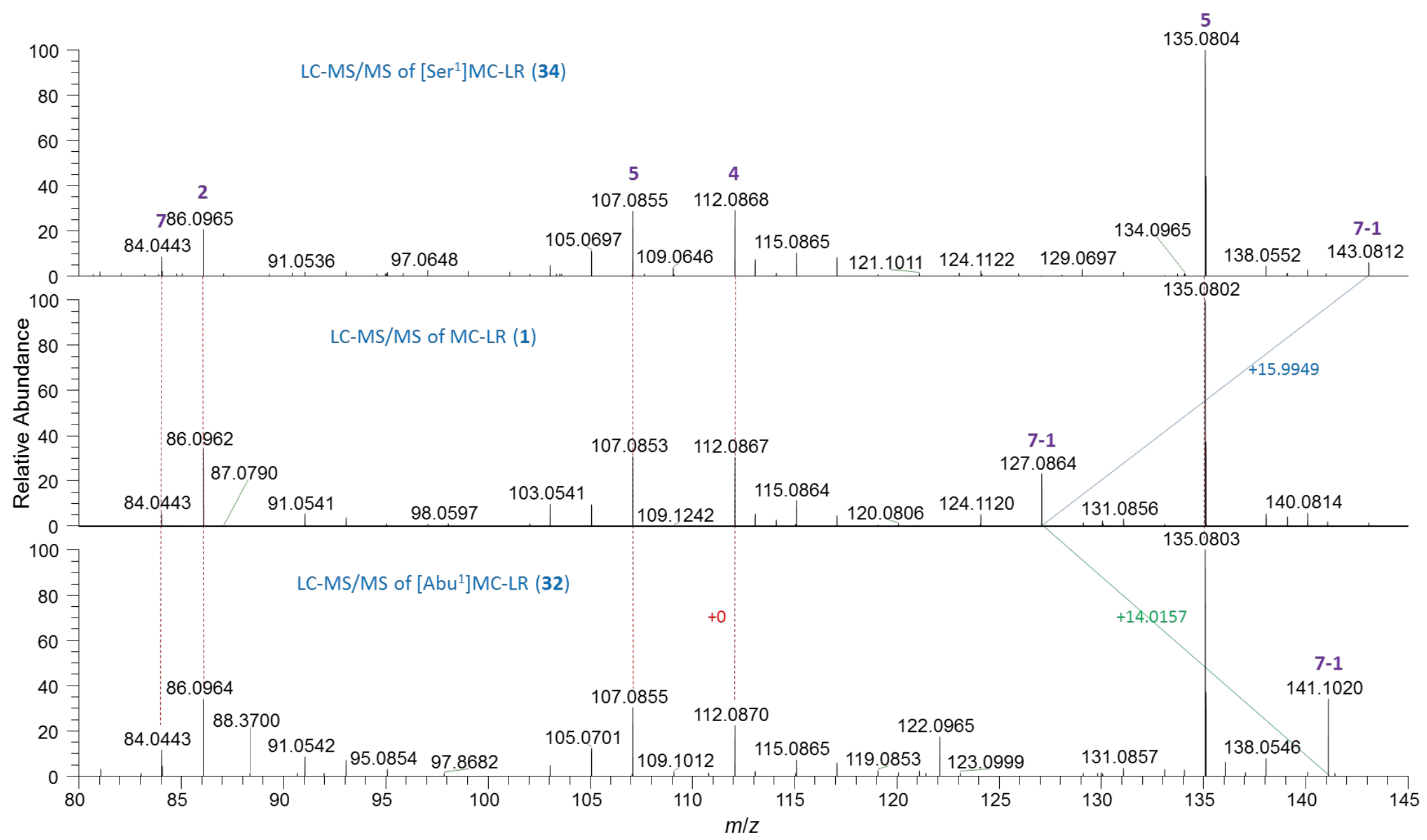

Figure S49. Expansion of LC-MS PRM MS/MS spectra obtained in positive ionization mode for [M+H] $]^{+}$of [Ser $\left.{ }^{1}\right] \mathrm{MC}-\mathrm{LR}(34)$, MCLR (1) and [ $\left.\mathrm{Abu}^{1}\right] \mathrm{MC}-\mathrm{LR}$ (32). Blue lines connect ions that increase relative to MC-LR by 15.9949 in 34, and green lines by 14.0157 in 32 (corresponding to $\mathrm{O}$, and $\mathrm{CH}_{2}$, respectively), while dashed red lines connect ions that remain unchanged. Bold purple numbers indicate amino acid residues associated with selected product ions in 1. Mass differences in product ions of $\mathbf{3 2}$ and $\mathbf{3 4}$ are seen only with changes in the amino acid at position-1. 


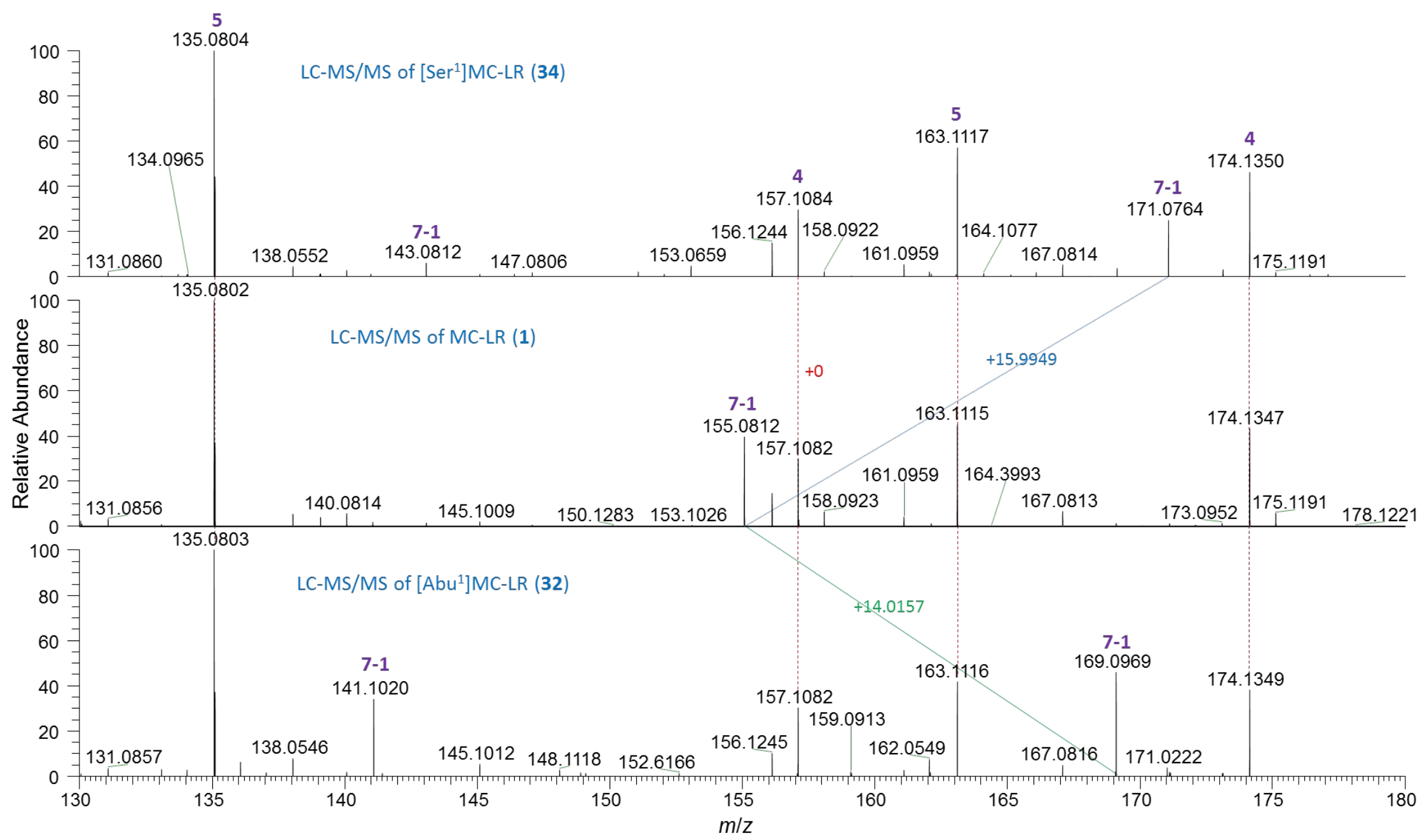

Figure S50. Expansion of LC-MS PRM MS/MS spectra obtained in positive ionization mode for [M+H] ${ }^{+}$of [Ser $\left.{ }^{1}\right] \mathrm{MC}-\mathrm{LR}(34)$, MCLR (1) and [ $\left.\mathrm{Abu}^{1}\right] \mathrm{MC}-\mathrm{LR}(\mathbf{3 2})$. Blue lines connect ions that increase relative to MC-LR by 15.9949 in 34, and green lines by 14.0157 in 32 (corresponding to $\mathrm{O}$, and $\mathrm{CH}_{2}$, respectively), while dashed red lines connect ions that remain unchanged. Bold purple numbers indicate amino acid residues associated with selected product ions in 1. Mass differences in product ions of $\mathbf{3 2}$ and $\mathbf{3 4}$ are seen only with changes in the amino acid at position-1. 


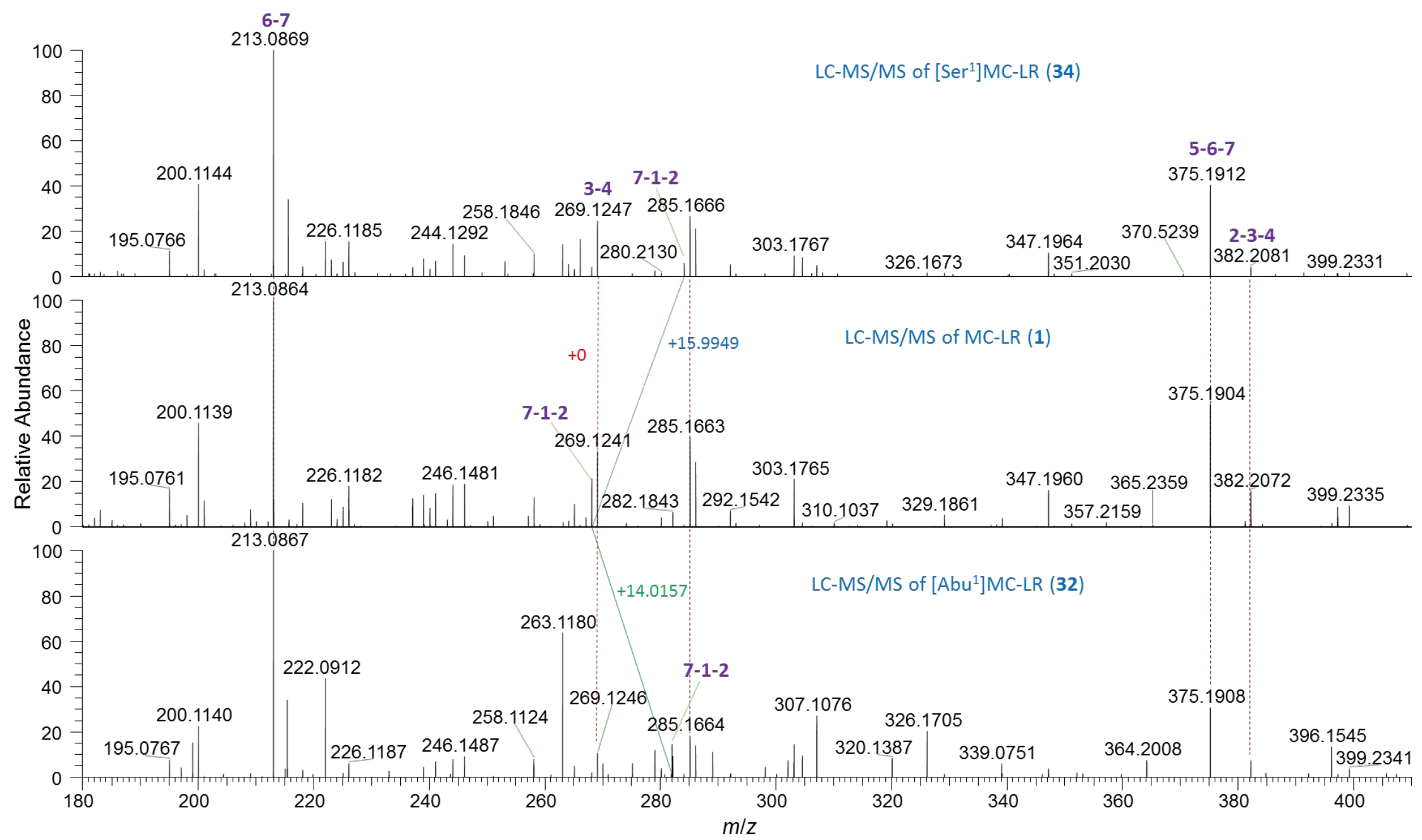

Figure S51. Expansion of LC-MS PRM MS/MS spectra obtained in positive ionization mode for $[\mathrm{M}+\mathrm{H}]^{+}$of $\left.^{[\mathrm{Ser}}{ }^{1}\right] \mathrm{MC}-\mathrm{LR}(\mathbf{3 4})$, MCLR (1) and [ $\left.\mathrm{Abu}^{1}\right] \mathrm{MC}-\mathrm{LR}(\mathbf{3 2})$. Blue lines connect ions that increase relative to MC-LR by 15.9949 in 34, and green lines by 14.0157 in 32 (corresponding to $\mathrm{O}$, and $\mathrm{CH}_{2}$, respectively), while dashed red lines connect ions that remain unchanged. Bold purple numbers indicate amino acid residues associated with selected product ions in $\mathbf{1}$. Mass differences in product ions of $\mathbf{3 2}$ and $\mathbf{3 4}$ are seen only with changes in the amino acid at position-1. 


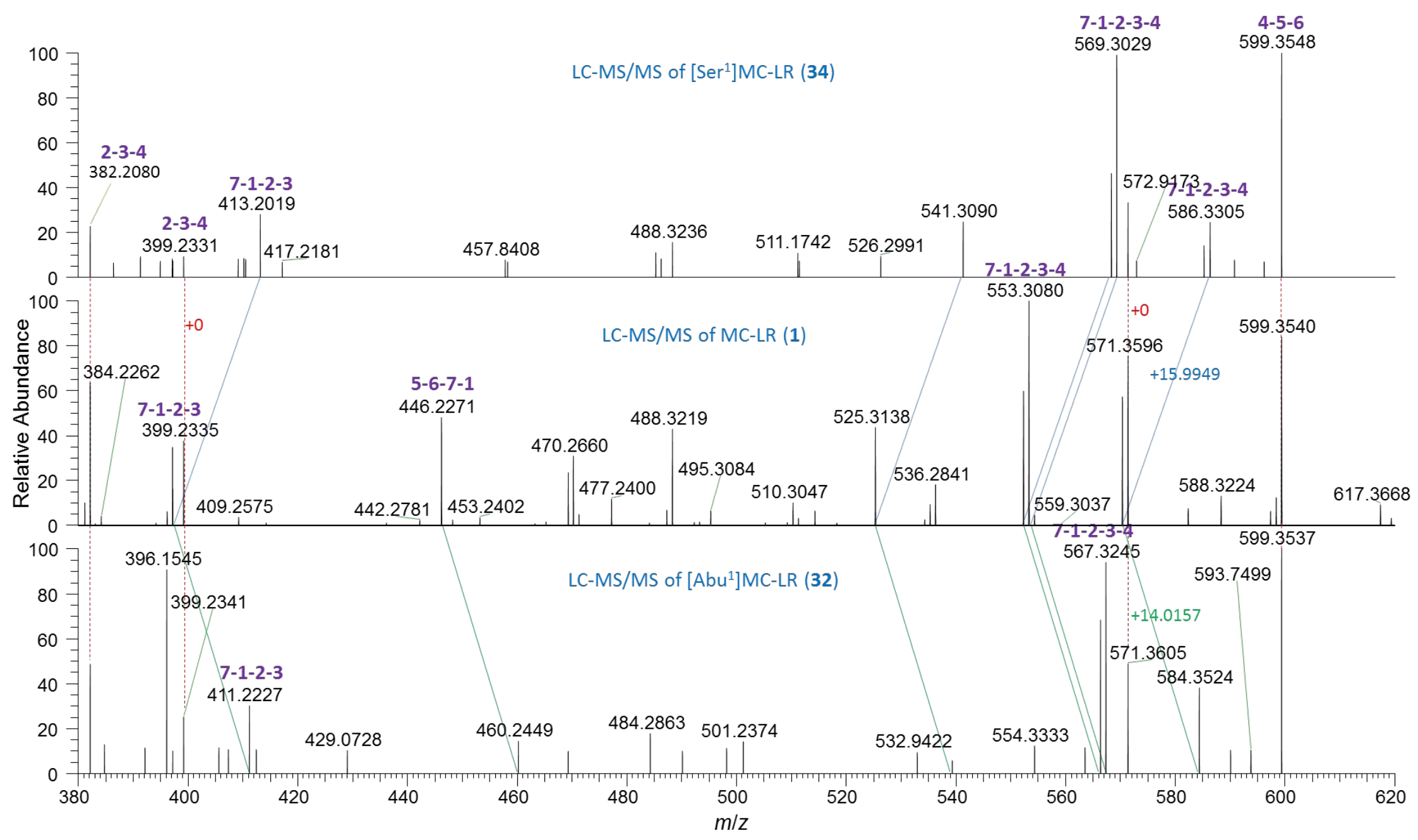

Figure S52. Expansion of LC-MS PRM MS/MS spectra obtained in positive ionization mode for [M+H] $]^{+}$of [Ser $\left.{ }^{1}\right] \mathrm{MC}-\mathrm{LR}(\mathbf{3 4})$, MCLR (1) and [ $\left.\mathrm{Abu}^{1}\right] \mathrm{MC}-\mathrm{LR}$ (32). Blue lines connect ions that increase relative to MC-LR by 15.9949 in 34, and green lines by 14.0157 in 32 (corresponding to $\mathrm{O}$, and $\mathrm{CH}_{2}$, respectively), while dashed red lines connect ions that remain unchanged. Bold purple numbers indicate amino acid residues associated with selected product ions in 1. Mass differences in product ions of $\mathbf{3 2}$ and $\mathbf{3 4}$ are seen only with changes in the amino acid at position-1. 


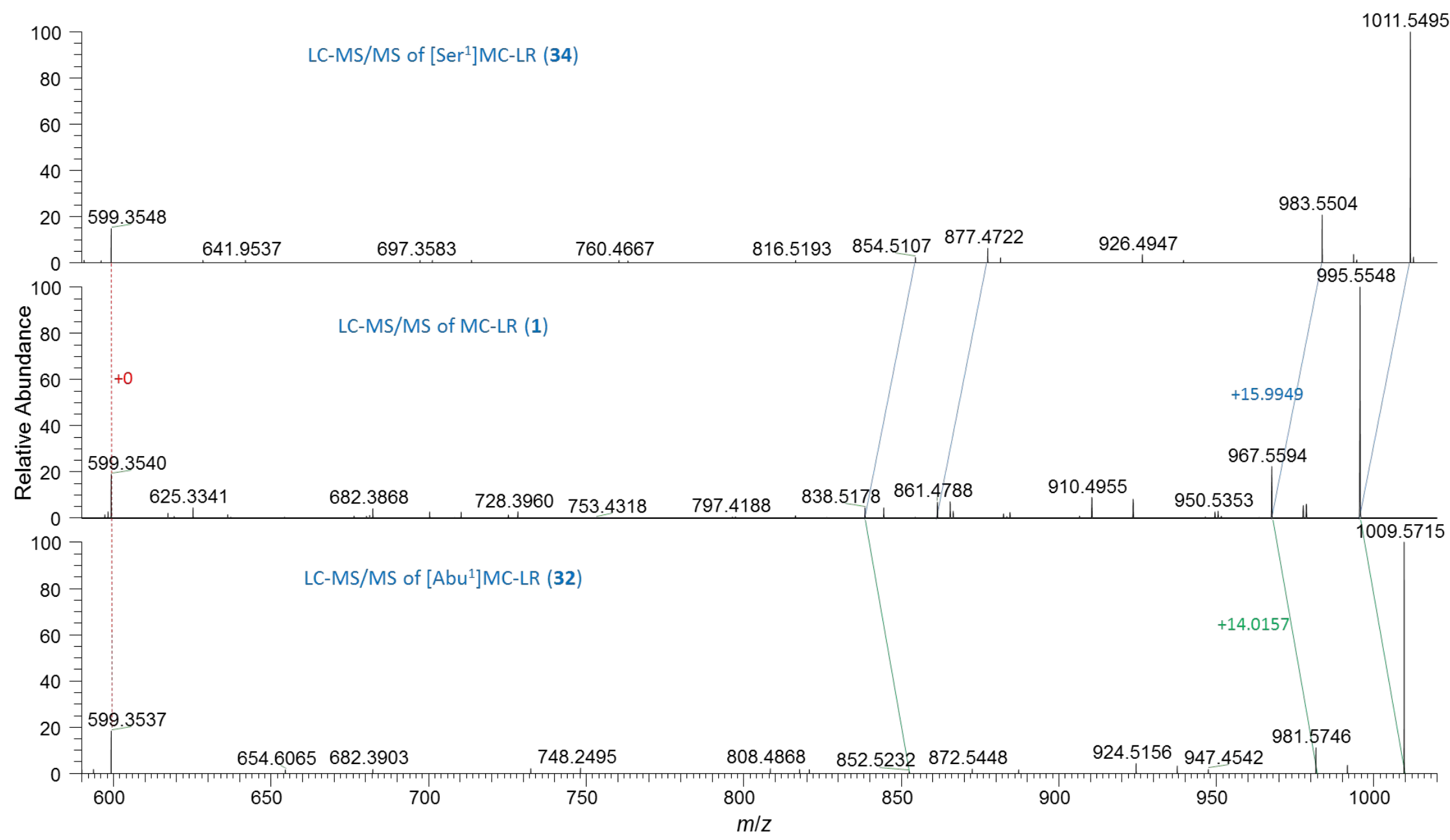

Figure S53. Expansion of LC-MS PRM MS/MS spectra obtained in positive ionization mode for $[\mathrm{M}+\mathrm{H}]^{+}$of $\left[\mathrm{Ser}{ }^{1}\right] \mathrm{MC}-\mathrm{LR}(\mathbf{3 4})$, MCLR (1) and [ $\left.\mathrm{Abu}^{1}\right] \mathrm{MC}-\mathrm{LR}(\mathbf{3 2})$. Blue lines connect ions that increase relative to MC-LR by 15.9949 in 34, and green lines by 14.0157 in 32 (corresponding to $\mathrm{O}$, and $\mathrm{CH}_{2}$, respectively), while dashed red lines connect ions that remain unchanged. Bold purple numbers indicate amino acid residues associated with selected product ions in $\mathbf{1}$. Mass differences in product ions of $\mathbf{3 2}$ and $\mathbf{3 4}$ are seen only with changes in the amino acid at position-1. 


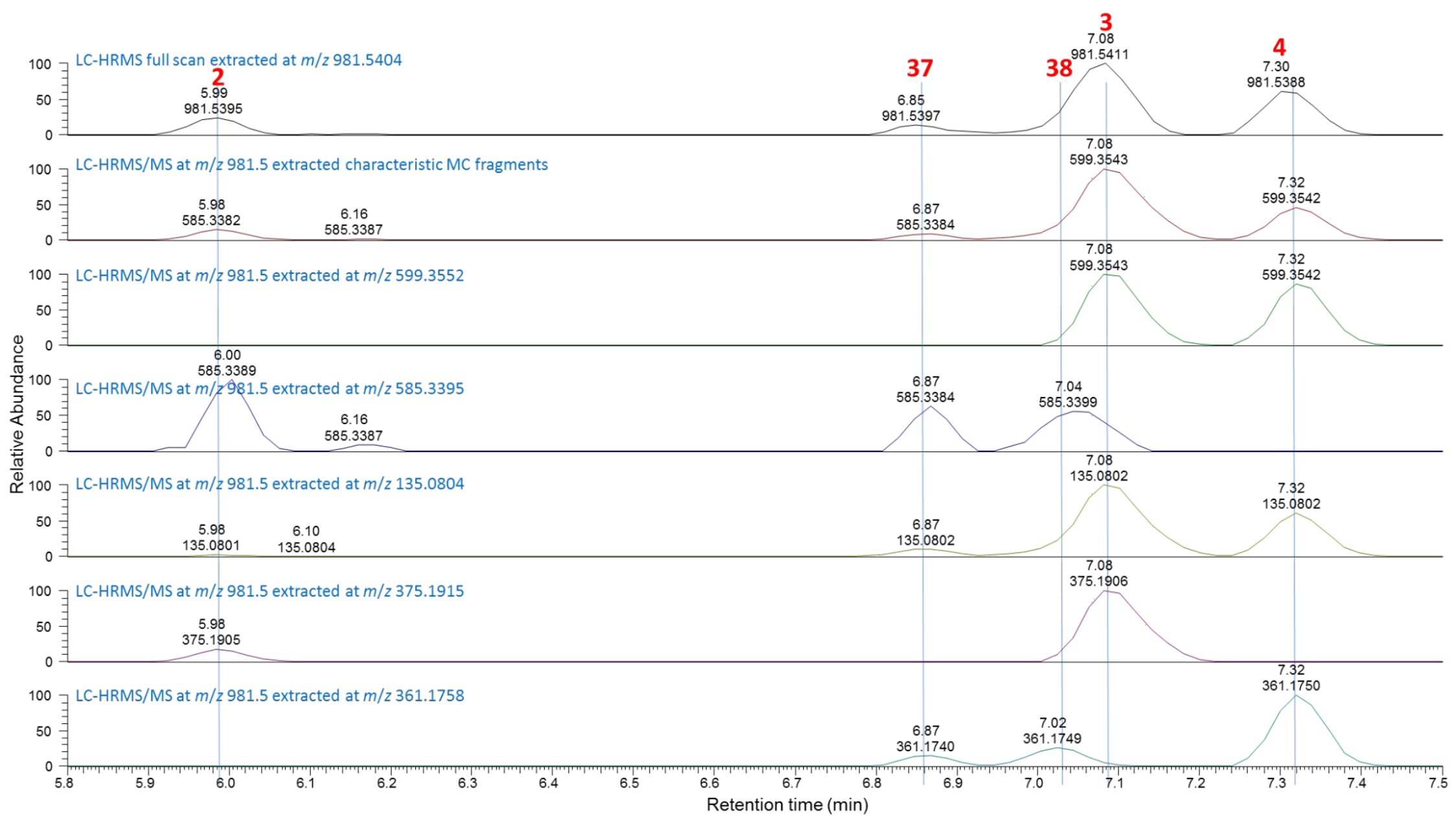

Figure S54. Top, full-scan positive mode LC-HRMS chromatograms extracted at the exact $[\mathrm{M}+\mathrm{H}]^{+} m / z$ for desmethylMC-LR, and; underneath are LC-MS PRM chromatograms at $\mathrm{m} / \mathrm{z} 981.5$ extracted for selected $\mathrm{m} / \mathrm{z}$ of microcystin product ions that are characteristic for MC-LR or desmethylMC-LR. The results reveal the presence of the know desmethylMC-LR congeners [DMAdda $\left.{ }^{5}\right]$ MC-LR (2), $\left[\mathrm{Asp}^{3}\right] \mathrm{MC}-\mathrm{LR}(\mathbf{3})$, and $\left[\mathrm{Dha}^{7}\right] \mathrm{MC}-\mathrm{LR}(\mathbf{4})$, as well as two congeners (37 and 38) in which $\mathrm{CH}_{2}$ is missing from MC-LR somewhere in the Adda side-chain somewhere between $\mathrm{C}-2$ and $\mathrm{C}-8$. 


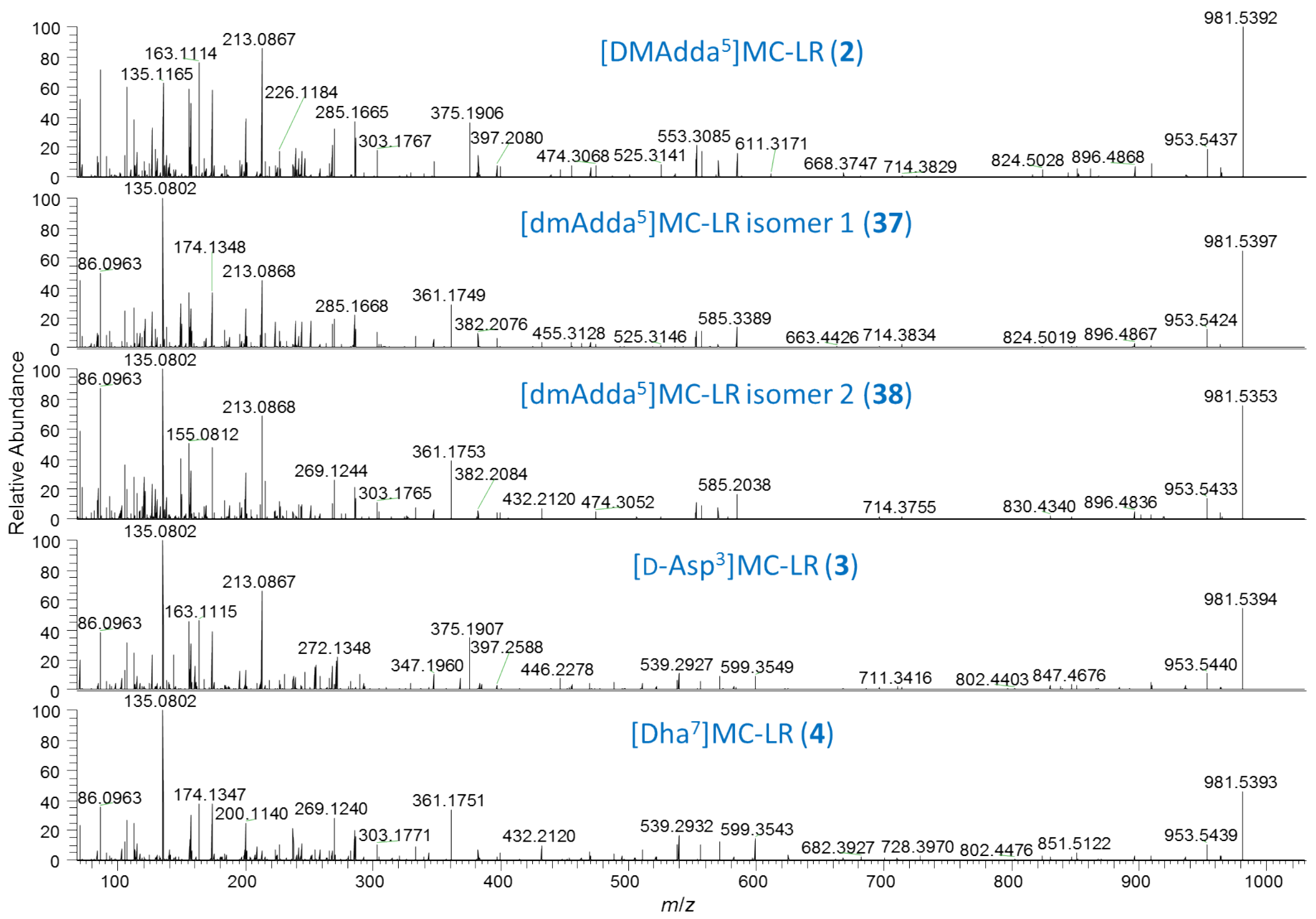

Figure S55. LC-MS PRM MS/MS spectra obtained in positive ionization mode for $[\mathrm{M}+\mathrm{H}]^{+}$of $\left[\mathrm{DMAdda}{ }^{5}\right] \mathrm{MC}-\mathrm{LR}(2)$, [dmAdda $\left.{ }^{5}\right] \mathrm{MC}-\mathrm{LR}$ isomer 1 (37), [dmAdda $\left.{ }^{5}\right] \mathrm{MC}-\mathrm{LR}$ isomer 2 (38), [Asp $\left.{ }^{3}\right] \mathrm{MC}-\mathrm{LR}$ (3), and [Dha $\left.{ }^{7}\right] \mathrm{MC}-\mathrm{LR}$ (4). 


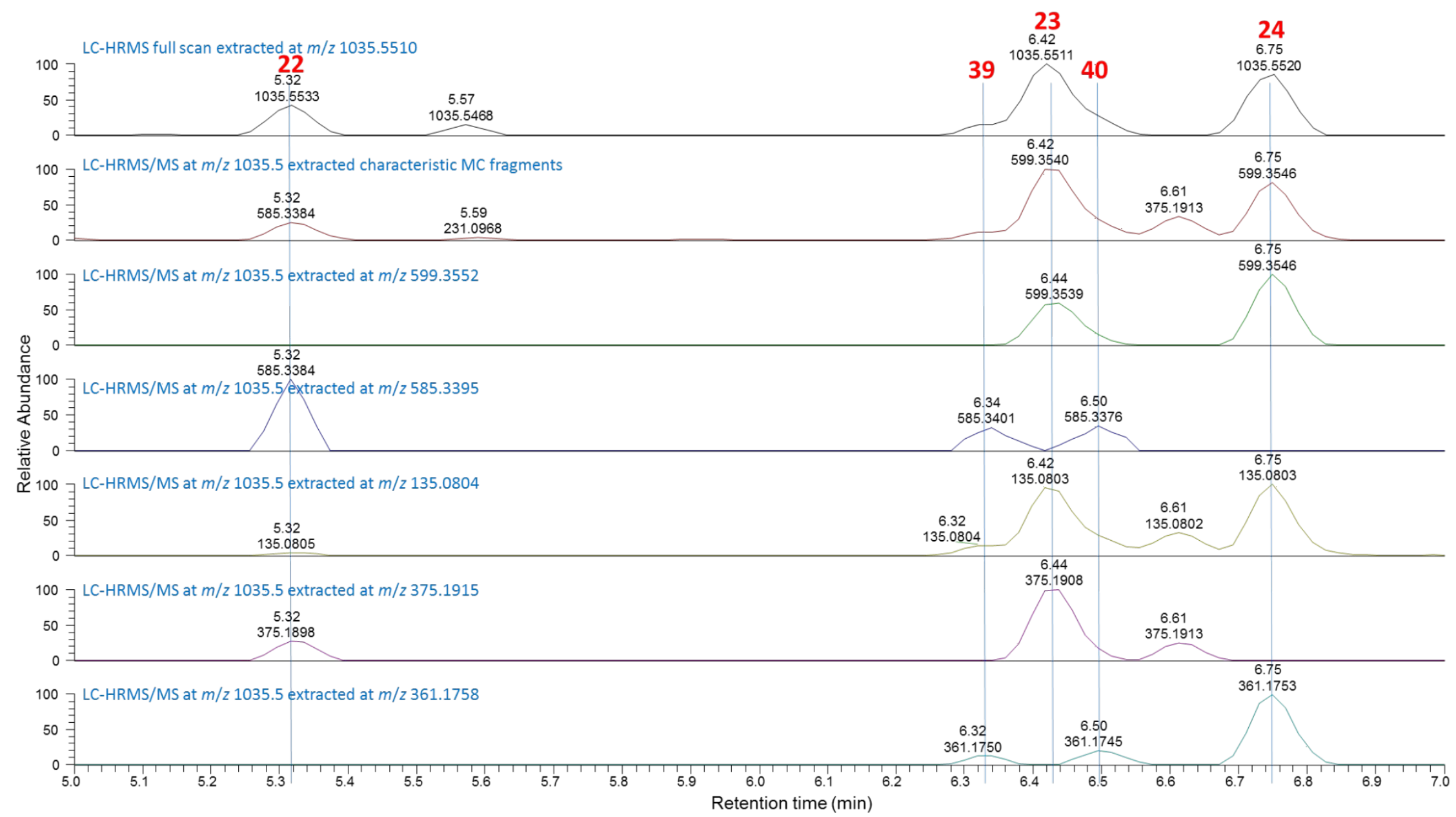

Figure S56. Top, full-scan positive mode LC-HRMS chromatograms extracted at the exact $[\mathrm{M}+\mathrm{H}]^{+} m / z$ for desmethylMC-(H4)YR, and; underneath are LC-MS PRM chromatograms at $\mathrm{m} / \mathrm{z} 1035.5$ extracted for selected $\mathrm{m} / \mathrm{z}$ of microcystin product ions that are characteristic for MC-(H4)YR or desmethylMC-(H4)YR. The results reveal the presence of the know desmethylMC-(H4)YR congeners [DMAdda ${ }^{5}$ MC-(H4)YR (22), [Asp $\left.{ }^{3}\right] \mathrm{MC}-(\mathrm{H} 4) \mathrm{YR}$ (23), and [Dha $\left.{ }^{7}\right] \mathrm{MC}-(\mathrm{H} 4) \mathrm{YR}$ (24), as well as two congeners (39 and 40) in which $\mathrm{CH}_{2}$ is missing from $\mathrm{MC}-(\mathrm{H} 4) \mathrm{YR}$ somewhere in the Adda side-chain somewhere between $\mathrm{C}-2$ and $\mathrm{C}-8$. 


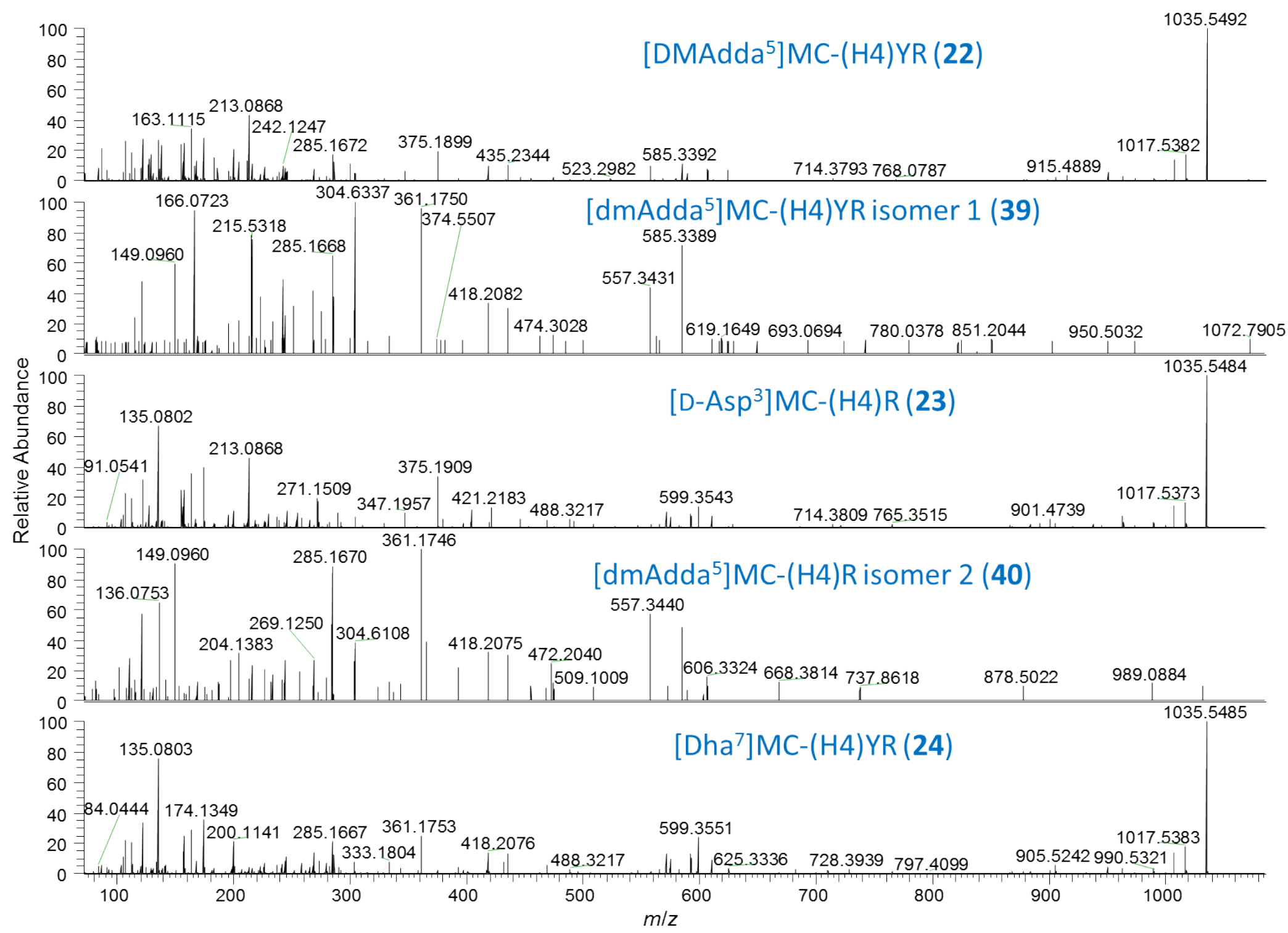

Figure S57. LC-MS PRM MS/MS spectra obtained in positive ionization mode for $[\mathrm{M}+\mathrm{H}]^{+}$of $[\mathrm{DMAdda}] \mathrm{MC}-(\mathrm{H} 4) \mathrm{YR}(\mathbf{2 2})$, [dmAdda $\left.{ }^{5}\right] \mathrm{MC}-(\mathrm{H} 4) Y R$ isomer 1 (39), [Asp $\left.{ }^{3}\right] \mathrm{MC}-(\mathrm{H} 4) \mathrm{YR}(\mathbf{2 3}),\left[\mathrm{dmAdda}^{5}\right] \mathrm{MC}-(\mathrm{H} 4) \mathrm{YR}$ isomer 2 (40), and [Dha7 ${ }^{7} \mathrm{MC}-(\mathrm{H} 4) \mathrm{YR}(\mathbf{2 4})$. 

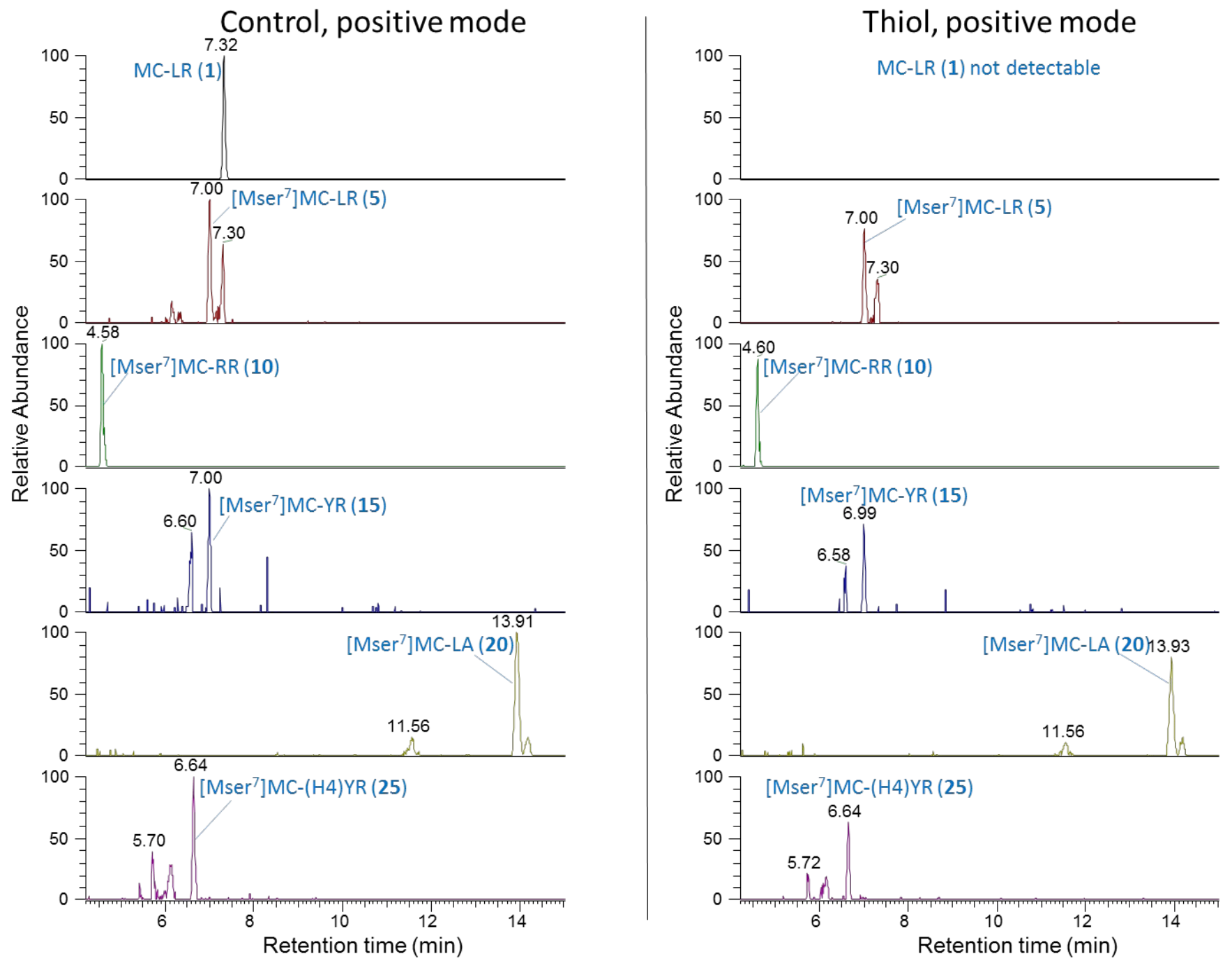

Figure S58. Full-scan positive mode LC-HRMS chromatograms extracted at the exact $[\mathrm{M}+\mathrm{H}]^{+} m / z$ for MC-LR (1), [Mser $\left.{ }^{7}\right] \mathrm{MC}-\mathrm{LR}$ (5), [Mser $\left.{ }^{7}\right] \mathrm{MC}-\mathrm{RR}$ (10), [Mser $\left.{ }^{7}\right] \mathrm{MC}-\mathrm{YR}$ (15), [Mser $\left.{ }^{7}\right] \mathrm{MC}-\mathrm{LA}$ (20), and [Mser $\left.{ }^{7}\right] \mathrm{MC}-(\mathrm{H} 4) \mathrm{YR}$ (25), presented on the same vertical scale. Right, after derivatization with mercaptoethanol, and; left, in a buffered control sample. The Mhda ${ }^{7}$-containing MC-LR (1) was almost completely converted, whereas Mser ${ }^{7}$-containing MCs were only slightly reduced in intensity by treatment with the thiol. 


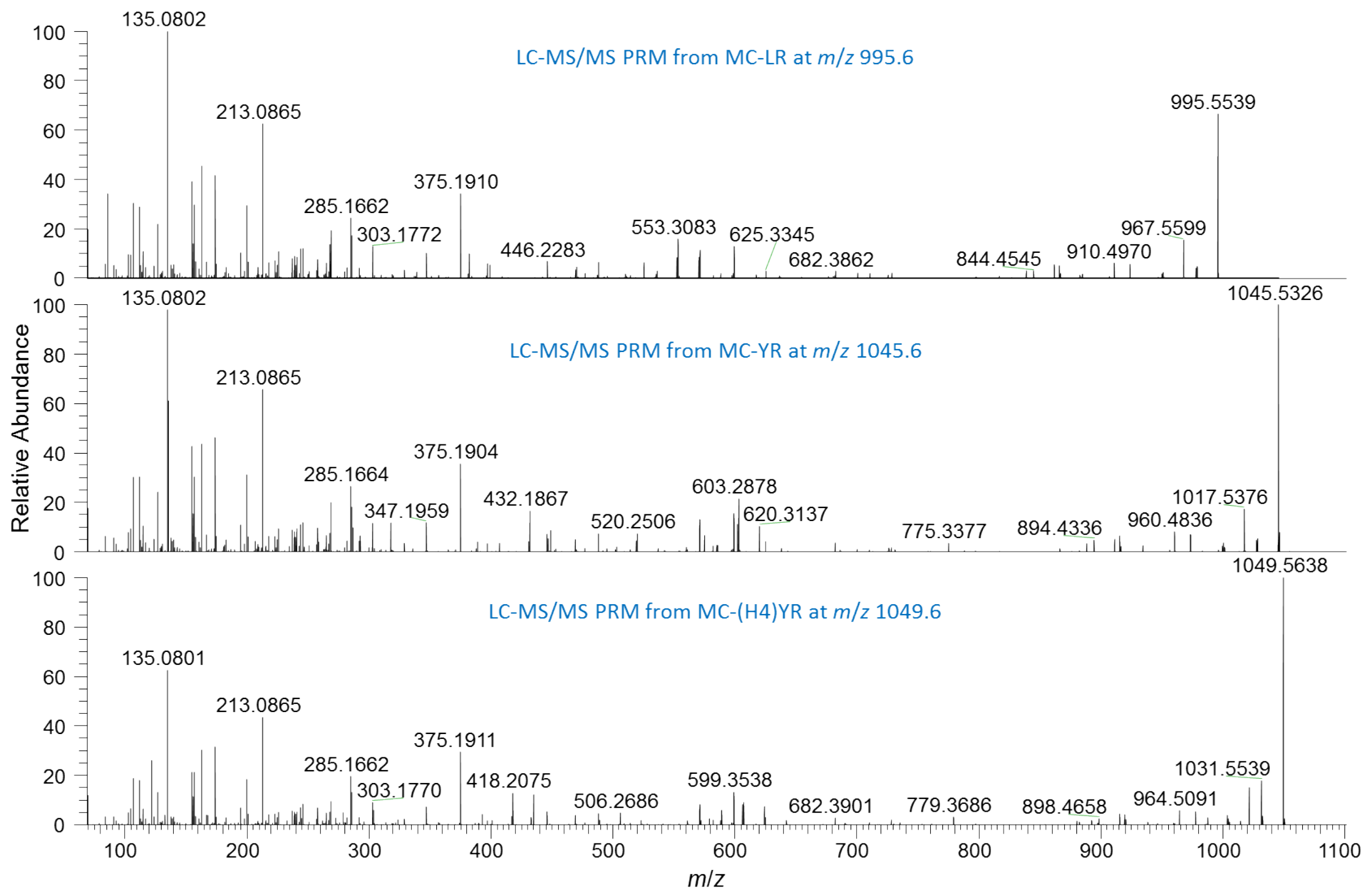

Figure S59. LC-MS PRM MS/MS spectra obtained in positive ionization mode for $[\mathrm{M}+\mathrm{H}]^{+}$of MC-LR (1), MC-YR (11), and MC(H4)YR (21). 

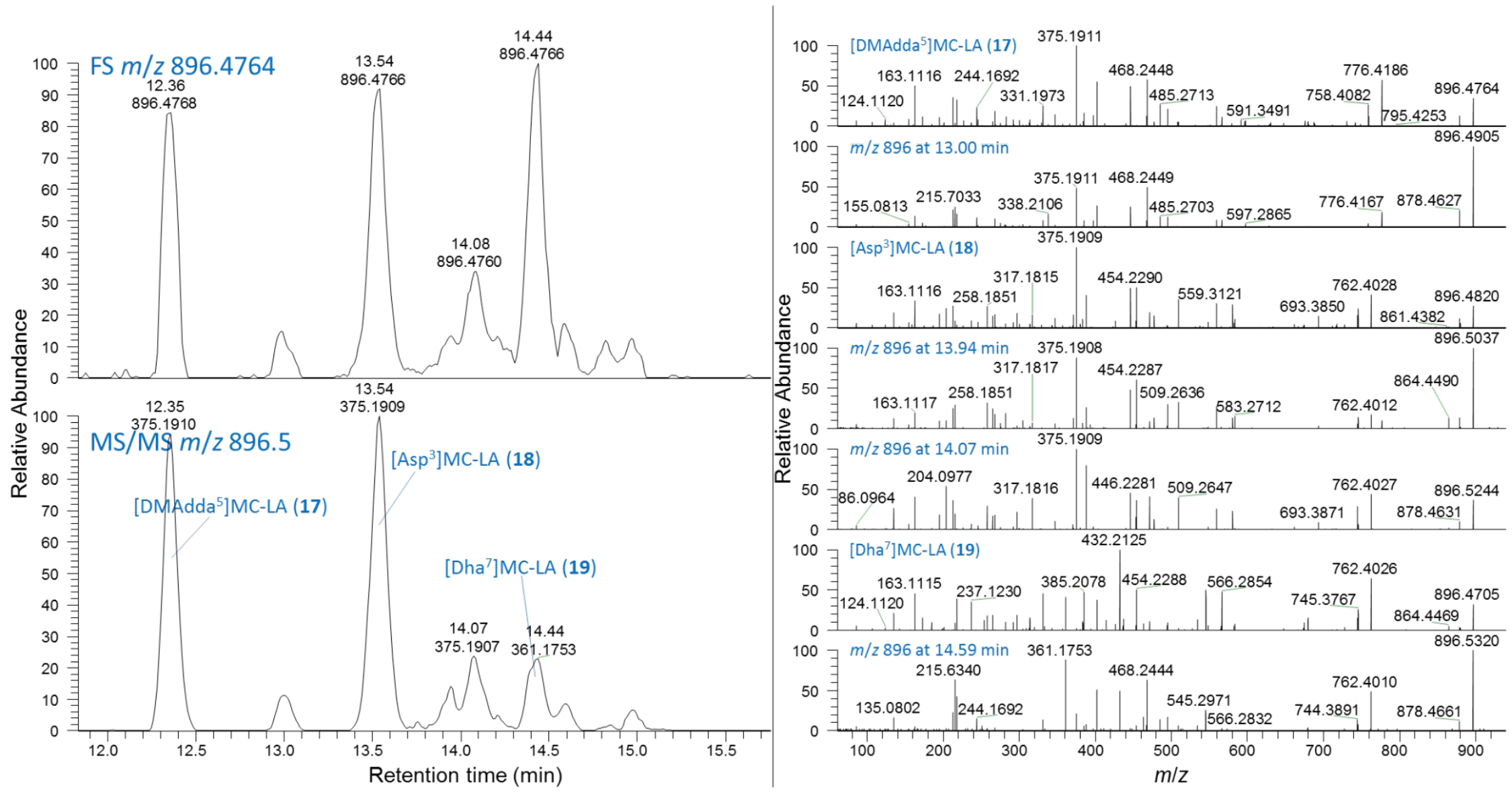

Figure S60. Top left, full-scan positive mode LC-HRMS chromatograms extracted at the exact $[\mathrm{M}+\mathrm{H}]^{+} \mathrm{m} / z$ for desmethylMC-LA, and; bottom left, LC-MS PRM chromatogram at $\mathrm{m} / \mathrm{z} 896.5$ extracted for $\mathrm{m} / \mathrm{z}$ of the characteristic microcystin product ions. Peaks identified as $\left[\mathrm{DMAdda}^{5}\right] \mathrm{MC}-\mathrm{LA}(\mathbf{1 7}),\left[\mathrm{Asp}^{3}\right] \mathrm{MC}-\mathrm{LA}(\mathbf{1 8})$, and $\left[\mathrm{Dha}^{7}\right] \mathrm{MC}-\mathrm{LA}(\mathbf{1 9})$ are indicated, and corresponding MS/MS spectra are shown on the right. 


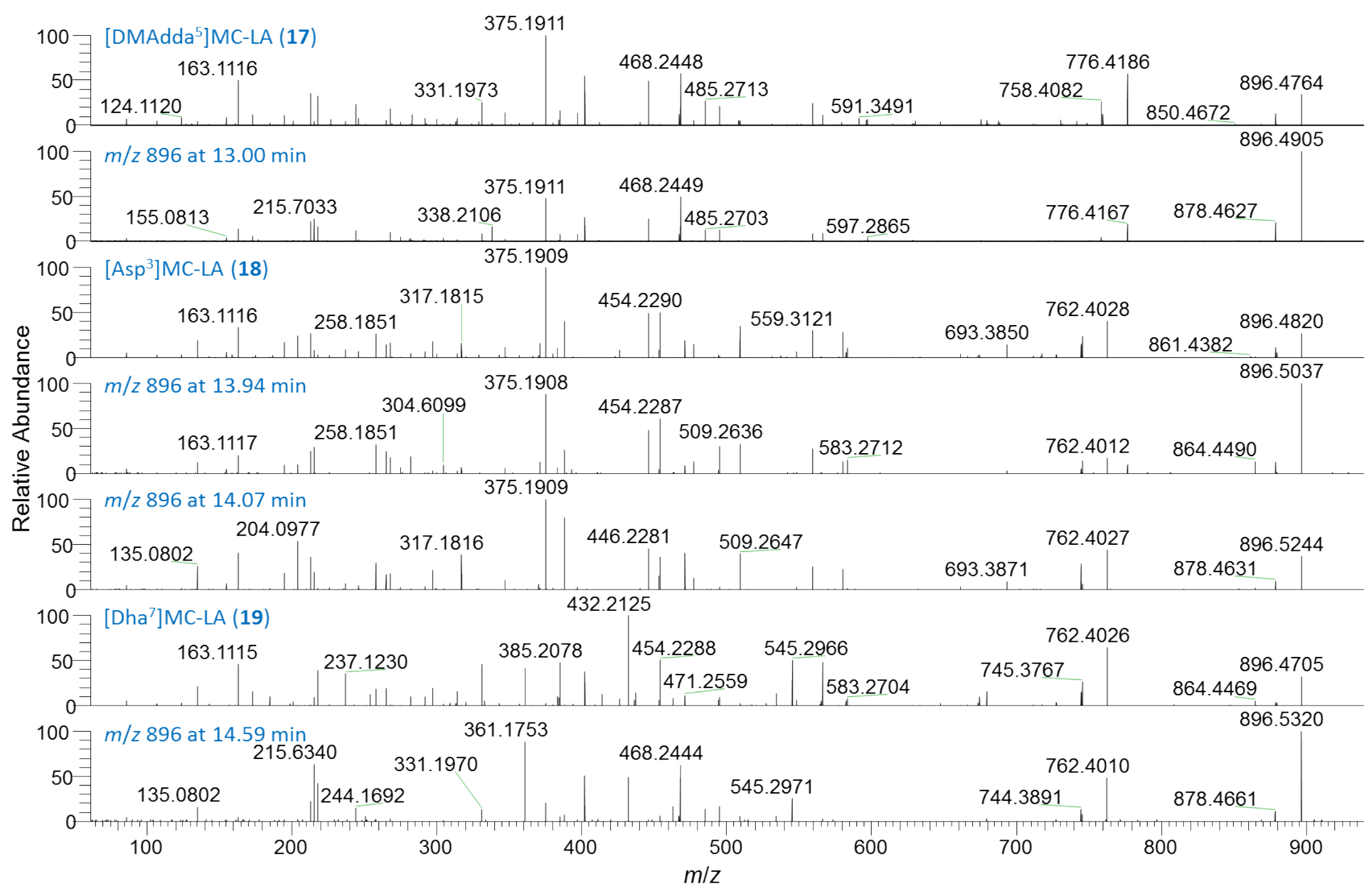

Figure S61. HRMS/MS spectra of desmethylMC-LA congeners $[\mathrm{M}+\mathrm{H}]^{+}$of [DMAdda $\left.{ }^{5}\right] \mathrm{MC}-\mathrm{LA}(\mathbf{1 7})$, [Asp $\left.{ }^{3}\right] \mathrm{MC}-\mathrm{LA}(\mathbf{1 8})$, and $\left[\mathrm{Dha}^{7}\right] \mathrm{MC}-\mathrm{LA}(\mathbf{1 9})$ and isobaric MC congeners detected in LC-HRMS/MS chromatograms in Figure S60. 

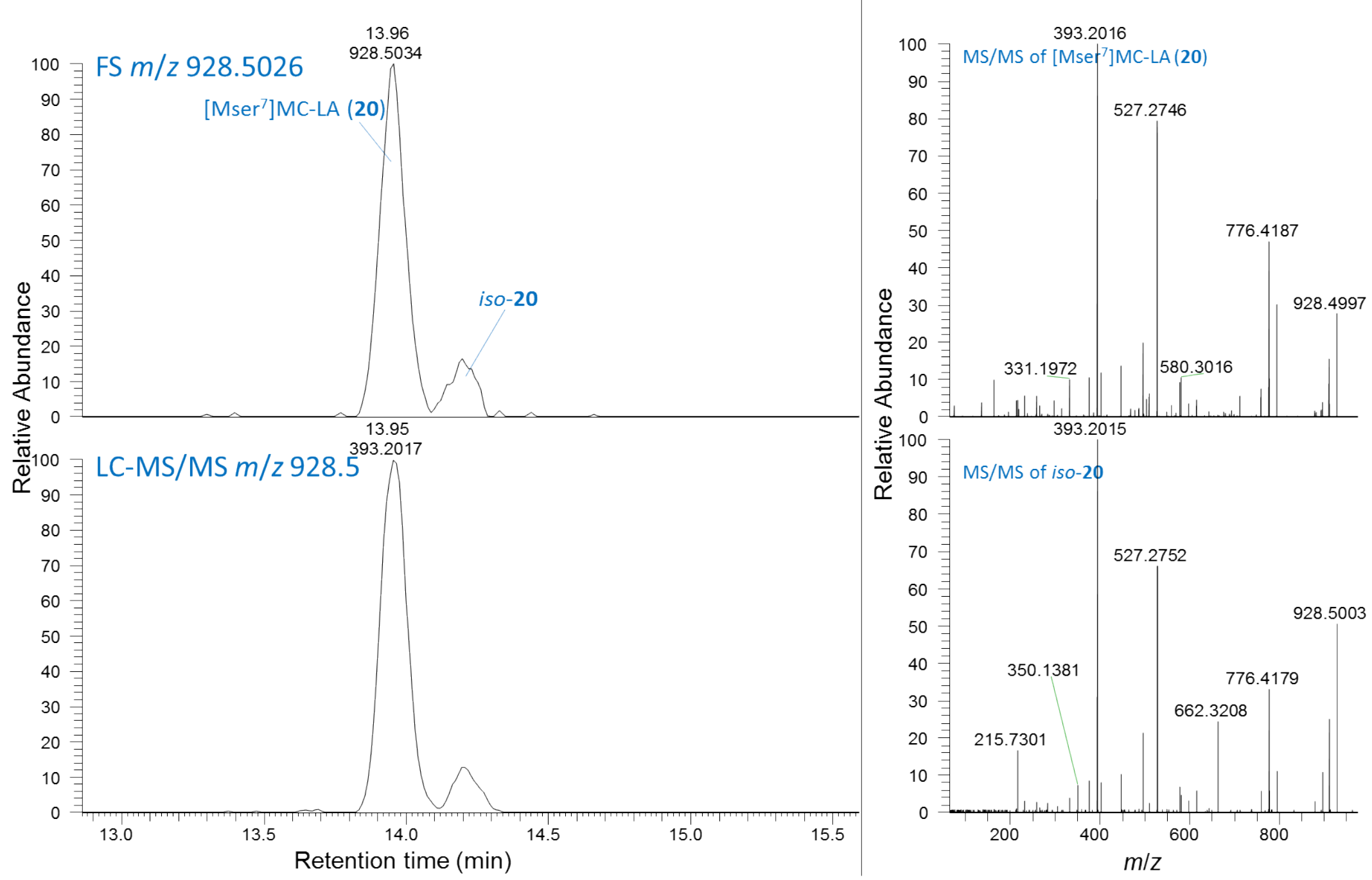

Figure S62. Top left, full-scan positive mode LC-HRMS chromatograms extracted at the exact $[\mathrm{M}+\mathrm{H}]^{+} m / z$ for $\left[\mathrm{Mser}{ }^{7}\right] \mathrm{MC}-\mathrm{LA}(\mathbf{2 0})$, and; bottom left, LC-MS PRM chromatogram at $\mathrm{m} / \mathrm{z} 928.5$ extracted for $\mathrm{m} / \mathrm{z}$ of the characteristic microcystin product ions. HRMS/MS spectra from the corresponding peaks are shown on the right. 


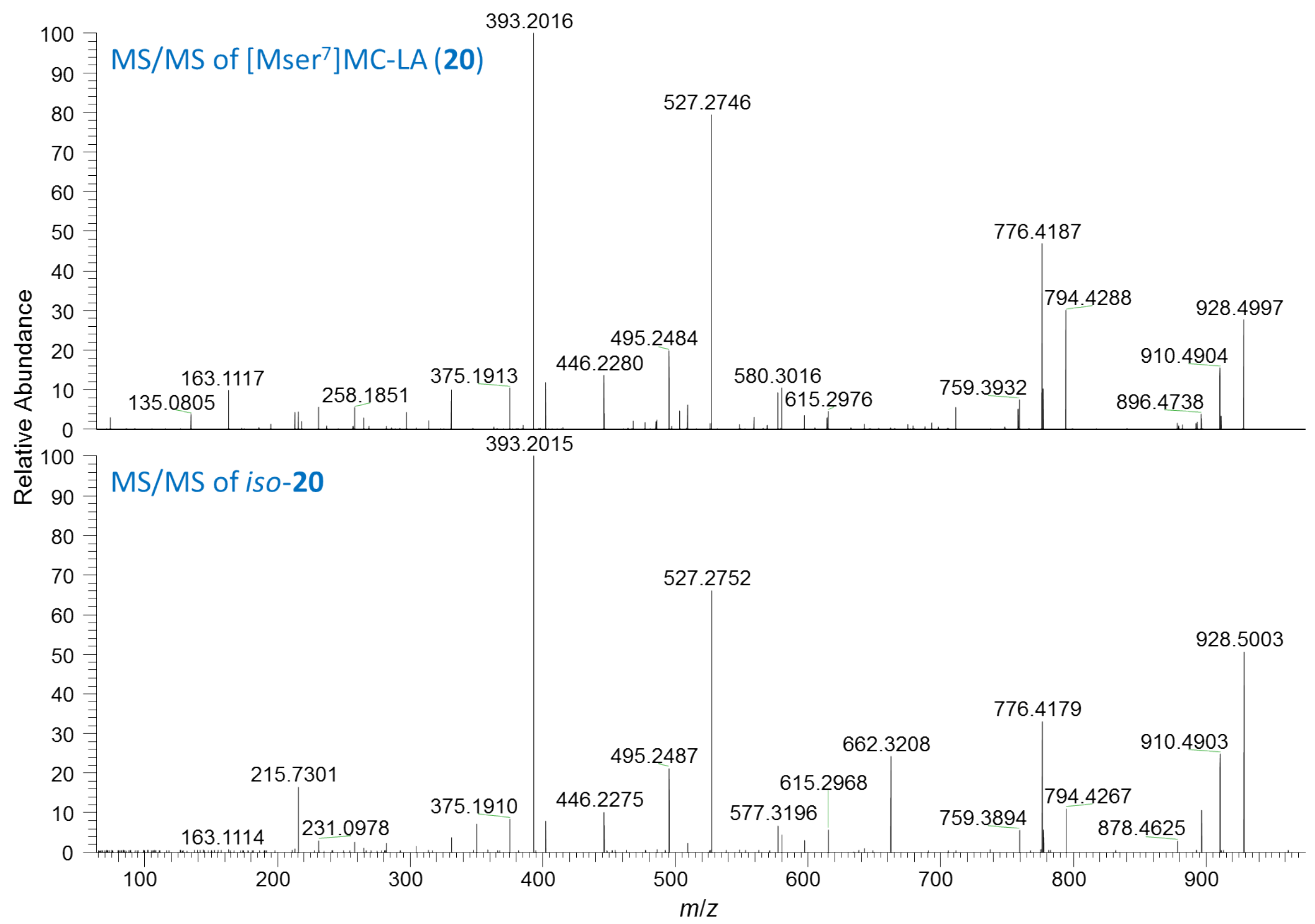

Figure S63. HRMS/MS spectra of $[\mathrm{M}+\mathrm{H}]^{+}$of $\left[\mathrm{Mser}^{7}\right] \mathrm{MC}-\mathrm{LA}(\mathbf{2 0})$ and an isomeric congener detected in LC-HRMS/MS chromatograms in Figure S62. 


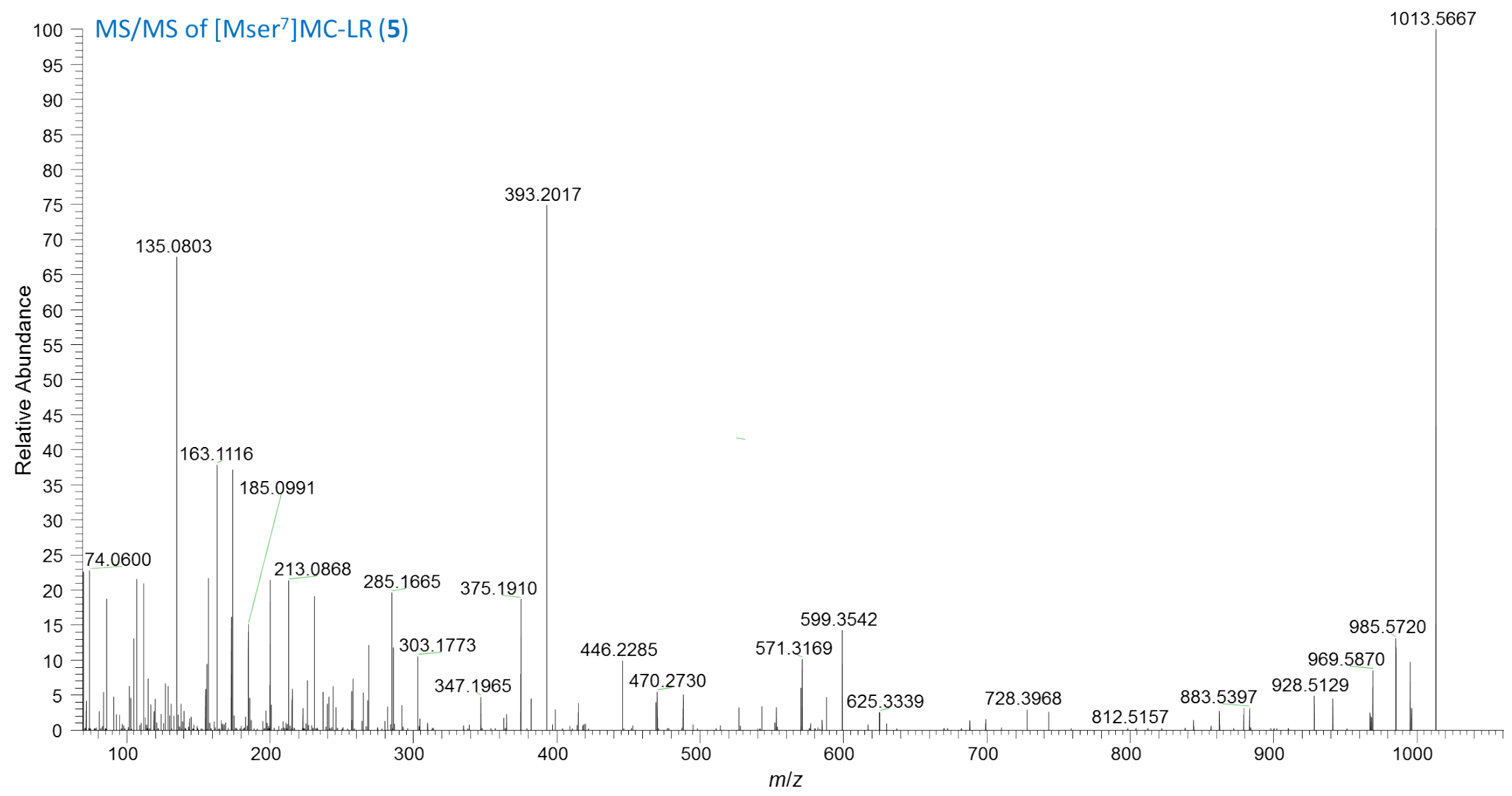

Figure S64. HRMS/MS spectrum of $[\mathrm{M}+\mathrm{H}]^{+}$of $\left[\mathrm{Mser}^{7}\right] \mathrm{MC}-\mathrm{LR}(5)$. 

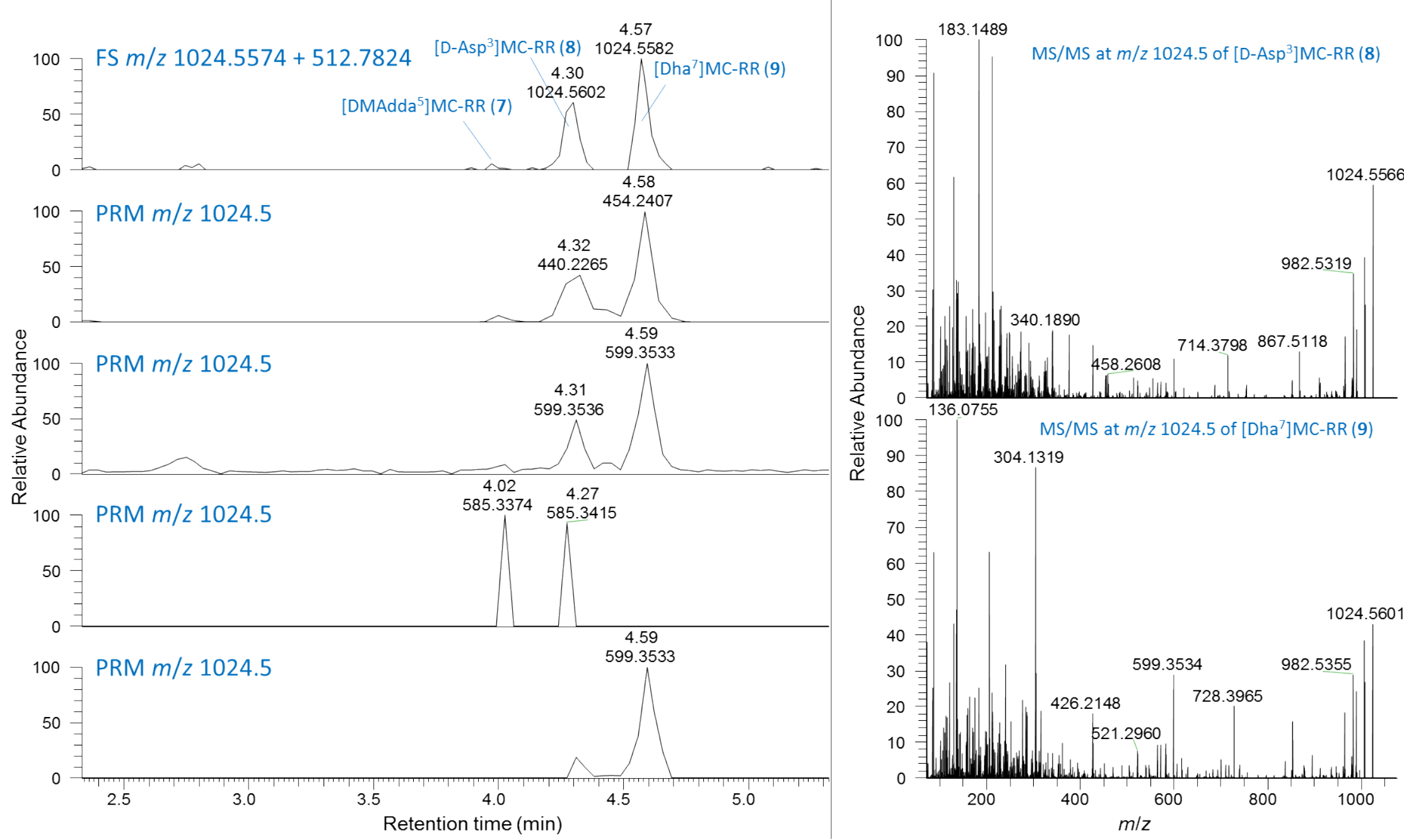

Figure S65. Top left, full-scan positive mode LC-HRMS chromatograms extracted at the exact $[\mathrm{M}+\mathrm{H}]^{+} m / z$ for desmethylMC-RR (79), and; below left, LC-MS PRM chromatograms at $\mathrm{m} / \mathrm{z} 1024.5$ extracted for $\mathrm{m} / \mathrm{z}$ of a range of characteristic microcystin product ions. HRMS/MS spectra from the corresponding peaks of [Asp $\left.{ }^{3}\right] \mathrm{MC}-\mathrm{RR}(\mathbf{8})$ and [Dha $\left.{ }^{7}\right] \mathrm{MC}-\mathrm{RR}(\mathbf{9})$ are shown on the right. 


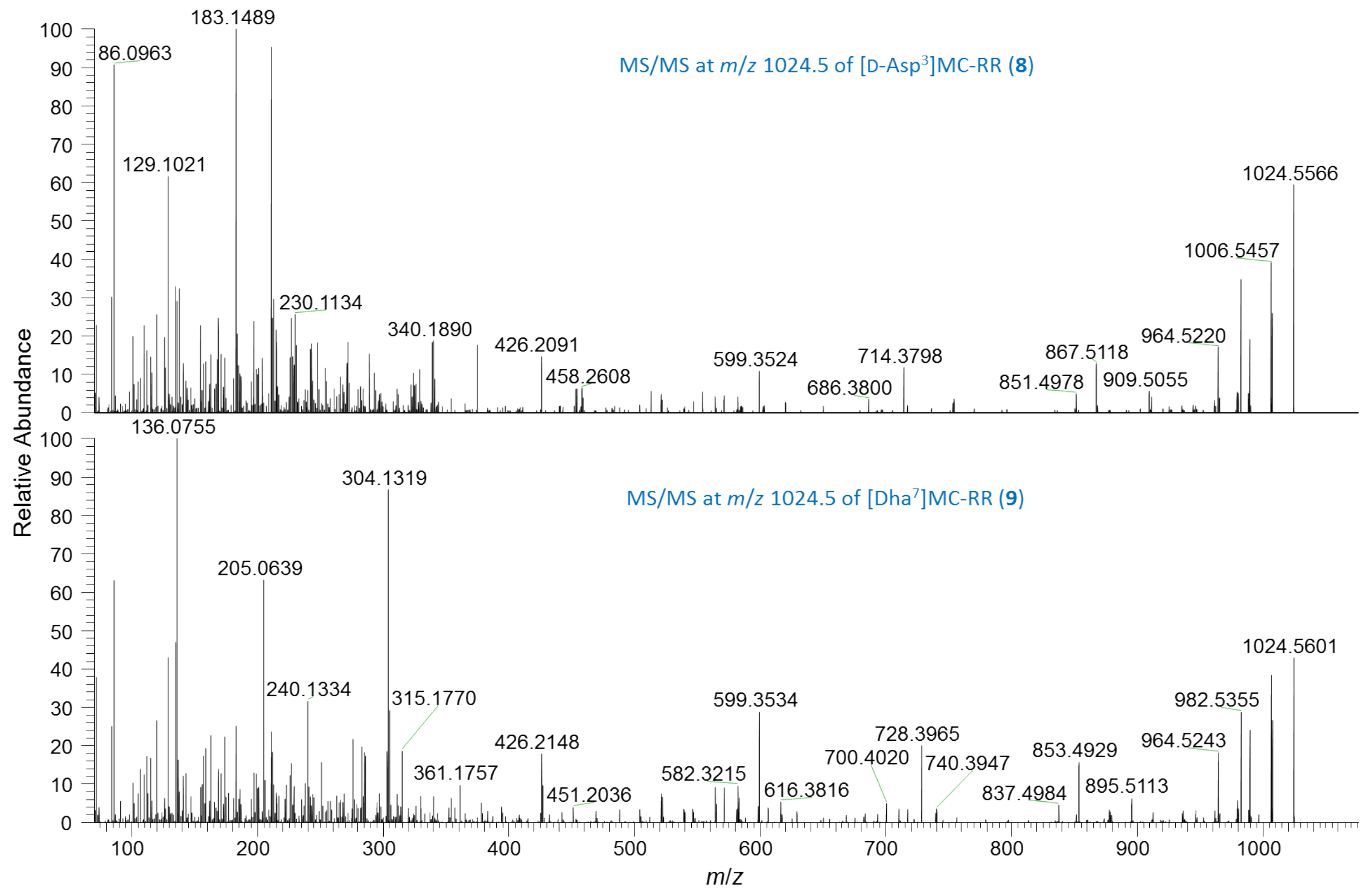

Figure S66. HRMS/MS spectra of $[\mathrm{M}+\mathrm{H}]^{+}$of $\left[\mathrm{Asp}^{3}\right] \mathrm{MC}-\mathrm{RR}(\mathbf{8})$ and [Dha $\left.{ }^{7}\right] \mathrm{MC}-\mathrm{RR}(\mathbf{9})$ from Figure S65. 


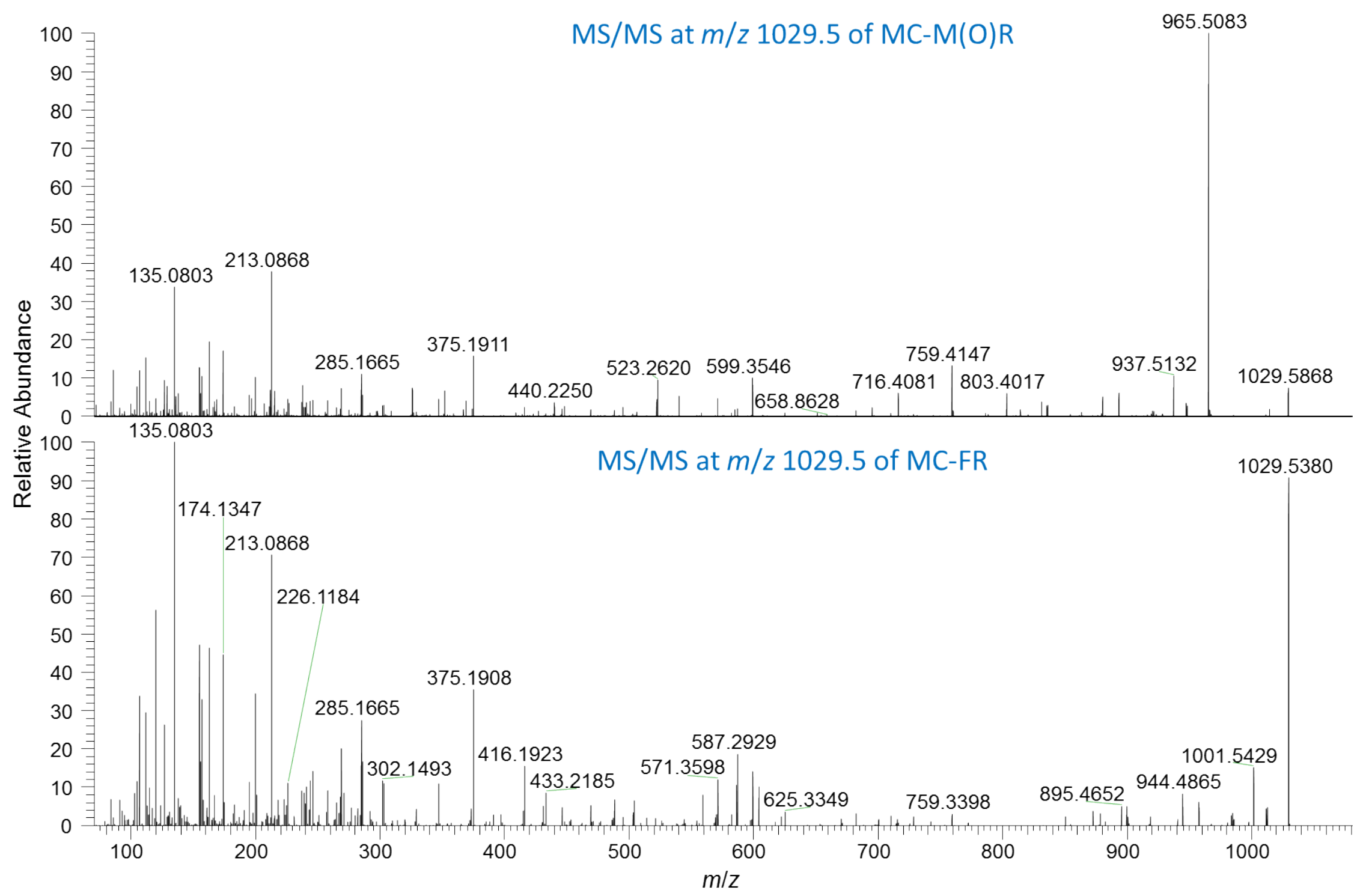

Figure S67. HRMS/MS spectra of $[\mathrm{M}+\mathrm{H}]^{+}$of MC-M(O)R (27) and MC-FR (28). Note the prominent neutral loss of $m / z 63.9983$ $\left(\mathrm{CH}_{3} \mathrm{SOH}\right)$ from the methionine $S$-oxide in $\mathrm{MC}-\mathrm{M}(\mathrm{O}) \mathrm{R}$. 


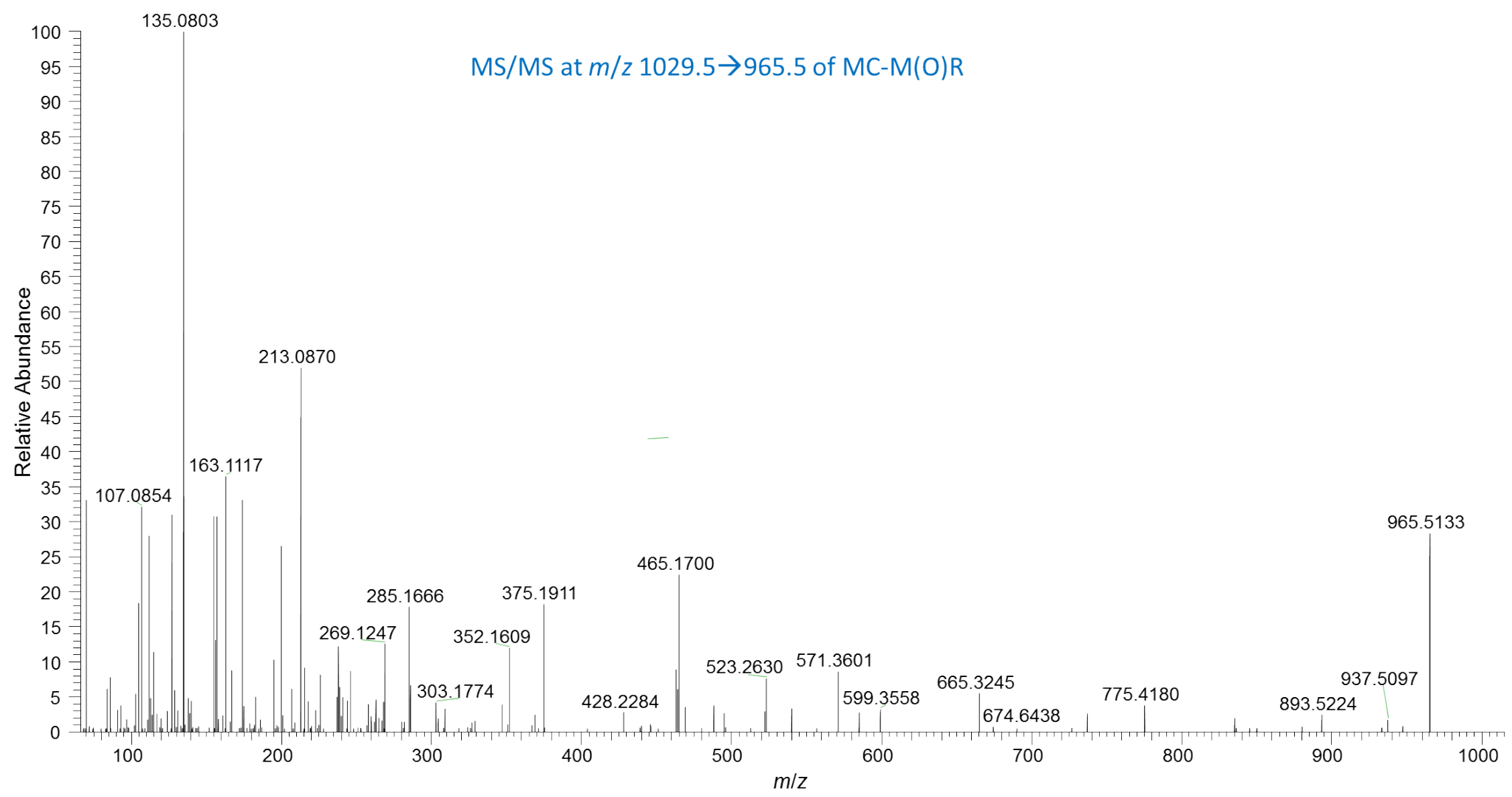

Figure S68. HRMS/MS spectrum of the $\left[\mathrm{M}+\mathrm{H}-\mathrm{CH}_{4} \mathrm{SO}\right]^{+}$product ion (at $\mathrm{m} / \mathrm{z} 965.5$, see Figure S67) produced by in-source fragmentation of MC-M(O)R (27). 


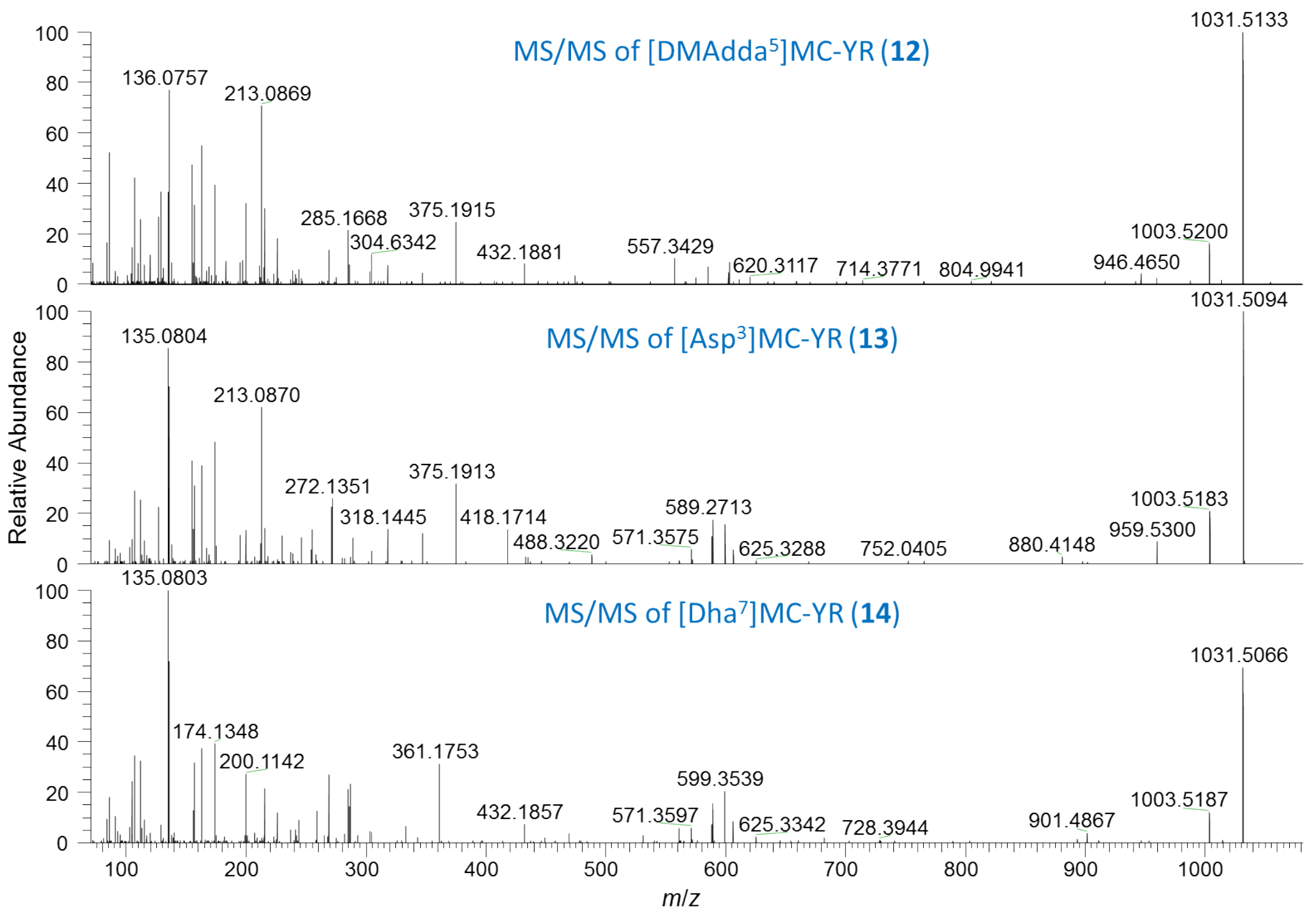

Figure S69. HRMS/MS spectra for $[\mathrm{M}+\mathrm{H}]^{+}$of $\left[\mathrm{DMAdda}{ }^{5}\right] \mathrm{MC}-\mathrm{YR}(\mathbf{1 2}),\left[\mathrm{Asp}^{3}\right] \mathrm{MC}-\mathrm{YR}(\mathbf{1 3})$, and $\left[\mathrm{Dha}^{7}\right] \mathrm{MC}-\mathrm{YR}(\mathbf{1 4})$. 


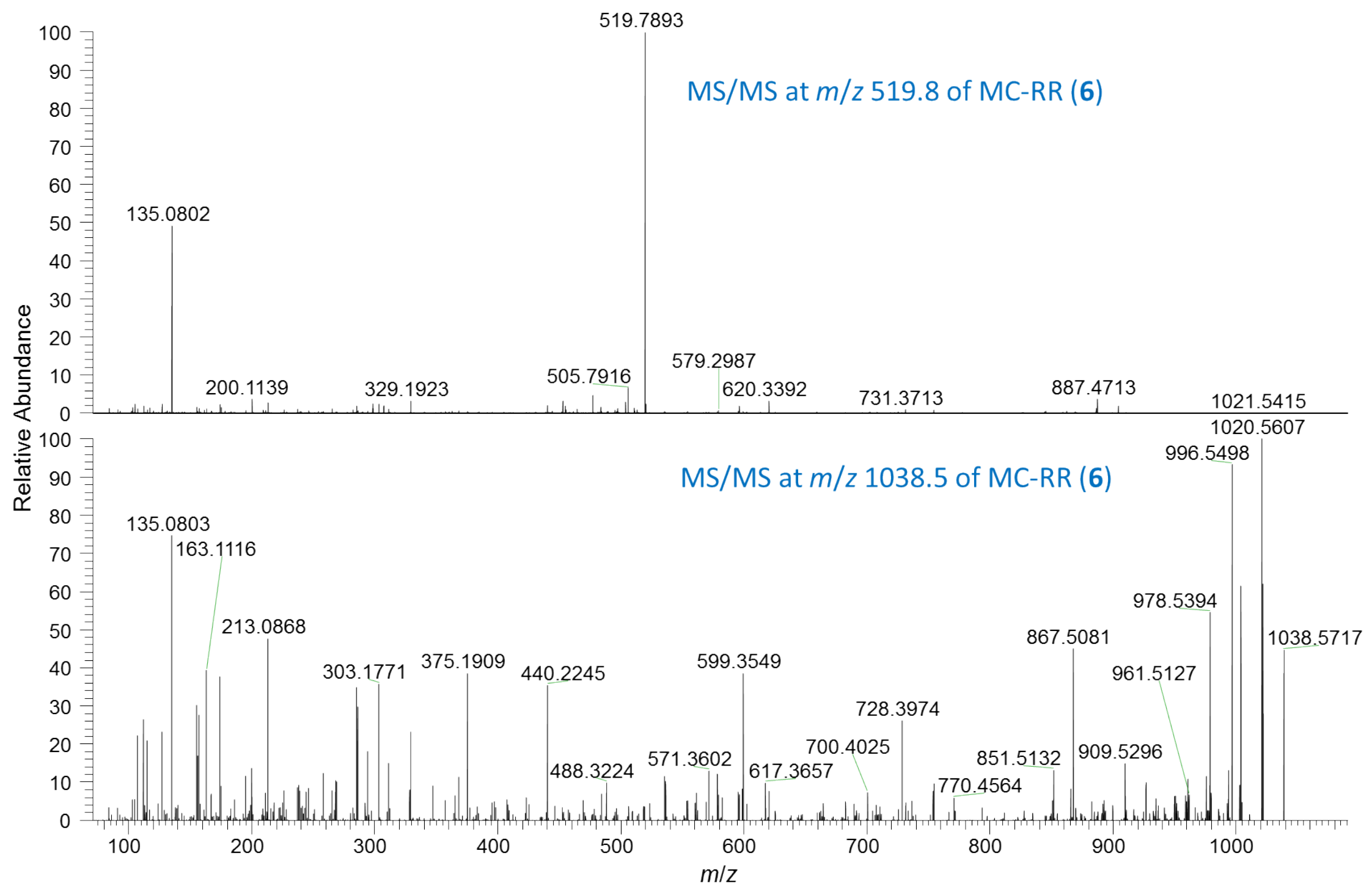

Figure S70. HRMS/MS spectra of: top, $[\mathrm{M}+2 \mathrm{H}]^{2+}$ at $\mathrm{m} / z$ 519.8, and; bottom, $[\mathrm{M}+\mathrm{H}]^{+} \mathrm{m} / z$ 1038.5, of MC-RR (6). 


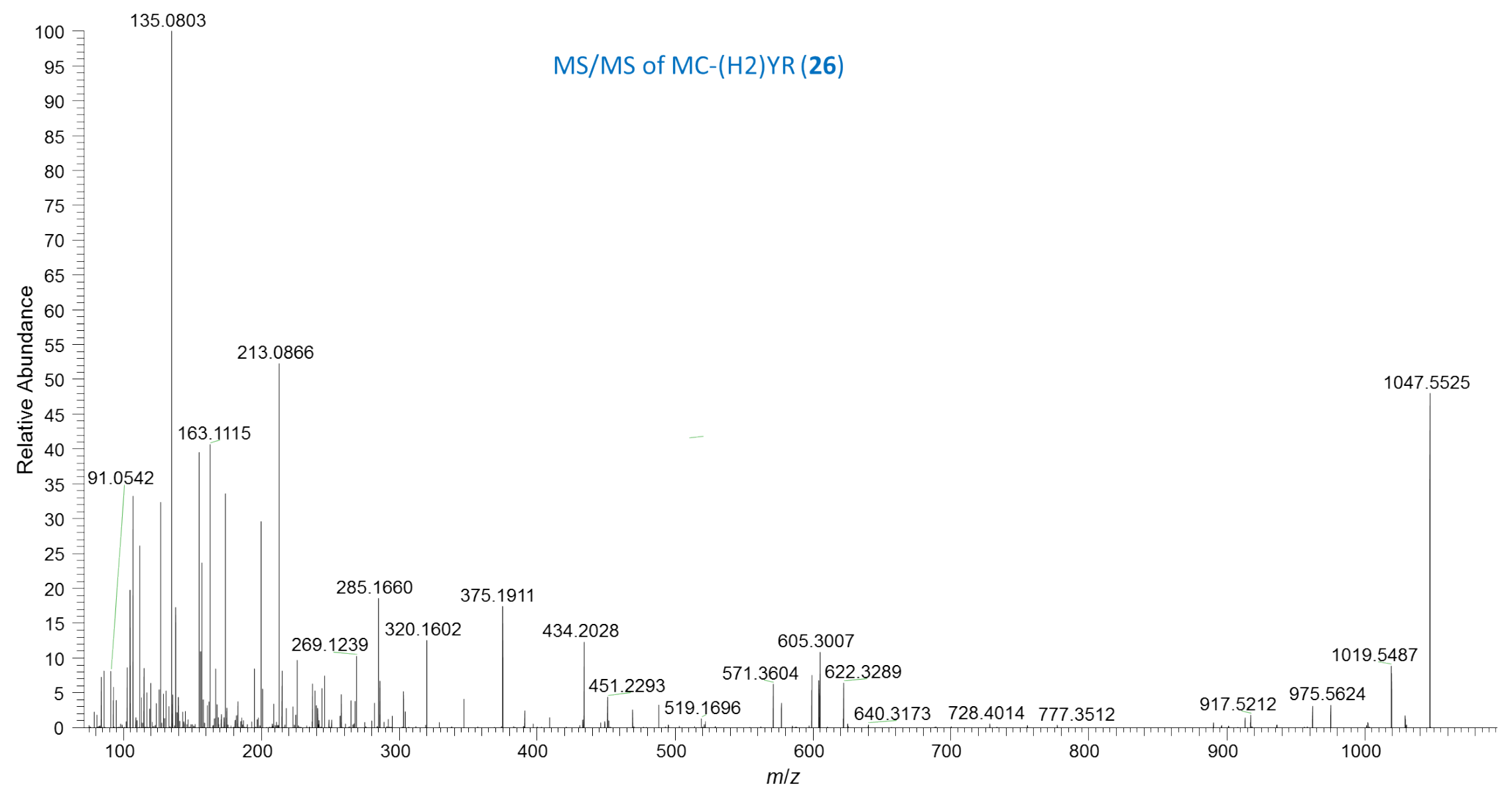

Figure S71. HRMS/MS spectrum of $[\mathrm{M}+\mathrm{H}]^{+}$of $\mathrm{MC}-(\mathrm{H} 2) \mathrm{YR}(\mathbf{2 6})$. 


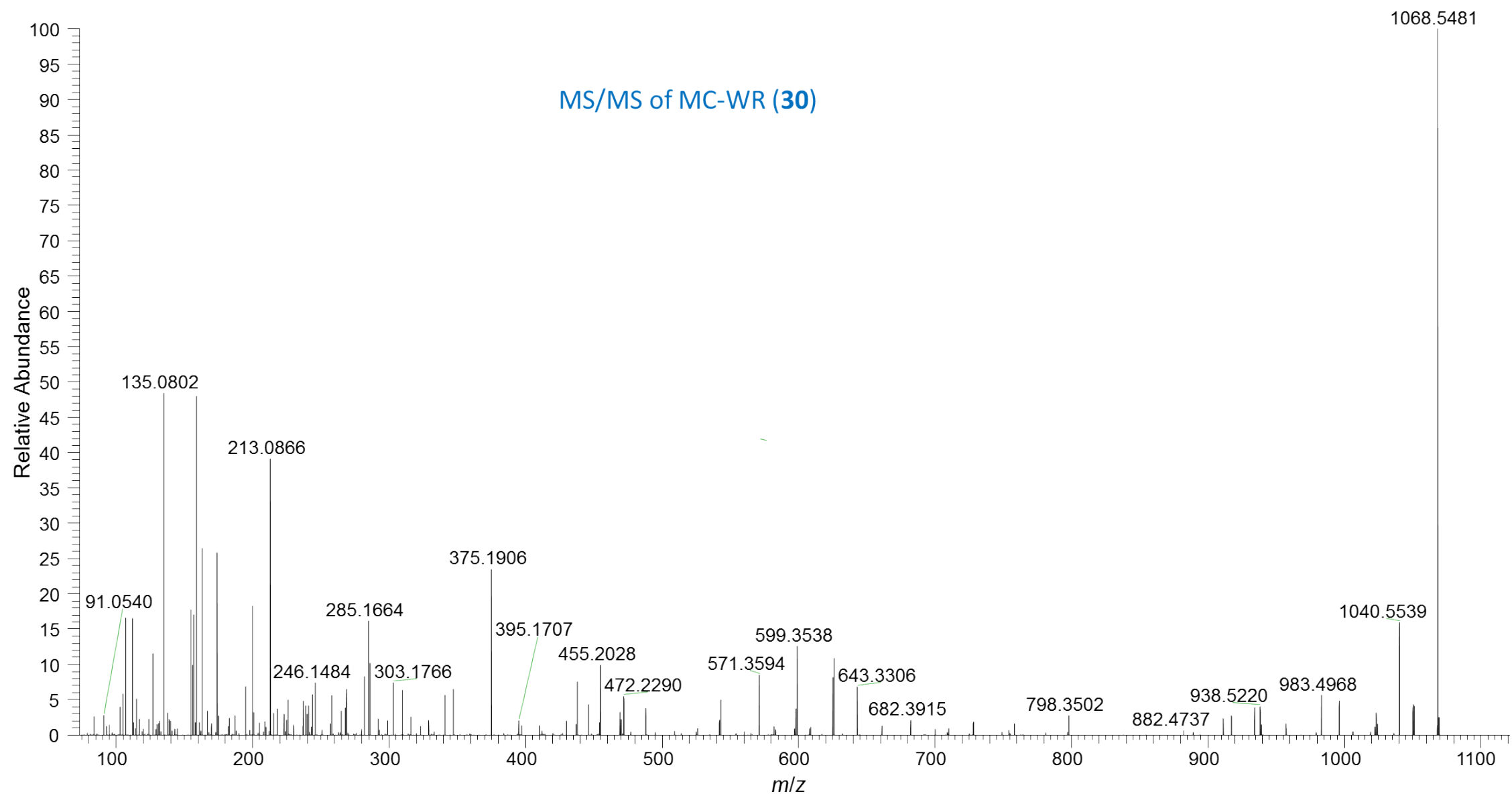

Figure S72. HRMS/MS spectrum of $[\mathrm{M}+\mathrm{H}]^{+}$of $\mathrm{MC}-\mathrm{WR}(\mathbf{3 0})$. 


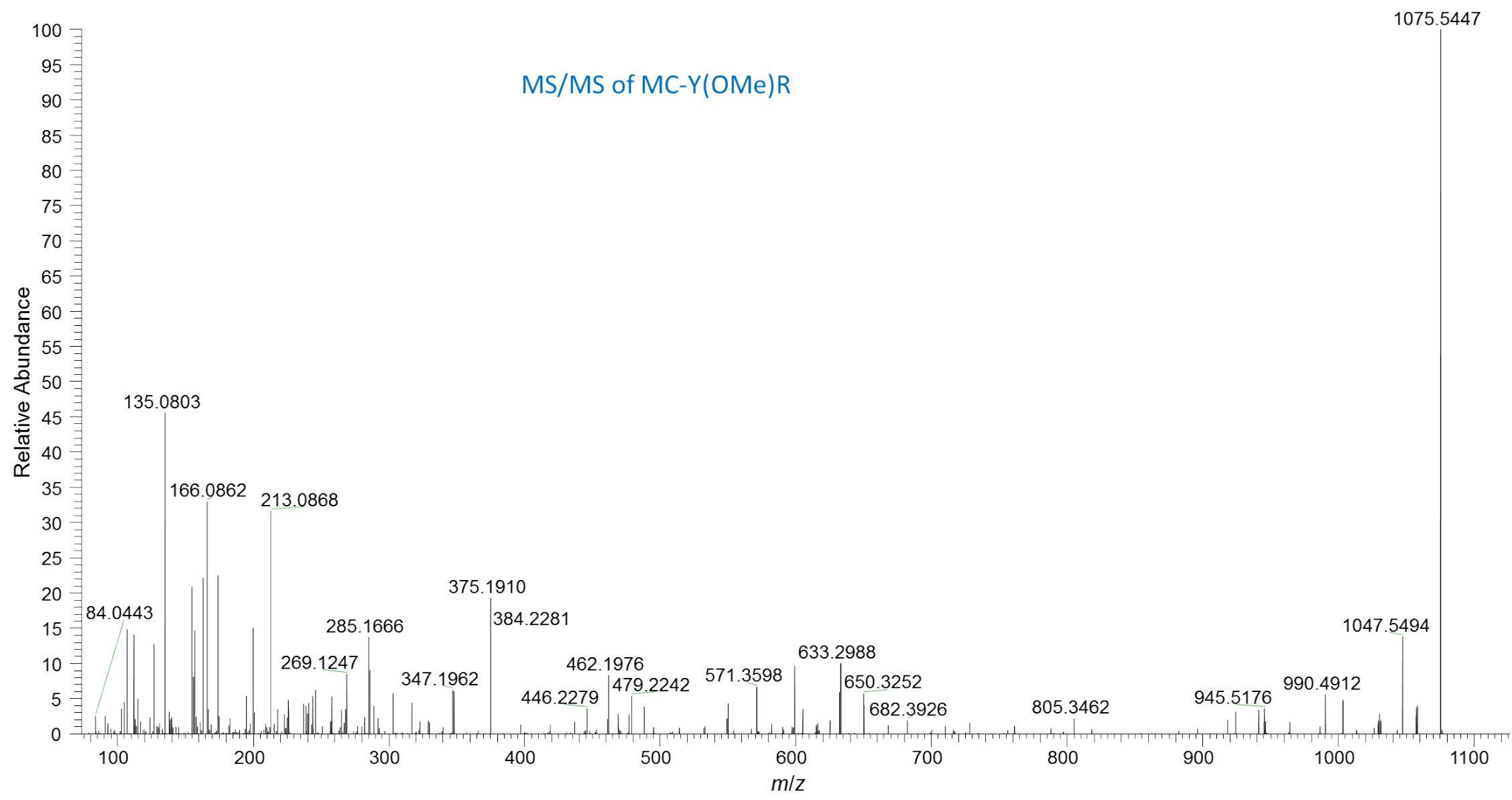

Figure S73. HRMS/MS spectrum of $[\mathrm{M}+\mathrm{H}]^{+}$of $\mathrm{MC}-\mathrm{Y}(\mathrm{OMe}) \mathrm{R}(\mathbf{3 1})$. 
Table S4. Observed $\mathrm{m} / \mathrm{z}$ shifts for identified product ions from $[\mathrm{M}+\mathrm{H}]^{+}$of MC-LA (16), MCLAbu (41), MC-LV (42), MC-(H4)Y(H4)Y (43), and [D-Ser ${ }^{1}$ MC-LA (44)

\begin{tabular}{|c|c|c|c|c|c|c|c|c|c|c|c|c|}
\hline $\mathrm{AA}$ Origin & \multicolumn{2}{|c|}{ Reported for MC-LA (16) } & \multicolumn{2}{|c|}{ MC-LA (16) } & \multicolumn{2}{|c|}{ MC-LAbu (41) } & \multicolumn{2}{|c|}{ MC-LV (42) } & \multicolumn{2}{|c|}{ MC-(H4)Y(H4)Y (43) } & \multicolumn{2}{|c|}{$\left[\mathrm{Ser}^{1}\right] \mathrm{MC}-\mathrm{LA}(\mathbf{4 4 )}$} \\
\hline \begin{tabular}{l|l}
6 & 7
\end{tabular} & Calc. $m / z$ & Formula & $\mathrm{m} / \mathrm{z}$ & Diff. & $\mathrm{m} / \mathrm{z}$ & Diff. & $m / z$ & Diff. & $m / z$ & Diff. & $m / z$ & Diff. \\
\hline & 64 & $\mathrm{C}_{5} \mathrm{H}_{12} \mathrm{~N}$ & 5.0964 & 000 & 86.0962 & 0002 & - & E & 122.09 & 5.9999 & - & - \\
\hline & 855 & $\mathrm{I}_{11}^{+}$ & .0854 & -0.0001 & 107.0854 & .0001 & - & - & - & - & - & - \\
\hline & & & 5.0803 & -0.0001 & 5.0803 & 0001 & 5.08 & 00 & 35.0 & .0 & 5.0 & 0.0 \\
\hline & & $\mathrm{C}_{10} \mathrm{H}_{15}{ }^{+}$ & 5.1166 & -0.0002 & 135.1166 & 0002 & - & - & - & - & - & - \\
\hline & 815 & $\mathrm{H}_{11} \mathrm{~N}_{2}$ & 5.0813 & -0.0002 & 155.0814 & .0001 & - & - & - & - & - & - \\
\hline & 163.1117 & & 3.1115 & -0.0002 & 163.1116 & 0.0001 & 3.1117 & 0.0000 & 63.1 & 0.0001 & 3.11 & 0.00 \\
\hline & 173 & $\mathrm{H}_{13}$ & 3.0918 & -0.0003 & 187.1075 & .0154 & 1.1235 & 28.0314 & 51.13 & 8.0466 & 173.0919 & -0.00 \\
\hline & & $\mathrm{C}_{9} \mathrm{H}_{11} \mathrm{~N}$ & 5.0763 & -0.0001 & 195.0763 & -0.0001 & 5.0763 & -0.0001 & 195.07 & -0.0001 & 195.07 & 0.00 \\
\hline & 213.0870 & & 3.0868 & -0.0002 & 213.0867 & -0.0003 & 13.0869 & -0.0001 & 213.0867 & -0.0003 & 213.0867 & -0.00 \\
\hline & 218.1135 & $\mathrm{H}_{16} \mathrm{~N}$ & 18.1133 & -0.0002 & 232.1289 & 14.0154 & 246.1453 & 28.0318 & 296.1601 & 78.0466 & 218.1135 & 0.0000 \\
\hline & & ${ }_{17} \mathrm{H}_{24}$ & 49 & -0.0003 & 258.1848 & 04 & 50 & -0.0 & 2. & 005 & & 0.0 \\
\hline & & & 81 & -0.0006 & 265.1583 & -0.0004 & 265.1590 & 0.0003 & 265.1580 & -0.0007 & 265.1583 & -0.00 \\
\hline & 268 & ( & 8.1650 & -0.0006 & 268.1651 & -0.0005 & 268.1654 & -0.0002 & 304.1651 & 35.9995 & 284.1601 & 15.9945 \\
\hline & 543 & $\mathrm{C}_{16} \mathrm{H}_{22} \mathrm{NO}_{4}$ & 2.1538 & -0.0005 & 292.1539 & -0.0004 & 292.1549 & 0.0006 & 292.152 & -0.0014 & 292.1530 & -0.0013 \\
\hline & & $\mathrm{C}_{20} \mathrm{H}_{2}$ & 44 & 0005 & 297.1845 & 04 & 97.1846 & 03 & 29 & 06 & 29 & -0.00 \\
\hline & 10 & & 4.1705 & 5 & 328 & 51 & 11 & 28 & 71 & 61 & 3 & $\overline{0.00}$ \\
\hline & 331.1976 & $\mathrm{C}_{14} \mathrm{H}_{2}$ & 331.1970 & -0.0006 & 345.2127 & 14.0151 & 359.2286 & 28.0310 & 445.2441 & 114.0465 & 331.1975 & -0.0001 \\
\hline & 347.1965 & $\mathrm{C}_{19} \mathrm{H}_{2}$ & 347.1960 & -0.0005 & 347.1956 & 0.0009 & 347.1956 & \begin{tabular}{|l|}
-0.0009 \\
\end{tabular} & 347.1954 & -0.0011 & 347.1960 & -0.000 \\
\hline & & & 5.1906 & - & 0 & 04 & 11 & -( & 3 & -0 & 37 & -0. \\
\hline & 82 & & 385.2073 & \begin{tabular}{|l|}
-0.0009 \\
\end{tabular} & 399. & 148 & 2394 & 28. & 538 & 114.0456 & 21 & 15. \\
\hline & 397.2082 & $\mathrm{C}_{18} \mathrm{H}_{2}$ & 397.2079 & \begin{tabular}{|l|}
-0.0003 \\
\end{tabular} & 397.2076 & -0.0006 & 397.2078 & -0.0004 & 433.2068 & 35.9986 & 413.2021 & 15.993 \\
\hline & 402.2347 & $\mathrm{C}_{17} \mathrm{H}_{3}$ & 402.2342 & -0.0005 & \begin{tabular}{|l|}
416.2495 \\
\end{tabular} & 14.0148 & 430.2660 & 28.0313 & 516.2806 & 114.0459 & 418.2291 & 15.9944 \\
\hline & 86 & & 46.2276 & - & 75 & 11 & 30 & -1 & 75 & 11 & 32 & 15.9946 \\
\hline & 453 & $\mathrm{C}_{21} \mathrm{H}_{3}$ & 443 & -0.0010 & 482.2602 & 0149 & .2762 & 09 & 15 & 14.0462 & 97 & 5.99 \\
\hline & 485.2718 & $\mathrm{C}_{21} \mathrm{H}_{3}$ & 485.2708 & -0.0010 & 499.2863 & 14.0145 & 513.3025 & 28.0307 & 599.3179 & 114.0461 & 501.2663 & 15.9945 \\
\hline & 509.2646 & & 509.2637 & \begin{tabular}{|l|}
-0.0009 \\
\end{tabular} & 509.2636 & -0.0010 & 509.2640 & -0.0006 & 509.2644 & -0.0002 & 509.2643 & -0.0003 \\
\hline & & & 17 & -0. & 55 & 07 & 14 & & & 985 & 65 & 15.9939 \\
\hline & 17 & $\mathrm{C}_{32} \mathrm{H}_{42} \mathrm{~N}_{3} \mathrm{O}_{7}^{+}$ & 08 & \begin{tabular}{|l|}
-0.0009 \\
\end{tabular} & 580.3012 & -0.0005 & 580.3007 & -0 & 5 & 0.0012 & 947 & 15.99 \\
\hline & 879 & $\mathrm{C}_{26} \mathrm{H}_{4}$ & 597.2872 & -0.0007 & 611.3032 & 14.0153 & 625.3191 & 28.0312 & 711.3310 & 114.0431 & 613.2824 & 15.9945 \\
\hline & 693.3858 & $\mathrm{C}_{38} \mathrm{H}_{53}$ & 693.3854 & -0.0004 & 693.3841 & -0.0017 & 693.3872 & 0.0014 & 729.3847 & 35.9989 & 709.3794 & 15.9936 \\
\hline & 4083 & $\mathrm{C}_{37} \mathrm{H}_{5}$ & 758.4076 & -0.0007 & 772.4204 & 14.0121 & 6.4381 & 28.0298 & 537 & 14.0454 & 774.4012 & 5.99 \\
\hline & 759.3923 & $\mathrm{C}_{37} \mathrm{H}_{55} \mathrm{~N}_{6} \mathrm{O}_{11}{ }^{+}$ & 759.3917 & -0.0006 & 773.4061 & 14.0138 & 787.4227 & 28.0304 & 391 & 14.0468 & 775.3847 & 15.99 \\
\hline & 776.4189 & $\mathrm{C}_{37} \mathrm{H}_{58} \mathrm{~N}_{7} \mathrm{O}_{11}{ }^{+}$ & 776.4187 & -0.0002 & 790.4326 & 14.0137 & 804.4496 & 28.0307 & 890.4660 & 114.0471 & 792.4122 & 15.9933 \\
\hline & 910.4920 & & 910.4929 & 0.0009 & 924.5078 & 14.0158 & 938.5241 & 28.0321 & 1060.5599 & 150.0679 & 926.4871 & 15.9951 \\
\hline Exact $m / z$ & 910.4920 & $\mathrm{C}_{46} \mathrm{H}_{68}$ & 910.4929 & 0.0009 & 924.5077 & 14.0157 & 938.5233 & 28.0313 & 1060.5601 & 150.0681 & 926.4870 & 15.995 \\
\hline
\end{tabular}


Table S5. Observed $\mathrm{m} / \mathrm{z}$ shifts for identified product ions from $[\mathrm{M}+\mathrm{H}]^{+}$of MC-LR (1), [DSer $^{1}$ ]MC-LR (34) and [D-Abu ${ }^{1}$ MC-LR (32)

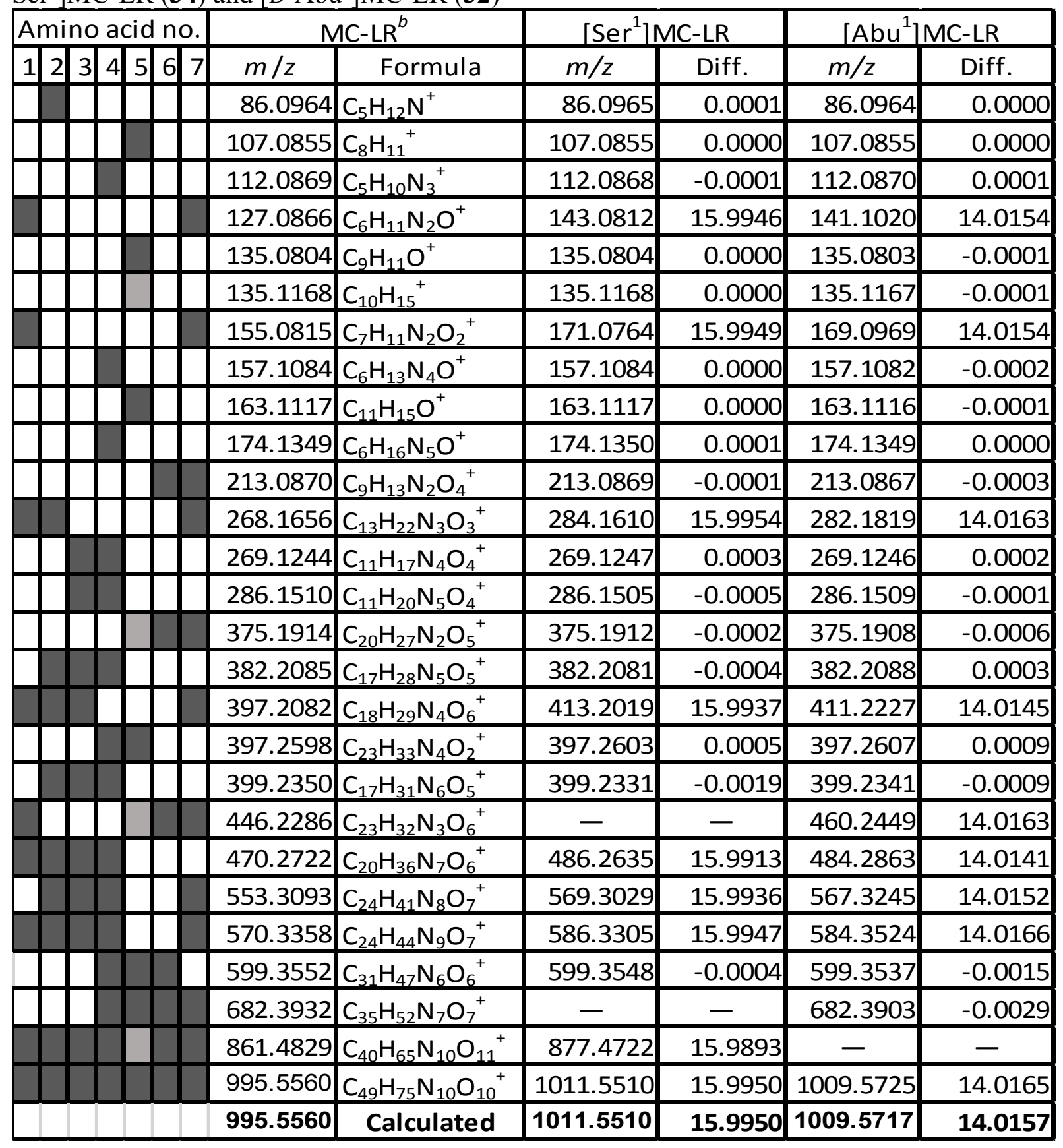


Recall notices for supplements TM07 and TM11 can be found at the following links:

TM07: https://www.accessdata.fda.gov/scripts/ires/index.cfm?Product=166417

TM1 1: $\underline{\text { https://www.accessdata.fda.gov/scripts/ires/index.cfm?Product=166097 }}$ 
Table S6. Estimation of total microcystin recalled.

\begin{tabular}{|l|r|l|l|l|r|r|r|}
\hline Supplement & $\begin{array}{l}\text { Bottles } \\
\text { Recalled }\end{array}$ & $\begin{array}{l}\text { Capsules } \\
\text { per } \\
\text { Bottle }\end{array}$ & $\begin{array}{l}\text { Grams } \\
\text { Product/Capsule }\end{array}$ & $\begin{array}{l}\text { aMC } \\
(\boldsymbol{\mu g} / \mathbf{g})\end{array}$ & $\begin{array}{l}\text { MC } \\
(\boldsymbol{\mu g} / \mathbf{c a p s u l e})\end{array}$ & $\begin{array}{l}\text { MC } \\
(\boldsymbol{\mu g} / \mathbf{b o t t l e})\end{array}$ & $\begin{array}{l}\text { Total MC } \\
\text { recalled } \\
(\boldsymbol{\mu g})\end{array}$ \\
\hline TM07 & 71,409 & 120 & 0.5 & 60 & 30 & 3600 & $\mathbf{2 5 7 , 0 7 2 , 4 0 0}$ \\
\hline TM11 & 4,226 & 90 & 0.3 & 40 & 12 & 1080 & $\mathbf{4 , 5 6 4 , 0 8 0}$ \\
\hline
\end{tabular}

